\title{
NONCOMMUTATIVE DUNKL OPERATORS AND BRAIDED CHEREDNIK ALGEBRAS
}

\author{
YURI BAZLOV AND ARKADY BERENSTEIN
}

\begin{abstract}
We introduce braided Dunkl operators $\underline{\nabla}_{1}, \ldots, \underline{\nabla}_{n}$ that are acting on a $\mathbf{q}$-symmetric algebra $S_{\mathbf{q}}\left(\mathbb{C}^{n}\right)$ and $\mathbf{q}$-commute. Generalising the approach of Etingof and Ginzburg, we explain the $\mathbf{q}$-commutation phenomenon by constructing braided Cherednik algebras $\underline{\mathcal{H}}$ for which the above operators form a representation. We classify all braided Cherednik algebras using the theory of braided doubles developed in our previous paper. Besides ordinary rational Cherednik algebras, our classification gives new algebras $\underline{\mathcal{H}}\left(W_{+}\right)$attached to an infinite family of subgroups of even elements in complex reflection groups, so that the corresponding braided Dunkl operators $\underline{\nabla}_{i}$ pairwise anti-commute. We explicitly compute these new operators in terms of braided partial derivatives and $W_{+}$-divided differences.
\end{abstract}

\section{Contents}

0. Introduction

1. Quadratic doubles

2. q-Cherednik algebras

3. Braided Cherednik algebras

4. Classification of braided Cherednik algebras

5. Universal embeddings and braided Dunkl operators References

\section{INTRODUCTION}

In 1989, Dunkl introduced the remarkable operators $\nabla_{1}, \ldots, \nabla_{n}$ that act on the polynomial algebra $\mathbb{C}\left[x_{1}, \ldots, x_{n}\right]$ by

$$
\nabla_{i}=\frac{\partial}{\partial x_{i}}+c \sum_{j \neq i} \frac{1}{x_{i}-x_{j}}\left(1-s_{i j}\right),
$$

where $s_{i j}$ is the automorphism of $\mathbb{C}\left[x_{1}, \ldots, x_{n}\right]$ switching the variables $x_{i}$ and $x_{j}$; together, the $s_{i j}$ generate the symmetric group $\mathbb{S}_{n}$. Dunkl operators are a very special deformation (with parameter

2000 Mathematics Subject Classification. Primary 20G42; Secondary 16S80, 20 F55.

The authors acknowledge support of the EPSRC grant EP/D065801/1 (Y.B.) and NSF grant DMS-0501103 (A.B.). 
$c \in \mathbb{C}$ ) of the partial derivatives $\frac{\partial}{\partial x_{i}}$, because they commute: $\nabla_{i} \nabla_{j}=\nabla_{j} \nabla_{i}$ for all $i, j$. This striking fact, originally proved in D Dy a lengthy direct computation, is interpreted in quantum mechanical terms as the integrability of the Calogero-Moser system, and algebraically via rational Cherednik algebras introduced in 2002 by Etingof and Ginzburg [EG]. A family of commuting Dunkl operators is attached not only to $\mathbb{S}_{n}$ but to any finite group generated by (complex) reflections.

The motivating idea behind the present paper is to introduce analogues of Dunkl operators on noncommutative deformations of the symmetric algebra $S(V)$, e.g., on the q-symmetric algebra

$$
\left.S_{\mathbf{q}}(V)=\mathbb{C}\left\langle x_{1}, \ldots, x_{n}\right| x_{i} x_{j}=q_{i j} x_{j} x_{i} \text { for } i<j\right\rangle
$$

where $V$ is a $\mathbb{C}$-vector space with basis $x_{1}, \ldots, x_{n}$ and $\mathbf{q}=\left(q_{i j}\right)$ is a complex $n \times n$ matrix.

Assume that $q_{i j} q_{j i}=q_{i i}=1$ for all $i, j$ and define braided partial derivatives $\underline{\partial}_{i}: S_{\mathbf{q}}(V) \rightarrow S_{\mathbf{q}}(V)$ by

$$
\underline{\partial}_{i}\left(x_{1}^{a_{1}} \cdots x_{i}^{a_{i}} \cdots x_{n}^{a_{n}}\right)=a_{i} q_{1, i}^{a_{1}} \cdots q_{i-1, i}^{a_{i-1}} x_{1}^{a_{1}} \cdots x_{i}^{a_{i}-1} \cdots x_{n}^{a_{n}} .
$$

Clearly, $\underline{\partial}_{i} \underline{\partial}_{j}=q_{i j} \underline{\partial}_{j} \underline{\partial}_{i}$ and $\underline{\partial}_{i} x_{j}=q_{j i} \underline{\partial}_{i} x_{j}+\delta_{i j}$ for all $i, j$.

It turns out that if $q_{i j}=-1$ for all $i \neq j$ (denote such matrix $\mathbf{q}$ by $\mathbf{- 1}$ ), then we can introduce the following deformations $\underline{\nabla}_{i}: S_{-\mathbf{1}}(V) \rightarrow S_{-\mathbf{1}}(V)$ of the braided partial derivatives $\underline{\partial}_{i}$. Let $\mathcal{C}^{\prime} \subset \mathcal{C}$ be finite (cyclic) subgroups of $\mathbb{C}^{\times}$such that $-1 \in \mathcal{C}$ (i.e., $\mathcal{C}$ is of even order), and let $c: \mathcal{C}^{\prime} \rightarrow \mathbb{C}$ be a function $\varepsilon^{\prime} \mapsto c_{\varepsilon^{\prime}}$. For each $i=1, \ldots, n$ define the operator $\underline{\nabla}_{i}$ on the skew-field of fractions of $S_{-1}(V)$ as follows:

$$
\underline{\nabla}_{i}=\underline{\partial}_{i}+c_{1} \sum_{j \neq i, \varepsilon \in \mathcal{C}} \frac{x_{i}+\varepsilon x_{j}}{x_{i}^{2}-\varepsilon^{2} x_{j}^{2}}\left(1-\sigma_{i j}^{(\varepsilon)}\right)+\sum_{\varepsilon^{\prime} \in \mathcal{C}^{\prime} \backslash\{1\}} \frac{c_{\varepsilon^{\prime}}}{1-\varepsilon^{\prime}} \cdot \frac{1}{x_{i}}\left(1-t_{i}^{\left(\varepsilon^{\prime}\right)}\right),
$$

where $t_{i}^{(\varepsilon)}, \sigma_{i j}^{(\varepsilon)}$ are algebra automorphisms of $S_{-\mathbf{1}}(V)$ defined by

$$
t_{i}^{(\varepsilon)}\left(x_{k}\right)=\left\{\begin{array}{ll}
x_{k} & \text { if } k \neq i \\
\varepsilon x_{i} & \text { if } k=i
\end{array} ; \quad \sigma_{i j}^{(\varepsilon)}\left(x_{k}\right)=\left\{\begin{array}{ll}
x_{k} & \text { if } k \notin\{i, j\} \\
\varepsilon x_{j} & \text { if } k=i \\
-\varepsilon^{-1} x_{i} & \text { if } k=j
\end{array} \quad \text { for all } i \neq j, \varepsilon \in \mathbb{C}^{\times} .\right.\right.
$$

We refer to these new operators $\underline{\nabla}_{i}$ as noncommutative or braided Dunkl operators due to the following

Theorem 0.1. The operators $\underline{\nabla}_{1}, \ldots, \underline{\nabla}_{n}$ preserve $S_{-\mathbf{1}}(V)$ and satisfy $\underline{\nabla}_{i} \underline{\nabla}_{j}+\underline{\nabla}_{j} \underline{\nabla}_{i}=0$ for all $i \neq j$.

Therefore, the operators $\underline{\nabla}_{i}$ may be viewed as an anti-commutative analogue of a Calogero-Moser integrable system. We will elaborate on the connection with integrable systems in a separate paper. 
We prove Theorem 0.1 by means of braided Cherednik algebras which we introduce (following the logic of [EG]) as the main tool for establishing anti- and $q$-commutation relations between operators (10) and their generalisations.

Namely, let $W_{\mathcal{C}, \mathcal{C}^{\prime}}$ be the subgroup of $\operatorname{GL}(V)$ generated by all $\sigma_{i j}^{(\varepsilon)}, \varepsilon \in \mathcal{C}$ and $t_{i}^{\left(\varepsilon^{\prime}\right)}, \varepsilon^{\prime} \in \mathcal{C}^{\prime}$. Define $\underline{\mathcal{H}}_{c}\left(W_{\mathcal{C}, \mathcal{C}^{\prime}}\right)$ to be the subalgebra of $\operatorname{End}_{\mathbb{C}}\left(S_{-\mathbf{1}}(V)\right)$ generated by $W_{\mathcal{C}, \mathcal{C}^{\prime}}, y_{i}=\underline{\nabla}_{i}$, and operators of multiplication by $x_{i}$.

Theorem 0.2. (a) In the algebra $\underline{\mathcal{H}}_{c}\left(W_{\mathcal{C}, \mathcal{C}^{\prime}}\right)$, the generators satisfy:

(i) $x_{i} x_{j}+x_{j} x_{i}=y_{i} y_{j}+y_{j} y_{i}=0$ for all $i \neq j$;

(ii) $w x_{i} w^{-1}=w\left(x_{i}\right), w y_{i} w^{-1}=w\left(y_{i}\right)$ for all $w \in W_{\mathcal{C}, \mathcal{C}^{\prime}}, i=1, \ldots, n$;

(iii) $y_{j} x_{i}+x_{i} y_{j}=c_{1} \sum_{\varepsilon \in \mathcal{C}} \varepsilon \sigma_{i j}^{(\varepsilon)}$ for all $i \neq j$, and $y_{i} x_{i}-x_{i} y_{i}=1+c_{1} \sum_{j \neq i, \varepsilon \in \mathcal{C}} \sigma_{i j}^{(\varepsilon)}+\sum_{\varepsilon^{\prime} \in \mathcal{C}^{\prime} \backslash\{1\}} c_{\varepsilon^{\prime}} t_{i}^{\left(\varepsilon^{\prime}\right)}$ for $i=1, \ldots, n$.

(b) As an associative algebra, $\underline{\mathcal{H}}_{c}\left(W_{\mathcal{C}, \mathcal{C}^{\prime}}\right)$ is determined by relations $(i)-($ iii $)$ and admits a triangular decomposition

$$
\underline{\mathcal{H}}_{c}\left(W_{\mathcal{C}, \mathcal{C}^{\prime}}\right)=S_{-\mathbf{1}}(V) \otimes \mathbb{C} W_{\mathcal{C}, \mathcal{C}^{\prime}} \otimes S_{-\mathbf{1}}\left(V^{*}\right) .
$$

We prove Theorem 0.2 in Section 3.3 In what follows we will refer to $\mathcal{H}_{c}\left(W_{\mathcal{C}, \mathcal{C}^{\prime}}\right)$ as a negative braided Cherednik algebra. Using the Verma module $M_{\text {triv }}=S_{-\mathbf{1}}(V)$ for $\underline{\mathcal{H}}_{c}\left(W_{\mathcal{C}, \mathcal{C}^{\prime}}\right)$, induced from the trivial representation of $W_{\mathcal{C}, \mathcal{C}^{\prime}}$, we obtain the following refinement of Theorem 0.1 (to be proved in Section 5.3.

Theorem 0.3. The generators $y_{i}$ of the negative braided Cherednik algebra $\underline{\mathcal{H}}_{c}\left(W_{\mathcal{C}, \mathcal{C}^{\prime}}\right)$ act on its Verma module $M_{\text {triv }}=S_{-\mathbf{1}}(V)$ by braided Dunkl operators (1).

Remark 0.4. In fact, if one drops the constant term 1 in the second relation of Theorem $0.2(a)(i i i)$ and at the same time drops the braided derivative $\underline{\partial}_{i}$ in (1), then one obtains a degenerate braided Cherednik algebra for which Theorems 0.2 and 0.3 are also valid. The latter algebra admits a finitedimensional quotient by the $W_{\mathcal{C}, \mathcal{C}^{\prime}}$-invariant ideals of $S_{\mathbf{q}}(V)$ and $S_{\mathbf{q}}\left(V^{*}\right)$, which is an analogue of restricted Cherednik algebras; see [G].

Remark 0.5. In a series of papers [KW1, KW2] Ta Khongsap and Weiqiang Wang have discovered a different class of algebras with triangular decomposition and anti-commuting generators. This similarity inspired us to start a new project [BB3] where constructions of [KW1, KW2 are uniformly treated in the context of braided doubles (developed in $[\mathrm{BB}$ and in Section 1 of the present paper).

The groups $W_{\mathcal{C}, \mathcal{C}^{\prime}}$ are classified in terms of the infinite family of classical complex reflection groups $G(m, p, n)=\mathbb{S}_{n} \ltimes T(m, p, n), m \geq 1, p \mid m$, where $\mathbb{S}_{n} \subset \mathrm{GL}(V)$ is the group of permutation 
matrices and $T(m, p, n)=\left\{\operatorname{diag}\left(\varepsilon_{1}, \ldots, \varepsilon_{n}\right) \mid \varepsilon_{i}^{m}=1 \forall i,\left(\varepsilon_{1} \ldots \varepsilon_{n}\right)^{m / p}=1\right\}$. It is not difficult to show that

- if $\left|\mathcal{C}^{\prime}\right|$ is even, $W_{\mathcal{C}, \mathcal{C}^{\prime}}=G(m, p, n)$ with $m=|\mathcal{C}|, p=\left|\mathcal{C} / \mathcal{C}^{\prime}\right|$;

— if $\left|\mathcal{C}^{\prime}\right|$ is odd, $W_{\mathcal{C}, \mathcal{C}^{\prime}}=G(m, p, n)_{+}$, a subgroup of index 2 in $G(m, p, n)$ with $m=|\mathcal{C}|$, $p=\left|\mathcal{C} / \pm \mathcal{C}^{\prime}\right|$, consisting of $g$ such that $\operatorname{det}(g)^{\left|\mathcal{C}^{\prime}\right|}=1$.

(Note however, that the generators $\sigma_{i j}^{(\varepsilon)}$ of $W_{\mathcal{C}, \mathcal{C}^{\prime}}$ are of order 4 and are not complex reflections.) The smallest example of such group in rank $n$ is $W_{\{ \pm 1\},\{1\}}=B_{n}^{+}$of even elements in a Coxeter group of type $B_{n}$; see Example 3.11

Having been inspired by the construction of the above negative braided Cherednik algebras corresponding to the matrix $\mathbf{q}=\mathbf{- 1}$, we formulated and solved the following problem.

Problem 0.6. Let $\mathbf{q}=\left(q_{i j}\right)$ be a complex $n \times n$ matrix with $q_{i j} q_{j i}=q_{i i}=1$ and $V$ be a vector space with basis $x_{1}, \ldots, x_{n}$. Find all finite groups $W \subset \mathrm{GL}(V)$ acting on $S_{\mathbf{q}}(V)$ by automorphisms and all algebras $\underline{\mathcal{H}}$ generated by $W, V$, and $V^{*}$ such that:

(a) $\underline{\mathcal{H}}$ admits a triangular decomposition $\underline{\mathcal{H}}=S_{\mathbf{q}}(V) \otimes \mathbb{C} W \otimes S_{\mathbf{q}}\left(V^{*}\right)$, where $S_{\mathbf{q}}(V) \otimes \mathbb{C} W$ and $\mathbb{C} W \otimes S_{\mathbf{q}}\left(V^{*}\right)$ are semidirect product algebras;

(b) $y_{j} x_{i}-q_{i j} x_{i} y_{j} \in \mathbb{C} W$ for all $i, j$, where $y_{1}, \ldots, y_{n}$ is the basis of $V^{*}$ dual to $x_{1}, \ldots, x_{n}$.

Clearly, ordinary rational Cherednik algebras $H_{c}(W)$ associated to complex reflection groups $W \subset \mathrm{GL}(V)$ and the above negative algebras $\underline{\mathcal{H}}_{c}\left(W_{\mathcal{C}, \mathcal{C}^{\prime}}\right)$ solve Problem 0.6 for special examples of the matrix q. We refer to solutions of Problem 0.6 as braided Cherednik algebras.

Let us briefly describe how to construct all braided Cherednik algebras out of the above building blocks (see Section 4 for details). Informally speaking, we prove that each braided Cherednik algebra is a braided product (which, once again, justifies the name) of rational Cherednik algebras and the negative algebras $\underline{\mathcal{H}}_{c}\left(W_{\mathcal{C}, \mathcal{C}^{\prime}}\right)$.

More precisely, let each of $\mathcal{H}\left(W_{1}\right), \ldots, \mathcal{H}\left(W_{m}\right)$ be either a rational Cherednik algebra of an irreducible complex reflection group $W_{k}$ (one of the groups $G(m, p, n)$ and $G_{4}, \ldots, G_{37}$ in ShephardTodd's classification [ST] ) or a negative braided Cherednik algebra of $W_{k}=W_{\mathcal{C}, \mathcal{C}^{\prime}}$. One has $\mathcal{H}\left(W_{k}\right) \cong S\left(V_{k}\right) \otimes \mathbb{C} W_{k} \otimes S\left(V_{k}^{*}\right)$ or $S_{-\mathbf{1}}\left(V^{k}\right) \otimes \mathbb{C} W_{k} \otimes S_{-\mathbf{1}}\left(V_{k}^{*}\right)$, respectively. Choose $m(m-1) / 2$ arbitrary parameters $r_{k l} \in \mathbb{C}^{\times}, 1 \leq k<l \leq m$. Define $\mathcal{H}$ to be the algebra generated by all $\mathcal{H}\left(W_{k}\right)$ subject to the relations

$$
x x^{\prime}=r_{k l} x^{\prime} x, \quad y y^{\prime}=r_{k l} y^{\prime} y, \quad x y^{\prime}=r_{k l}^{-1} y^{\prime} x, \quad y x^{\prime}=r_{k l}^{-1} x^{\prime} y
$$

for $x \in V_{k}, y \in V_{k}^{*}, x^{\prime} \in V_{l}, y^{\prime} \in V_{l}^{*}$, and the relation that $W_{k}$ commute with $\mathcal{H}\left(W_{l}\right)$ for $k \neq l$. In Section 5 , we prove 
Theorem 0.7. (a) $\mathcal{H}$ factorises as a tensor product of its subalgebras,

$$
\mathcal{H}=\mathcal{H}\left(W_{1}\right) \otimes \ldots \otimes \mathcal{H}\left(W_{m}\right)
$$

and is a braided Cherednik algebra of the group $W_{1} \times \cdots \times W_{m}$;

(b) each braided Cherednik algebra of a group $W$ is isomorphic to one of such algebras $\mathcal{H}$ (under a simple assumption of minimality of $W$, see Section 4.4.).

The braided Cherednik algebra $\mathcal{H}$ so constructed has factorisation $S_{\mathbf{q}}(V) \otimes \mathbb{C W} \otimes S_{\mathbf{q}}\left(V^{*}\right)$ with $V=V_{1} \oplus \cdots \oplus V_{m}$. Here $\mathbf{q}$ is the matrix consisting of $m^{2}$ blocks $M_{k l}$ (of size $\operatorname{dim} V_{k} \times \operatorname{dim} V_{l}$ ), $k, l=1, \ldots, m$. The block $M_{k k}$ has all off-diagonal entries equal to 1 (respectively -1 ) if $\mathcal{H}\left(W_{k}\right)$ is a rational (respectively negative braided) Cherednik algebra. The block $M_{k l}$ has all entries equal to $r_{k l}$ if $k<l$ or to $r_{l k}^{-1}$ if $k>l$.

The proof of the theorem is based on the theory of braided doubles developed in our previous paper [BB]. Results specific to quadratic braided doubles over group algebras are given in Section [1] Using these results, in Section 2 we introduce and classify q-Cherednik algebras as specific quadratic doubles with triangular factorisation $S_{\mathbf{q}}(V) \otimes \mathbb{C} \widetilde{W} \otimes S_{\mathbf{q}^{\top}}\left(V^{*}\right)$ where $\widetilde{W}$ is a certain Abelian extension of $W$ depending on $\mathbf{q}$ (and $\mathbf{q}^{\top}$ stands for the transposed matrix). Based on this classification and the braided reduction introduced in Section 1.7, we prove that each braided Cherednik algebra is naturally a subalgebra of one of the q-Cherednik algebras (Proposition 3.3), and, on the other hand, that each braided Cherednik algebra naturally admits a factorisation (2) in an appropriate braided tensor category (Theorem 4.8).

Let us illustrate our construction of braided Cherednik algebras and the corresponding braided Dunkl operators for any finite Abelian group $W$, i.e., $W=\mathcal{C}_{1} \times \mathcal{C}_{2} \times \cdots \times \mathcal{C}_{n}$, where each $\mathcal{C}_{i}$ is a finite (cyclic) subgroup of $\mathbb{C}^{\times}$, and an arbitrary $n \times n$ matrix $\mathbf{q}$ as above. Define the braided Dunkl operators $\underline{\nabla}_{1}, \ldots, \underline{\nabla}_{n}: S_{\mathbf{q}}(V) \rightarrow S_{\mathbf{q}}(V)$ attached to $W$ by

$$
\underline{\nabla}_{i}=\underline{\partial}_{i}+\sum_{\varepsilon \in \mathcal{C}_{i} \backslash\{1\}} \frac{c_{i, \varepsilon}}{1-\varepsilon} \cdot \frac{1}{x_{i}}\left(1-t_{i}^{(\varepsilon)}\right) .
$$

Proposition 0.8. The braided Dunkl operators $\underline{\nabla}_{1}, \ldots, \underline{\nabla}_{n}$ satisfy for all $i, j$ :

$$
\underline{\nabla}_{i} x_{j}-q_{j i} x_{j} \underline{\nabla}_{i}=\delta_{i j}\left(1+\sum_{\varepsilon \in \mathcal{C}_{i} \backslash\{1\}} c_{i, \varepsilon} t_{i}^{(\varepsilon)}\right), \quad \underline{\nabla}_{i} \underline{\nabla}_{j}=q_{i j} \underline{\nabla}_{j} \underline{\nabla}_{i} .
$$

In general, braided Dunkl operators attached to a direct product $W_{1} \times \cdots \times W_{m}$ of groups are obtained from Theorem 0.7 For each group $W_{k}$, one writes down either commuting Dunkl operators for complex reflection groups [DO] or anticommuting operators $\underline{\nabla}_{i}$ as above. The differential parts of these operators now become braided derivatives $\underline{\partial}_{i}$ of $S_{\mathbf{q}}(V)$, an altogether they form a complete list of braided Dunkl operators for $W$. 
Our next result deals with universal embedding of each braided Cherednik algebra in a modified Heisenberg double. This embedding is crucial in the proof of Theorem 0.1 and Theorem 0.3 Besides that, it leads to new interesting representations of braided Cherednik algebras (see below).

A modified Heisenberg double $\mathcal{A}$ is an algebra attached to two Yetter-Drinfeld module structures on the same module $Y$ over a group $W$. It has triangular decomposition $\mathcal{A}=U^{-} \otimes \mathbb{C} W \otimes U^{+}$, where

$$
U^{-}=T(Y) /\left\langle\wedge_{\Psi_{1}}^{2} Y \cap \wedge_{\Psi_{2}}^{2} Y\right\rangle, \quad U^{+}=T\left(Y^{*}\right) /\left\langle\wedge_{\Psi_{1}^{*}}^{2} Y^{*} \cap \wedge_{\Psi_{2}^{*}}^{2} Y^{*}\right\rangle,
$$

where $\wedge_{\Psi}^{2} X=\operatorname{ker}\left(\operatorname{id}_{X} \otimes X+\Psi\right)$ for $\Psi \in \operatorname{End}(X \otimes X)$ on any vector space $X$, and $\Psi_{1}, \Psi_{2}$ are braidings on $Y$ induced by the two Yetter-Drinfeld structures over $W$.

For a braided Cherednik algebra $\underline{\mathcal{H}}(W)=S_{\mathbf{q}}(V) \otimes \mathbb{C} W \otimes S_{\mathbf{q}}(V)$, there is an extension $\widetilde{W}=$ $W \cdot \Gamma \subset \operatorname{GL}(V)$ of $W$ by means of an Abelian group $\Gamma \subset \operatorname{GL}(V)$. To this data we associate a q-reflections module $Y$ over $\widetilde{W}$ with two Yetter-Drinfeld structures, hence a modified Heisenberg double $\mathcal{A}(\widetilde{W})=U^{-} \otimes \mathbb{C} \widetilde{W} \otimes U^{+}$.

Theorem 0.9. In the above setup, there exists an injective algebra homomorphism

$$
\varphi: \underline{\mathcal{H}}(W) \rightarrow \mathcal{A}(\widetilde{W})
$$

such that $\left.\varphi\right|_{W}$ is the natural inclusion of $W$ in $\widetilde{W}, \varphi(V) \subset Y$ and $\varphi\left(V^{*}\right) \subset \Gamma \cdot Y^{*}$.

The embedding $\varphi: \underline{\mathcal{H}}(W) \hookrightarrow \mathcal{A}(\widetilde{W})$ generalises our earlier result [BB, Theorem 7.26], where we constructed such embeddings for all rational Cherednik algebras. This way we can obtain new representations of $\underline{\mathcal{H}}(W)$ in $\mathcal{A}(\widetilde{W})$ or in the Verma-type $\mathcal{A}(\widetilde{W})$-module $U^{-}$.

The quadratic algebra $U^{-}$arising from Theorem 0.9 is itself of great interest. In [BK], Anatol Kirillov and the first author show that when $\underline{\mathcal{H}}(W)$ is a rational Cherednik algebra, the defining relations in $U^{-}$are generalised classical Yang-Baxter equations. In particular, if $W=\mathbb{S}_{n}, U^{-}$coincides with the triangular enveloping algebra $U\left(\operatorname{tr}_{n}\right)$ of Bartholdi-Enriquez-Etingof-Rains [BEER; moreover, $U\left(\operatorname{tr}_{n}\right)$ surjects onto the Fomin-Kirillov quadratic algebra $\mathcal{E}_{n}$ from [FK], which is relevant for embeddings of rational Cherednik algebras (see [BB, Example 7.24]). It is also quite surprising that when $\underline{\mathcal{H}}(W)$ is a negative braided Cherednik algebra from Theorem 0.2 with $W=B_{n}^{+}$, then the image $\varphi\left(S_{-\mathbf{1}}(V)\right)$ which is a subalgebra of $U^{-}$by Theorem 0.9, coincides with what Majid called the algebra of flat connections with constant coefficients in the noncommutative differential geometry of the symmetric group [M3].

To conclude the Introduction, we list relevant open problems and new directions of study.

Degenerate $q$-Hecke algebras. Here, the problem is two-fold:

Problem 1. (a) Given a q-symmetric algebra $S_{\mathbf{q}}(\tilde{V})$, find all finite groups $W \subset G L(V)$ such that the $W$-action on $V$ extends to the $W$-action on $S_{\mathbf{q}}(\tilde{V})$ by algebra automorphisms. 
(b) For each such $W$, find all flat deformations of the semidirect product algebra $S_{\mathbf{q}}(\tilde{V}) \rtimes \mathbb{C} W$.

Here, we solve Problem 1(a) in the case when all $q_{i j} \neq 1$ for $i \neq j$ and under the assumption that $W$ also acts on $S_{\mathbf{q}}(\tilde{V})^{*}$ by algebra automorphisms (Section $\left[2.3\right.$ ). In fact, the above groups $W_{\mathcal{C}, \mathcal{C}^{\prime}}$ form the most important class of solutions(when all $q_{i j}=-1$ for $j \neq i$ ).

Each q-Cherednik algebra and braided Cherednik algebra is a solution to Problem 1(b) in the case when $\tilde{V}=V \oplus V^{*}$. In our forthcoming paper [BB3] we construct more solutions to the problem.

Representations of braided Cherednik algebras. Similarly to the ordinary (rational) Cherednik algebras, one defines the category $\mathcal{O}$ for each braided Cherednik algebra $\underline{\mathcal{H}}$. The following natural problem emerges:

Problem 2. For each braided Cherednik algebra $\underline{\mathcal{H}}=\underline{\mathcal{H}}_{c}$, describe the category $\mathcal{O}$. In particular, find all values of parameters $c$ such that $\mathcal{O}$ contains finite-dimensional objects.

Even though $\mathcal{O}$ is not a tensor category, in addition to the Verma modules, it contains a number of interesting objects: $U^{-} \otimes \rho$, where $U^{-}$is the "generalised $r$-matrix algebra" from Theorem 0.9 and $\rho$ is any representation of $W$. As we mentioned above, if $W=S_{n}$ it is known from [BK] and BEER that the quadratic algebra $U^{-}$is Koszul. We expect this phenomenon to persist in general,

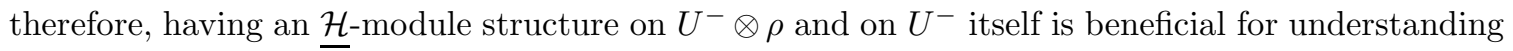
this quadratic algebra.

We plan to study finite-dimensional quotients of the Verma module $M_{\text {triv }}=S_{\mathbf{q}}(V)$ for $\underline{\mathcal{H}}$ in a separate paper. We expect that for negative braided Cherednik algebras the answer can be given along the lines of [BEG] and [VV]. And, according to Remark [0.4, the degenerate version of $\underline{\mathcal{H}}$ has a number of finite-dimensional modules that can be studied along the lines of $[\mathrm{G}$.

However, when $\underline{\mathcal{H}}$ is a braided tensor product (of negative braided or ordinary Cherednik algebras) as in (2), the representation category of $\underline{\mathcal{H}}$ is not at all determined by those of the tensor factors. For instance, by varying the matrix $\mathbf{q}$ and parameters $c_{i, \varepsilon}$ in (3) and Proposition 0.8 one can expect new interesting submodules of the Verma module $S_{\mathbf{q}}(V)$ even when $W=\mathbb{S}_{2} \times \mathbb{S}_{2} \times \cdots \times \mathbb{S}_{2}$. Another "degree of freedom" in representations of such factored $\underline{\mathcal{H}}$ is a choice of the field of definition $\mathbb{K} \subset \mathbb{C}$ containing all $q_{i j}$, say, $\mathbb{K}=\mathbb{Q}\left(q_{i j} \mid i, j=1, \ldots, n\right)$. Then, in the assumption that all $q_{i j}$ are roots of unity, i.e., $\mathbb{K}$ is a cyclotomic extension of $\mathbb{Q}$, there exist finite-dimensional quotients $\mathcal{B}_{\mathbf{q}}$ of $S_{\mathbf{q}}(V)$ and we expect that some of these $\mathcal{B}_{\mathbf{q}}$ are, in fact, representations of $\underline{\mathcal{H}}$. It follows from the famous Merkurjev-Suslin theorem that essentially all central simple algebras over $\mathbb{K}$ are simple finite-dimensional quotients of various $S_{\mathbf{q}}(V)$ so that an $\underline{\mathcal{H}}$-module structure on them would be of interest in $K$-theory.

Acknowledgments. We thank the organisers of the Workshop on Cherednik algebras at ICMS, Edinburgh, where this paper was started. The second author (A.B.) expresses his gratitude to the 
Mathematics Institute, University of Warwick for hospitality and support during his visit in Summer 2007. The authors are grateful to Weiqiang Wang for bringing to our attention the remarkable joint papers KW1, KW2 with Ta Khongsap.

\section{Quadratic Doubles}

In this section we introduce quadratic doubles as a sub-class of braided doubles over bialgebras (introduced and studied in our earlier paper [BB]) and present new results related specifically to quadratic doubles over group algebras. It is an open question, if (and how) results such as Proposition 1.15 and Theorem 1.21 can be extended to doubles over arbitrary bialgebras or Hopf algebras.

1.1. Triangular decomposition and braided doubles. Triangular decomposition of an associative algebra is defined as follows.

Definition 1.1. An algebra $A$ admits triangular decomposition $A=U^{-} \otimes U^{0} \otimes U^{+}$, if $U^{0}, U^{ \pm}$are subalgebras in $A$ such that $U^{-} U^{0}$ and $U^{0} U^{+}$are also subalgebras in $A$, and the vector space map

$$
U^{-} \otimes U^{0} \otimes U^{+} \rightarrow A, \quad u^{-} \otimes u^{0} \otimes u^{+} \mapsto u^{-} u^{0} u^{+} \in A,
$$

is bijective.

Let $V$ be a finite-dimensional module over a group $W$. To a linear map $\beta: V^{*} \otimes V \rightarrow \mathbb{C} W$ and two subspaces $R^{-} \subset T^{>0}(V), R^{+} \subset T^{>0}\left(V^{*}\right)$ we associate the algebra

$$
A_{\beta}\left(R^{-}, R^{+}\right)=\frac{T\left(V \oplus V^{*}\right) \rtimes \mathbb{C} W}{<R^{-}, R^{+},\left\{f \otimes v-v \otimes f-\beta(f \otimes v) \mid f \in V^{*}, v \in V\right\}>} .
$$

Here the symbol $\rtimes$ is used to denote a semidirect product. (If $A$ is a a $W$-module algebra, $A \rtimes$ $\mathbb{C} W$ is the algebra with underlying vector space $A \otimes \mathbb{C} W$ and multiplication $(a \otimes w)\left(a^{\prime} \otimes w^{\prime}\right)=$ $a w\left(a^{\prime}\right) \otimes w w^{\prime}$, where $a, a^{\prime} \in A$ and $w, w^{\prime} \in W$.) The angular brackets $<>$ denote the two-sided ideal with given generators.

Definition 1.2. The algebra $A_{\beta}\left(R^{-}, R^{+}\right)$is a braided double, if it has triangular decomposition

$$
A_{\beta}\left(R^{-}, R^{+}\right) \cong T(V) /<R^{-}>\otimes \mathbb{C W} \otimes T\left(V^{*}\right) /<R^{+}>.
$$

Remark 1.3. The algebra $A_{\beta}\left(R^{-}, R^{+}\right)$may either be a proper quotient of the vector space on the right, or be a braided double. In the latter case, $\left\langle R^{-}\right\rangle$is automatically a $W$-invariant ideal in the tensor algebra $T(V)$, and the subalgebra $T(V) /<R^{-}>\otimes \mathbb{C} W$ is isomorphic to $T(V) /<R^{-}>\rtimes \mathbb{C W}$; similarly for $R^{+}$. 
To understand braided doubles, one would like to study the locus of parameters $\left(\beta, R^{-}, R^{+}\right)$such that the algebra $A_{\beta}\left(R^{-}, R^{+}\right)$has triangular decomposition. The first major step is to determine for which $\beta$ braided doubles of the form $A_{\beta}\left(R^{-}, R^{+}\right)$exist. We say that $\beta: V^{*} \otimes V \rightarrow \mathbb{C} W$ is a $W$-equivariant map if $\beta$ is a $W$-homomorphism with respect to the standard diagonal $W$-action on $V^{*} \otimes V$ and the $W$-action on $\mathbb{C} W$ by conjugation.

Theorem 1.4. Let $V$ be a finite-dimensional module over a group $W$ and $\beta: V^{*} \otimes V \rightarrow \mathbb{C} W$ be a linear map. The algebra $A_{\beta}(0,0)$ is a braided double, if and only if $\beta$ is a $W$-equivariant map.

Proof. To prove the 'only if' part, pick any $f \in V^{*}, v \in V$ and $w \in W$. Using the relations $w v w^{-1}=w(v)$ and $w f w^{-1}=w(f)$ in the algebra $T\left(V \oplus V^{*}\right) \rtimes \mathbb{C} W$, write $\beta(w(f), w(v))=$ $\left(w f w^{-1}\right)\left(w v w^{-1}\right)-\left(w v w^{-1}\right)\left(w f w^{-1}\right)$, which is equal to $w(f v-v f) w^{-1}=w \beta(f, v) w^{-1}$. Thus, $\beta(w(f), w(v))=w \beta(f, v) w^{-1}$ holds in $A_{\beta}(0,0)$. Both sides of this relation lie in the group algebra $\mathbb{C} W$ which embeds injectively in $A_{\beta}(0,0)$ because of the triangular decomposition of $A_{\beta}(0,0)$. Hence the relation holds in $\mathbb{C} W$ and $\beta$ is $W$-equivariant. The (more difficult) 'if' part is proved in [BB Theorem 3.3]; the key point here is that the $W$-equivariance may be interpreted as the Yetter-Drinfeld condition for modules over a group algebra.

A braided double of the form $A_{\beta}(0,0)$ is called a free braided double and denoted $\widetilde{A}_{\beta}$. The proof of the Theorem implies that if $A_{\beta}\left(R^{-}, R^{+}\right)$is a braided double, then $\beta$ is $W$-equivariant and $A_{\beta}\left(R^{-}, R^{+}\right)$is a quotient of $\widetilde{A}_{\beta}$. The quotient map in question is a morphism in the category of braided doubles:

Definition 1.5 (The category $\left.\mathrm{D}_{W}\right)$. Denote by $\mathrm{D}_{\beta}(V)$ the set of braided doubles of the form $A_{\beta}\left(R^{-}, R^{+}\right)$. We introduce the category $\mathrm{D}_{W}$ such that

$$
\mathrm{Ob} \mathrm{D}_{W}=\bigcup_{V, \beta} \mathrm{D}_{\beta}(V)
$$

where the union is taken over all finite-dimensional $W$-modules $V$ and all $W$-equivariant maps $\beta: V^{*} \otimes V \rightarrow \mathbb{C} W$. If $A \in \mathrm{D}_{\beta}(U)$ and $B \in \mathrm{D}_{\gamma}(V)$, a morphism $\varphi: A \rightarrow B$ in $\mathrm{D}_{W}$ is an algebra map such that $\varphi(U) \subset V, \varphi\left(U^{*}\right) \subset V^{*}$ and $\left.\varphi\right|_{W}=\operatorname{id}_{W}$.

Clearly, $\varphi$ is uniquely determined by the two $W$-module maps $\mu=\left.\varphi\right|_{U}$ and $\nu=\left.\varphi\right|_{U^{*}}$. However, not every pair of $W$-module maps $U \stackrel{\mu}{\rightarrow} V, U^{*} \stackrel{\nu}{\rightarrow} V^{*}$ extends to an algebra homomorphism $A \rightarrow B$. For example, zero maps $\mu=\nu=0$ do not extend to a morphism between $A \in \mathrm{D}_{\beta}(U)$ and $B \in \mathrm{D}_{\gamma}(V)$, unless $\beta=0$.

Observe also that a braided double $A_{\beta}\left(R^{-}, R^{+}\right)$is a $W$-module algebra (where the action of $W$ on generators $w \in W$ of $A_{\beta}\left(R^{-}, R^{+}\right)$is by conjugation).

Using Lemma $4.4\left[\mathrm{BB}\right.$, one obtains a way to construct braided doubles in terms of $\widetilde{A}_{\beta}$ : 
Proposition 1.6. Let $R^{-} \subset T^{>0}(V)$ and $R^{+} \subset T^{>0}\left(V^{*}\right)$ be $W$-submodules such that $\left[R^{+}, V\right]=$ $\left[V^{*}, R^{-}\right]=0$ in the free braided double $\widetilde{A}_{\beta}$. Then $A_{\beta}\left(R^{-}, R^{+}\right)$is a braided double.

Remark 1.7. Based on this result, it is natural to expect that all braided doubles can be obtained as quotients of free braided doubles by the zero commutator condition in Proposition 1.6. For example, enveloping algebras $U(\mathfrak{g})$ and $U_{q}(\mathfrak{g})$ of a semisimple Lie algebra $\mathfrak{g}$ have such presentation (with $R^{ \pm}$being the Serre relations). Finding such "optimal" presentation for braided Heisenberg doubles [BB, 5.3] would imply interesting results on the structure of Nichols algebras. This optimal presentation is available for the main object of this section - quadratic doubles (see Theorem 1.8 below).

1.2. Quadratic doubles. A braided double $A_{\beta}\left(R^{-}, R^{+}\right)$in $\mathrm{D}_{\beta}(V)$ is called a quadratic double, if $R^{-} \subset V \otimes V$ and $R^{+} \subset V^{*} \otimes V^{*}$. Our original motivating example of this is rational Cherednik algebra; free braided doubles are quadratric too. We denote by $\mathrm{Q}_{\beta}(V)$ the set of quadratic doubles in $\mathrm{D}_{\beta}(V)$ and by $\mathrm{Q}_{W}$ the category of quadratic doubles over $\mathbb{C W}$ (a full subcategory of $\mathrm{D}_{W}$ ).

Theorem 1.8. Let $\beta: V^{*} \otimes V \rightarrow \mathbb{C} W$ be a $W$-equivariant map. Then $A_{\beta}\left(R^{-}, R^{+}\right)$is a quadratic double if and only if $R^{-} \subset V \otimes V, R^{+} \subset V^{*} \otimes V^{*}$ are $W$-submodules and

$$
\left[R^{+}, V\right]=0, \quad\left[V^{*}, R^{-}\right]=0 \quad \text { in the free double } \widetilde{A}_{\beta} .
$$

Proof. If $A_{\beta}\left(R^{-}, R^{+}\right)$is a quadratic double, the ideal $<R^{-}>$of $T(V)$ is $W$-invariant, hence so is its quadratic part $R^{-}$. The same applies to $R^{+}$. Furthermore, the relations in the free double $\widetilde{A}_{\beta}$ imply that the commutator $\left[V^{*}, R^{-}\right]$is a subspace of $V \otimes \mathbb{C W}$ which must obviously be in the kernel of the quotient map $\widetilde{A}_{\beta} \rightarrow A_{\beta}\left(R^{-}, R^{+}\right)$. The quotient map has no kernel in degrees less than 2 with respect to generators from $V$, thus $\left[V^{*}, R^{-}\right]=0$ in $\widetilde{A}_{\beta}$. The same argument applies to $\left[R^{+}, V\right]$ and thus establishes the 'only if' statement. The 'if' statement follows by Proposition 1.6

We denote

$$
R_{\beta \max }^{-}=\left\{r^{-} \in V \otimes V \mid\left[V^{*}, r^{-}\right]=0 \text { in } \widetilde{A}_{\beta}\right\}, \quad R_{\beta \max }^{+}=\left\{r^{+} \in V^{*} \otimes V^{*} \mid\left[r^{+}, V\right]=0 \text { in } \widetilde{A}_{\beta}\right\}
$$

It easy to see that as long as $\beta: V^{*} \otimes V \rightarrow \mathbb{C} W$ is $W$-equivariant (as above), $R_{\beta \text { max }}^{ \pm}$are $W$-invariant subspaces. This observation is useful in the following

Corollary 1.9. Let $\beta: V^{*} \otimes V \rightarrow \mathbb{C W}$ be a $W$-equivariant map. The algebras $\widetilde{A}_{\beta}:=A_{\beta}(0,0)$ and $A_{\beta}:=A_{\beta}\left(R_{\beta \max }^{-}, R_{\beta \max }^{+}\right)$are quadratic doubles in $\mathrm{Q}_{\beta}(V)$. For any quadratic double $A \in \mathrm{Q}_{\beta}(V)$ there are quotient maps $\widetilde{A}_{\beta} \rightarrow A \rightarrow A_{\beta}$ in $\mathrm{Q}_{W}$. 
Definition 1.10. The quadratic double $A_{\beta} \cong T(V) /<R_{\beta \text { max }}^{-}>\otimes \mathbb{C} W \otimes T\left(V^{*}\right) /<R_{\beta \text { max }}^{+}>$in $\mathrm{Q}_{\beta}(V)$ is called the minimal quadratic double with parameter $\beta \in\left\{W\right.$-equivariant maps $V^{*} \otimes V \rightarrow$ $\mathbb{C} W\}$.

Of the objects $\mathrm{Q}_{\beta}(V)$, it is the minimal quadratic double $A_{\beta}$ that is most interesting algebraically. The quadratic relations in $A_{\beta}$ are given implicitly as kernels of certain linear operators (see Lemma 1.16 below). The central problem in the theory of quadratic doubles is two-fold:

Problem 1.11. Let $V$ be a finite-dimensional module for a group $W$. Given a $W$-equivariant map $\beta: V^{*} \otimes V \rightarrow \mathbb{C} W$, define the algebra $A_{\beta}$ explicitly by generators and relations (i.e., find an explicit description of $\left.R_{\beta \text { max }}^{ \pm}\right)$.

Problem 1.12. Given $W$-submodules $R^{-} \subset V \otimes V$ and $R^{+} \subset V^{*} \otimes V^{*}$, find all maps $\beta: V^{*} \otimes V \rightarrow$ $\mathbb{C} W$ such that $A_{\beta}\left(R^{-}, R^{+}\right)$is a quadratic double.

Problem 1.12 is in fact a deformation question. Regard $A_{\beta}\left(R^{-}, R^{+}\right)$as a deformation, with parameter $\beta$, of the algebra $A_{0}\left(R^{-}, R^{+}\right)$; the latter is a quadratic double by Theorem 1.8 . One needs to find the values of $\beta$ for which the deformation is flat (the flatness locus).

Example 1.13. When $W \subset \mathrm{GL}(V), R^{-}=\wedge^{2} V \subset V \otimes V$ and $R^{+}=\wedge^{2} V^{*} \subset V^{*} \otimes V^{*}$, the solution to Problem 1.12 is given by rational Cherednik algebras $A_{\beta}\left(R^{-}, R^{+}\right)$with

$$
\beta(\xi \otimes v)=\langle v, \xi\rangle+\sum_{s} c_{s}\left\langle v, \alpha_{s}^{\vee}\right\rangle\left\langle\alpha_{s}, \xi\right\rangle s
$$

for $\xi \in V^{*}, v \in V$; cf. [EG]. The sum is taken over all complex reflections $s \in W$, the parameters $c=\left\{c_{s}\right\}, c_{s} \in \mathbb{C}$ satisfy $c_{w s w^{-1}}=c_{s}$ for all $w \in W$, and $\alpha_{s} \in V, \alpha_{s}^{\vee} \in V^{*}$ is the root-coroot pair for the complex reflection $s$, meaning that $s(v)=v-\left\langle v, \alpha_{s}^{\vee}\right\rangle \alpha_{s}$ for all $v \in V$. Here $\langle v, \xi\rangle$ can be any $W$-invariant pairing between $V$ and $V^{*}$. If it is the standard evaluation pairing, denote the corresponding rational Cherednik algebra by $H_{c}(W)$, whereas if $\langle v, \xi\rangle=0$, denote the corresponding algebra by $H_{0, c}(W)$.

1.3. Operations $\diamond$ and $\star$ on quadratic doubles. Recall that the parameter $\beta$ in a quadratic double $A_{\beta}\left(R^{-}, R^{+}\right)$belongs to the space of $W$-equivariant linear maps from $V^{*} \otimes V$ to $\mathbb{C} W$. Let us now observe that this parameter space has the structure of an algebra. Write $\beta$ in the form

$$
\beta(f \otimes v)=\sum_{w \in W}\left\langle L_{w}(v), f\right\rangle w, \quad f \in V^{*}, v \in V,
$$

where $L_{w} \in \operatorname{End}(V)$ are zero for all but finitely many $w \in W$. We identify $\beta$ with the element $\sum_{w \in W} \delta_{w} \otimes L_{w}$ of the algebra

$$
\left(\mathbb{C}(W)_{0} \otimes \operatorname{End}(V)\right)^{W}
$$


Here $\mathbb{C}(W)_{0}$ is the algebra, with respect to pointwise multiplication, of complex-valued functions on $W$ with finite support. It is spanned by delta-functions $\delta_{w}, w \in W$. The action of $W$ on $\mathbb{C}(W)_{0}$ is by conjugation: $w\left(\delta_{\sigma}\right)=\delta_{w \sigma w^{-1}}$. The $\otimes$ is the standard tensor product of algebras, where the tensorands commute. The algebra $\left(\mathbb{C}(W)_{0} \otimes \operatorname{End}(V)\right)^{W}$ of parameters contains an identity if and only if the group $W$ is finite.

Let $\beta=\sum_{w} \delta_{w} \otimes L_{w}, \gamma=\sum_{w} \delta_{w} \otimes M_{w}$ be elements of $\left(\mathbb{C}(W)_{0} \otimes \operatorname{End}(V)\right)^{W}$. We observe that their sum and product in the algebra of parameters are rewritten as linear maps from $V^{*} \otimes V$ to $\mathbb{C} W$ as follows:

$$
(\beta+\gamma)(f \otimes v)=\sum_{w \in W}\left\langle\left(L_{w}+M_{w}\right)(v), f\right\rangle w, \quad(\beta \gamma)(f \otimes v)=\sum_{w \in W}\left\langle\left(L_{w} M_{w}\right)(v), f\right\rangle w .
$$

Let

$$
\mathrm{Q}(V):=\bigsqcup_{\beta \in\left(\mathbb{C}(W)_{0} \otimes \operatorname{End}(V)\right)^{W}} \mathrm{Q}_{\beta}(V)
$$

be the set of all quadratic doubles of the $W$-module $V$. We will now see how the above sum and product can be "lifted" from the algebra of parameters to $\mathrm{Q}(V)$, to yield two operations, $\diamond$ and $\star$.

Definition 1.14. Let $A=A_{\beta}\left(R^{-}, R^{+}\right)$and $B=A_{\gamma}\left(S^{-}, S^{+}\right)$be quadratic doubles in $\mathrm{Q}(V)$. Denote

$$
A \diamond B=A_{\beta+\gamma}\left(R^{-} \cap S^{-}, R^{+} \cap S^{+}\right), \quad A \star B=A_{\beta \gamma}\left(S^{-}, R^{+}\right) .
$$

Proposition 1.15. If $A$ and $B$ are quadratic doubles in $\mathrm{Q}(V)$, then $A \diamond B$ and $A \star B$ are also quadratic doubles in $\mathrm{Q}(V)$.

The proposition will follow from a technical

Lemma 1.16. Let $\beta=\sum_{w} \delta_{w} \otimes L_{w}$ be an element of the parameter algebra $\left(\mathbb{C}_{0}(W) \otimes \operatorname{End}(V)\right)^{W}$. The quadratic relations in the minimal quadratic double $A_{\beta}$ are given by

$$
R_{\beta \text { max }}^{-}=\cap_{w \in W} \operatorname{ker} T_{w, \beta}^{-}, \quad R_{\beta \text { max }}^{+}=\cap_{w \in W} \operatorname{ker} T_{w, \beta}^{+},
$$

where $T_{w, \beta}^{-} \in \operatorname{End}(V \otimes V)$ and $T_{w, \beta}^{+} \in \operatorname{End}\left(V^{*} \otimes V^{*}\right)$ are defined by

$$
T_{w, \beta}^{-}(u \otimes v)=\left(L_{w} \otimes \operatorname{id}_{V}\right)(u \otimes w(v)+v \otimes u), \quad T_{w, \beta}^{+}(f \otimes g)=\left(\operatorname{id}_{V^{*}} \otimes L_{w}^{*}\right)\left(w^{-1}(f) \otimes g+g \otimes f\right) .
$$

Proof. Recall that $R_{\beta \max }^{-}$is defined, following Theorem 1.8, as the space of quadratic tensors in $V \otimes V$ that commute, in the free double $\widetilde{A}_{\beta}$, with all elements of $V^{*}$. By the Leibniz rule, the commutator of $f \in V^{*}$ with $u \otimes v \in V \otimes V$ in $\widetilde{A}_{\beta}$ is

$$
\begin{aligned}
\sum_{w \in W}\left\langle L_{w}(u), f\right\rangle w v+u\left\langle L_{w}(v), f\right\rangle w & =\sum_{w \in W}\left(\left\langle L_{w}(u), f\right\rangle w(v)+\left\langle L_{w}(v), f\right\rangle u\right) \otimes w \\
& =\sum_{w \in W}\left(\langle\cdot, f\rangle \otimes \operatorname{id}_{V}\right) T_{w, \beta}^{-}(u \otimes v) \otimes w
\end{aligned}
$$


hence indeed $R_{\beta \text { max }}^{-}=\cap_{w \in W} \operatorname{ker} T_{w, \beta}^{-}$. The argument for $R_{\beta \text { max }}^{-}$is similar.

Proof of Proposition 1.15, To establish that $A \diamond B$ is a quadratic double, we need to show that $R^{ \pm} \cap S^{ \pm}$is a $W$-submodule of $R_{\beta+\gamma \max }^{ \pm}$and to apply Theorem 1.8. But clearly $T_{w, \beta+\gamma}^{ \pm}=T_{w, \beta}^{ \pm}+T_{w, \gamma}^{ \pm}$, thus $\operatorname{ker} T_{w, \beta+\gamma}^{ \pm}$contains the intersection of $\operatorname{ker} T_{w, \beta}^{ \pm}$and $\operatorname{ker} T_{w, \gamma}^{ \pm}$, which contains $R^{ \pm} \cap S^{ \pm}$. The latter is a $W$-submodule as an intersection of $W$-submodules. In a similar fashion, to show that $A \star B$ is a quadratic double, we need to check that $S^{-} \subset \operatorname{ker} T_{w, \beta \gamma}^{-}$and $R^{+} \subset \operatorname{ker} T_{w, \beta \gamma}^{+}$for all $w \in W$. Write $\beta=\sum_{w \in W} \delta_{w} \otimes L_{w}$ and $\gamma=\sum_{w \in W} \delta_{w} \otimes M_{w}$. Observe that $T_{w, \beta \gamma}^{-}=\left(L_{w} \otimes \mathrm{id}_{V}\right) T_{w, \gamma}^{-}$, therefore the kernel of $T_{w, \beta \gamma}^{-}$contains that of $T_{w, \gamma}^{-}$, which contains $S^{-}$. Furthermore, $T_{w, \beta \gamma}^{+}=\left(\operatorname{id}_{V^{*}} \otimes M_{w}^{*}\right) T_{w, \beta}^{+}$, hence its kernel contains that of $T_{w, \beta}^{+}$, which contains $R^{+}$.

Remark 1.17. The two operations $\diamond, \star: \mathrm{Q}(V) \times \mathrm{Q}(V) \rightarrow \mathrm{Q}(V)$ satisfy the following axioms:

$$
\begin{aligned}
& (A \diamond B) \diamond C=A \diamond(B \diamond C), \quad(A \star B) \star C=A \star(B \star C) ; \\
& A \diamond B=B \diamond A ; \\
& A_{0} \diamond A=A \diamond A_{0}=A ; \\
& A \star(B \diamond C)=(A \star B) \diamond(A \star C), \quad(A \diamond B) \star C=(A \star C) \diamond(B \star C),
\end{aligned}
$$

where $A_{0} \cong V \otimes \mathbb{C W} \otimes V^{*}$ is the minimal quadratic double corresponding to $\beta=0$ (the "smallest possible" quadratic double). This is a subset of the semiring axioms, however, note that there is no zero or identity element with respect to $\star$.

Warning: the operations $\diamond$ and $\star$ do not preserve the minimality of quadratic doubles: $A \diamond B$ and $A \star B$ may not be minimal even if $A, B$ are both minimal.

We will now see how the operation $\diamond$ "behaves" with respect to morphisms in $\mathrm{Q}_{W}$.

Proposition 1.18. Let $U, V$ be two finite-dimensional $W$-modules, and assume that $A, B \in \mathrm{Q}(U)$, $A^{\prime}, B^{\prime} \in \mathrm{Q}(V)$ are quadratic doubles. If a pair $U \stackrel{\mu}{\rightarrow} V, U^{*} \stackrel{\nu}{\rightarrow} V^{*}$ of $W$-module maps extends to a morphism $\varphi: A \rightarrow A^{\prime}$ and to a morphism $\psi: B \rightarrow B^{\prime}$ in $\mathrm{Q}_{W}$, then the same maps $\mu, \nu$ extend to a morphism

$$
\varphi \diamond \psi: A \diamond B \rightarrow A^{\prime} \diamond B^{\prime}
$$

Proof. Let $A=A_{\beta}\left(R^{-}, R^{+}\right), B=A_{\gamma}\left(S^{-}, S^{+}\right)$where $\beta, \gamma: U^{*} \otimes U \rightarrow \mathbb{C} W$ are $W$-equivariant maps, $R^{-}, S^{-} \subset U \otimes U$ and $R^{+}, S^{+} \subset U^{*} \otimes U^{*}$. Let $A^{\prime}=A_{\beta^{\prime}}\left(R^{\prime^{-}}, R^{\prime+}\right), B^{\prime}=A_{\gamma^{\prime}}\left({S^{\prime}}^{-}, S^{\prime+}\right)$, similarly to $A, B$ but with $U$ replaced with $V$. Looking at the relations in quadratic doubles, we conclude that $\mu, \nu$ extend to algebra homomorphisms $A \rightarrow A^{\prime}, B \rightarrow B^{\prime}$ if and only if $\beta=\beta^{\prime} \circ(\nu \otimes \mu)$, $\gamma=\gamma^{\prime} \circ(\nu \otimes \mu)$ and

$$
(\mu \otimes \mu) R^{-} \subset R^{\prime^{-}}, \quad(\mu \otimes \mu) S^{-} \subset{S^{\prime}}^{-}, \quad(\nu \otimes \nu) R^{+} \subset R^{\prime+}, \quad(\nu \otimes \nu) S^{+} \subset{S^{\prime}}^{+} .
$$


But then $\beta+\gamma=\left(\beta^{\prime}+\gamma^{\prime}\right) \circ(\nu \otimes \mu)$ and $(\mu \otimes \mu)\left(R^{-} \cap S^{-}\right) \subset\left(R^{--} \cap S^{\prime^{-}}\right)$, similarly for $R^{+} \cap S^{+}$. Thus, $\mu, \nu$ extend to a morphism $A \diamond B \rightarrow A^{\prime} \diamond B^{\prime}$, which we denote by $\varphi \diamond \psi$.

1.4. Yetter-Drinfeld modules. Yetter-Drinfeld modules over $W$ provide a family of deformation parameters $\beta$, for which the minimal quadratic doubles $A_{\beta}$ have a nice description and are in a sense universal, as many quadratic doubles can be realised as their subalgebras (see Theorem 1.21 below). Let us recall the definition of a Yetter-Drinfeld module. When the group $W$ is finite, it is the same as a module over the Hopf algebra $D(W)$, the Drinfeld quantum double of $W$.

Definition 1.19. A Yetter-Drinfeld module for a group $W$ is a $W$-module $Y$ with a grading $Y=$ $\bigoplus_{w \in W} Y_{w}$, such that $\sigma\left(Y_{w}\right)=Y_{\sigma w \sigma^{-1}}$ for all $w, \sigma \in W$.

Whenever $Y$ is a Yetter-Drinfeld (YD-) module over $W$, we denote by $|y|$ the $W$-degree of homogeneous $y \in Y$. When the notation $|\cdot|$ is used in formulas, extension from homogeneous elements to all elements of $Y$ by linearity is implied. For example, the Yetter-Drinfeld axiom may be written as $|w(y)|=w|y| w^{-1}$.

Clearly, if $Y$ is finite-dimensional, the dual module $Y^{*}$ is a YD-module via $Y^{*}=\bigoplus_{w \in W}\left(Y^{*}\right)_{w}$ with $\left(Y^{*}\right)_{w}=\operatorname{Hom}_{\mathbb{C}}\left(Y_{w^{-1}}, \mathbb{C}\right)$. Define the linear map $\beta_{Y}: Y^{*} \otimes Y \rightarrow \mathbb{C} W$ by

$$
\beta_{Y}(f \otimes v)=\langle v, f\rangle|v|
$$

It is straightforward to verify that the map $\beta_{Y}$ is $W$-equivariant.

1.5. Heisenberg quadraic doubles. To each finite-dimensional Yetter-Drinfeld module $Y$ over $W$ is therefore associated a minimal quadratic double $A_{Y}:=A_{\beta_{Y}}$, referred to as the Heisenberg quadratic double of $Y$.

To describe Heisenberg quadratic doubles more explicitly, recall that the linear map

$$
\Psi_{Y}: Y \otimes Y \rightarrow Y \otimes Y, \quad \Psi_{Y}(y \otimes z)=|y|(z) \otimes y
$$

is a braiding on $Y$, i.e., a solution to the braid equation; see [BB Section 5]. Viewing $Y^{*} \otimes Y^{*}$ as a dual space to $Y \otimes Y$, denote by $\Psi_{Y}^{*}$ the adjoint map to $\Psi_{Y}$. (This braiding on $Y^{*}$ is not the same as the braiding $\Psi_{Y^{*}}$ given by the YD-module structure on $Y^{*}$; the two are related via $\Psi_{Y}^{*}=\tau \circ \Psi_{Y^{*}} \circ \tau$, where $\tau(x \otimes y)=y \otimes x$ is the trivial braiding.) Furthermore, any braiding $\Psi \in \operatorname{End}(V \otimes V)$ on a vector space $V$ gives rise to a braided analogue of the symmetric algebra of $V$ :

$$
S(V, \Psi)=T(V) /<\operatorname{ker}\left(\operatorname{id}_{V \otimes V}+\Psi\right)>
$$

of which $S(V)$ is a particular case corresponding to $\Psi=\tau$. Theorem 5.4 in $[\mathrm{BB}$ implies 
Proposition 1.20. The Heisenberg quadratic double $A_{Y}$ has triangular decomposition

$$
A_{Y}=S\left(Y, \Psi_{Y}\right) \otimes \mathbb{C} W \otimes S\left(Y^{*}, \Psi_{Y}^{*}\right) .
$$

The crucial property of Heisenberg quadratic doubles is given in

Theorem 1.21. For any finite-dimensional $W$-module $V$ and any two quadratic doubles $A, B$ in $\mathrm{Q}(V)$, there exists a finite-dimensional Yetter-Drinfeld module $Y$ over $W$ and a morphism $A \star B \rightarrow$ $A_{Y}$ in $\mathrm{Q}_{W}$.

Proof. Let $A=A_{\beta}\left(R^{-}, R^{+}\right)$and $B=A_{\gamma}\left(S^{-}, S^{+}\right)$, where $\beta, \gamma$ are $W$-equivariant maps from $V^{*} \otimes V$ to $\mathbb{C} W$ given by $\beta(f \otimes v)=\sum_{w \in W}\left\langle L_{w}(v), f\right\rangle w, \gamma(f \otimes v)=\sum_{w \in W}\left\langle M_{w}(v), f\right\rangle w$ with $L_{w}, M_{w} \in$ $\operatorname{End}(V)$. The finite subset $E=\left\{g \in W \mid L_{g} \neq 0\right.$ or $\left.M_{g} \neq 0\right\}$ of $W$ is conjugation-invariant by the $W$-equivariance of $\beta, \gamma$. Denote by $\mathbb{C} E$ the linear span of $E$ in $\mathbb{C} W$. We introduce the space $Y$ equipped with $W$-action and $W$-grading by

$$
Y=\mathbb{C} E \otimes V, \quad w(g \otimes v)=w g w^{-1} \otimes w(v), \quad|g \otimes v|=g
$$

for all $g \in E, v \in V, w \in W$. It is easy to see that $Y$ is a Yetter-Drinfeld module for $W$. The dual Yetter-Drinfeld module $Y^{*}$ can also be described explicitly: $Y^{*}=\mathbb{C} E^{-1} \otimes V^{*}, w(h \otimes f)=$ $w h w^{-1} \otimes w(f),|h \otimes f|=h$ for all $h \in E^{-1}=\left\{g^{-1} \mid g \in E\right\}, f \in V^{*}$ and $w \in W$. One checks that $\langle g \otimes v, h \otimes f\rangle=\delta_{g^{-1}, h}\langle v, f\rangle$ is a pairing between $Y$ and $Y^{*}$ that indeed makes $Y^{*}$ the YD module dual to $Y$. The maps $\mu: V \rightarrow Y, \nu: V^{*} \rightarrow Y^{*}$ given by

$$
\mu(v)=\sum_{w \in W} w \otimes M_{w}(v), \quad \nu(f)=\sum_{w \in W} w^{-1} \otimes L_{w}^{*}(f)
$$

are $W$-module homomorphisms because $\gamma, \beta$ are $W$-equivariant.

It remains to show that $\mu, \nu$ extend to a morphism between the quadratic doubles $A \star B=$ $A_{\beta \gamma}\left(S^{-}, R^{+}\right)$and $A_{Y}$. As in the proof of Proposition 1.18, it is enough to show that

$$
\beta \gamma=\beta_{Y} \circ(\nu \otimes \mu), \quad(\mu \otimes \mu) S^{-} \subset \operatorname{ker}\left(\operatorname{id}_{Y} \otimes Y+\Psi_{Y}\right), \quad(\nu \otimes \nu) R^{+} \subset \operatorname{ker}\left(\operatorname{id}_{Y^{*} \otimes Y^{*}}+\Psi_{Y}^{*}\right) .
$$

Since $\beta_{Y}(h \otimes f \otimes g \otimes v)=\delta_{g, h^{-1}}\langle v, f\rangle g$ where $g, h^{-1} \in E, f \in V^{*}, v \in V$, one has $\beta_{Y}(\nu(f) \otimes \mu(v))=$ $\sum_{w \in E}\left\langle M_{w}(v), L_{w}^{*}(f)\right\rangle w=\sum_{w}\left\langle\left(L_{w} M_{w}\right)(v), f\right\rangle w=(\beta \gamma)(f \otimes v)$ as required. The remaining two equalities are established by applying Lemma 1.16 (similarly to the proof of [BB, Theorem 6.9]). The Theorem is proved.

1.6. Generalised Dunkl operators. We now look at the Dunkl operators in the context of quadratic doubles and propose their generalisation. 
Let $V$ be a finite-dimensional module over a group $W$ and $A=U^{-} \otimes \mathbb{C} W \otimes U^{+}$be a quadratic double in $\mathrm{Q}(V)$. The subalgebra $\mathbb{C} W \otimes U^{+}$of $A$ has one-dimensional trivial representation triv, where $w \in W$ acts by 1 and $V$ acts by 0 . One has the induced representation of $A$ :

$$
\operatorname{Ind}_{\mathbb{C} W \otimes U^{+}}^{A}(\text { triv }) \cong U^{-} \text {as vector spaces. }
$$

Denote the action of $\xi \in V^{*}$ on $U^{-}$via this representation by $\partial_{\xi}$. The operators $\partial_{\xi}: U^{-} \rightarrow U^{-}$are of degree -1 with respect to the grading in $U^{-}$.

In Heisenberg quadratic doubles, the operators $\partial_{\xi}$ are called braided derivatives. They can be computed as follows. Suppose that $Y=\oplus_{w \in W} Y_{w}$ is a Yetter-Drinfeld module for $W$. Let $\left\{y_{w}^{i} \in Y_{w} \mid\right.$ $\left.w \in W, i=1, \ldots, \operatorname{dim} Y_{w}\right\}$ be a basis of $Y$ compatible with the $W$-grading, and let $f_{w}^{i} \in\left(Y^{*}\right)_{w^{-1}}$ form the dual basis of $Y^{*}$. One shows that the braided derivatives

$$
\partial_{w}^{i}=\partial_{f_{w}^{i}}: S\left(Y, \Psi_{Y}\right) \rightarrow S\left(Y, \Psi_{Y}\right)
$$

satisfy, and are determined by, the following properties:

(i) $\partial_{w}^{i} y_{\sigma}^{j}=\delta_{i, j} \delta_{w, \sigma}$

(ii) ( $w$-twisted Leibniz rule) $\partial_{w}^{i}(a b)=\left(\partial_{w}^{i} a\right) w(b)+a\left(\partial_{w}^{i} b\right)$ for all $a, b \in S\left(Y, \Psi_{Y}\right)$.

Let us now consider a quadratic double $A=U^{-} \otimes \mathbb{C} W \otimes U^{+}$in $\mathrm{Q}(V)$ which is not Heisenberg. For emphasis, we will now write $\nabla_{\xi}$ instead of $\partial_{\xi}$ in $A$ and call $\nabla_{\xi}$ generalised Dunkl operators.

Suppose that $A$ embeds in a Heisenberg quadratic double $A_{Y}$ for some Yetter-Drinfeld module $Y$, and let $V \stackrel{\mu}{\rightarrow} Y, V^{*} \stackrel{\nu}{\rightarrow} Y^{*}$ be a pair of maps which give rise to such embedding. Put

$$
\alpha_{w}^{i}:=\nu^{*}\left(y_{w}^{i}\right) \in V, \quad \alpha_{w}^{\vee i}:=\mu^{*}\left(f_{w}^{i}\right) \in V^{*}, \quad i=1, \ldots, \operatorname{dim} Y_{w} .
$$

The vectors $\alpha_{w}^{i}$, respectively $\alpha^{\vee}{ }_{w}^{i}$, are an analogue of roots, respectively coroots, of the group $W$. Initial data for this generalised root system is a $W$-module $V$ equipped with $W$-homomorphism $\beta$ from $V^{*} \otimes V$ to the adjoint representation of $W$. Strictly speaking, the construction depends on a particular "quantisation" $Y$ of $(V, \beta)$, but one hopes that the "root system" has a reasonable uniqueness property; we do not consider this question here. It is not difficult to see that the generalised Dunkl operators are expressed as

$$
\nabla_{\xi}=\sum_{w \in W} \sum_{i=1}^{\operatorname{dim} Y_{w}}\left\langle\alpha_{w}^{i}, \xi\right\rangle \bar{\partial}_{w}^{i},
$$

with $\bar{\partial}_{w}^{i}: U^{-} \rightarrow U^{-}$defined by its two properties,

$$
\bar{\partial}_{w}^{i}(v)=\left\langle v, \alpha^{\vee}{ }_{w}^{i}\right\rangle, \quad v \in V ; \quad \bar{\partial}_{w}^{i} \quad \text { satisfies the } w \text {-twisted Leibniz rule. }
$$


1.7. Braided reduction of quadratic doubles. To conclude this Section, we describe a method of obtaining a wider class of algebras with triangular decomposition over $\mathbb{C} W$ as subalgebras in quadratic doubles. Recall that if $Y$ is a finite-dimensional Yetter-Drinfeld module over $W$, then $\Psi_{Y}$ is the braiding $y \otimes z \mapsto|y|(z) \otimes y$ on $Y$, and $\tau$ is the trivial braiding. The spaces $Y^{*}, Y^{*} \otimes Y^{*}$ etc. are also YD-modules, and the $W$-grading on $Y^{*} \otimes Y^{*}$ is by $|f \otimes g|=|f||g|$.

Proposition 1.22. Assume that a $W$-module $V$ has $W$-grading which makes $V$ a Yetter-Drinfeld module. Let $A=A_{\beta}\left(R^{-}, R^{+}\right)$be a quadratic double in $\mathrm{Q}_{\beta}(V)$, such that $\tau\left(R^{+}\right)$is a $W$-graded subspace of $V^{*} \otimes V^{*}$. Let

$$
\underline{A}=\frac{T\left(V \oplus V^{*}\right) \rtimes \mathbb{C} W}{<R^{-}, \underline{R}^{+},\left\{\underline{[\theta, v]}-|\theta| \beta(\theta \otimes v): \theta \in V^{*}, v \in V\right\}>},
$$

where $\underline{[f, v]}=f \otimes v-|f|(v) \otimes f$ is the braided commutator between $V^{*}$ and $V$, and $\underline{R}^{+}=\left(\Psi_{\left.V^{*} \circ \tau\right) R^{+}}\right.$. Then there exists an algebra isomorphism $t: \underline{A} \rightarrow A$, given on generators of $\underline{A}$ by

$$
\left.t\right|_{V}=\operatorname{id}_{V},\left.\quad t\right|_{W}=\operatorname{id}_{W}, \quad t(\theta)=|\theta| \cdot \theta, \quad \theta \in V^{*} .
$$

Proof. First, we have to show that $t$ maps relations in $A$ to relations in $A$. For $\theta \in V^{*}$ we have $t\left(w \theta w^{-1}-w(\theta)\right)=w \cdot|\theta| \theta \cdot w^{-1}-|w(\theta)| w(\theta)$. This is a relation in $A$, as $|w(\theta)|=w|\theta| w^{-1}$ by the Yetter-Drinfeld condition on $V^{*}$. Furthermore, $t(\underline{[\theta, v]})=|\theta| \theta v-|\theta|(v) \cdot|\theta| \theta=|\theta|(\theta v-v \theta)$ which in $A$ coincides with $|\theta| \beta(\theta, v)$.

Now observe that $\Psi_{V^{*}}(\tau(\theta \otimes \kappa))=|\kappa|(\theta) \otimes \kappa$ for $\theta \otimes \kappa \in V^{*} \otimes V^{*}$. This is mapped by $t$ to $|\kappa||\theta||\kappa|^{-1} \cdot|\kappa|(\theta) \cdot|\kappa| \kappa=|\kappa||\theta| \cdot \theta \kappa$. Hence if $a \in R^{+}$is such that $\tau(a)$ is $W$-homogeneous (such $a$ span $\left.R^{+}\right)$, then $t\left(\Psi_{V^{*}} \tau(a)\right)=|\tau(a)| a$. Thus, $t\left(R^{+}\right) \subset \mathbb{C} W \otimes R^{+}$. It remains to note that the relations $w v w^{-1}-w(v)$ and $R^{-}$in $\underline{A}$ are mapped by $t$ to exactly the same relations in $A$. We conclude that $t$ is a map of algebras.

In the same fashion one shows that the map $t^{-1}$, given on generators of $A$ by $\left.t^{-1}\right|_{V}=\mathrm{id} V$, $\left.t^{-1}\right|_{W}=\operatorname{id}_{W}, t^{-1}(f)=|f|^{-1} \cdot f, f \in V^{*}$, is an algebra homomorphism from $A$ to $\underline{A}$. As $t t^{-1}$ and $t^{-1} t$ are identity on generators, $t^{-1}$ is the inverse of $t$.

Remark 1.23. It is easy to deduce from the Proposition that the algebra $\underline{A}$, given by its presentation, has triangular decomposition $\underline{A}=T(V) /<R^{-}>\otimes \mathbb{C} W \otimes T\left(V^{*}\right) /<\underline{R}^{+}>$.

Definition 1.24. In the above notation, assume that for all $\theta \in V^{*}, v \in V$ the braided commutator $\underline{[\theta, v]}$ in $\underline{A}$ lies in $\mathbb{C} W^{\prime}$ for some subgroup $W^{\prime}$ of $W$. The algebra $A=\underline{A}$ has subalgebra $\underline{A}^{\prime} \cong$ $\overline{T(V)} /<R^{-}>\otimes \mathbb{C} W^{\prime} \otimes T\left(V^{*}\right) /<\underline{R}^{+}>$. We call $\underline{A}^{\prime}$ a braided reduction of $A$.

Example 1.25 (Braided Weyl algebra). To show how the braided reduction works, we consider the "extreme" example which is $A=A_{V}$, the Heisenberg quadratic double of a Yetter-Drinfeld module 
$V$ over $W$. We compute the braided commutator in $\underline{A}$ of $\theta \in V^{*}$ and $v \in V$ :

$$
\underline{[\theta, v]}=|\theta| \beta_{V}(\theta, v)=|\theta| \cdot\langle v, \theta\rangle \cdot|v|=\langle v, \theta\rangle \cdot 1,
$$

as $\langle v, \theta\rangle \neq 0$ for $W$-homogeneous $\theta$, $v$ only if $|\theta|=|v|^{-1}$. We thus have a braided reduction $\underline{A}^{\prime} \cong T(V) /<R^{-}>\otimes \mathbb{C} \cdot 1 \otimes T\left(V^{*}\right) /<\underline{R}^{+}>$of $A$. Furthermore, using $\Psi_{V^{*}}=\tau \circ \Psi_{V}^{*} \circ \tau$ we find $\underline{R}^{+}=\left(\Psi_{V^{*}} \tau\right) \operatorname{ker}\left(\mathrm{id}+\tau \Psi_{V^{*}} \tau\right)=\operatorname{ker}\left(\mathrm{id}+\Psi_{V^{*}}\right)$. Hence

$$
\underline{A}_{V}:=\underline{A}^{\prime} \cong S\left(V, \Psi_{V}\right) \otimes S\left(V^{*}, \Psi_{V^{*}}\right)
$$

with defining commutation relation $\theta v-|\theta|(v) \theta=\langle v, \theta\rangle \cdot 1$ between $\theta \in V^{*}$ and $v \in V$. We have $A_{V} \cong \underline{A}_{V} \rtimes \mathbb{C W}$ as algebras. The algebra $\underline{A}_{V}$ is a particular case of Majid's braided Weyl algebra M1, hence

Definition 1.26. $\underline{A}_{V}$ is called the braided Weyl algebra of the Yetter-Drinfeld module $V$.

\section{2. q-CHEREDNIK ALGEBRAS}

In this Section we introduce q-Cherednik algebras. They are quadratic doubles, which allows us to use the methods of Section 1 . On the other hand, results about q-Cherednik algebras will be translated to braided Cherednik algebras, obtained from q-Cherednik algebras by braided reduction.

2.1. The q-polynomial algebra. Recall that a rational Cherednik algebra of a finite linear group $W \subset \mathrm{GL}(V)$ is a deformation of the semidirect product $\mathcal{D}(V) \rtimes \mathbb{C} W$, where $\mathcal{D}(V) \cong S(V) \otimes S\left(V^{*}\right)$ is the Weyl algebra of the space $V$. Our aim is to replace the polynomial algebra $S(V)$ with its $q$-analogue. Throughout, $V=\operatorname{span}\left(x_{1}, \ldots, x_{n}\right)$ will be a space spanned by $n$ independent variables, and the symbol $\mathbf{q}$ will stand for an $n \times n$ matrix of deformation parameters $q_{i j}$ such that $q_{i i}=1$, $q_{i j} q_{j i}=1$ for all $i, j=1, \ldots, n$. The q-polynomial algebra

$$
S_{\mathbf{q}}(V):=\mathbb{C}\left\langle x_{1}, \ldots, x_{n} \mid x_{i} x_{j}=q_{i j} x_{j} x_{i}\right\rangle
$$

is a flat deformation of the symmetric algebra $S(V)$. The space of quadratic relations in $S_{\mathbf{q}}(V)$ is

$$
\wedge_{\mathbf{q}}^{2} V=\operatorname{span}\left(x_{i} \otimes x_{j}-q_{i j} x_{j} \otimes x_{i} \mid 1 \leq i, j \leq n\right) \quad \subset V \otimes V,
$$

the q-exterior square of $V$. For future use, we denote by $y_{1}, \ldots, y_{n}$ the basis of $V^{*}$ dual to $\left\{x_{i}\right\}$, so that $V^{*}=\operatorname{span}\left(y_{1}, \ldots, y_{n}\right)$. Furthermore, we introduce the Abelian group

$$
\Gamma_{\mathbf{q}} \subset \mathrm{GL}(V), \quad \Gamma_{\mathbf{q}}=\left\langle\gamma_{1}, \ldots, \gamma_{n}\right\rangle, \quad \gamma_{i}\left(x_{j}\right)=q_{i j} x_{j},
$$

and observe that $V$ is a Yetter-Drinfeld module over $\Gamma_{\mathbf{q}}$, via the natural action of $\Gamma_{\mathbf{q}}$ and the grading

$$
\left|x_{i}\right|=\gamma_{i}
$$


This gives rise to the braiding $\tau_{\mathbf{q}}$ on $V$, defined by $\tau_{\mathbf{q}}\left(x_{i} \otimes x_{j}\right)=q_{i j} x_{j} \otimes x_{i}$. The algebra $S_{\mathbf{q}}(V)$ coincides with the braided symmetric algebra $S\left(V, \tau_{\mathbf{q}}\right)$.

2.2. The $\mathbf{q}$-Heisenberg double. Our next step is to introduce a $\mathbf{q}$-differential calculus via a q-analogue of the Weyl algebra $\mathcal{D}(V)$. We have two candidates for the role of such q-analogue. One is the Heisenberg quadratic double $A_{V}$, associated to $V$ as a Yetter-Drinfeld module over the group $\Gamma_{\mathbf{q}}$. The other candidate is the braided Weyl algebra $\underline{A}_{V}$ (Definition 1.26) of $V$, and this will be relevant for braided Cherednik algebras later. The two are related by braided reduction, as described in Section 1

In this Section we construct q-Cherednik algebras as deformations of the Heisenberg quadratic double $A_{V}$. The presentation of $A_{V}$ is given in

Proposition 2.1. Let $V$ be viewed as a Yetter-Drinfeld module over the group $\Gamma_{\mathbf{q}}$ as above. The Heisenberg quadratic double $A_{\mathbf{q}}:=A_{V}$ has the triangular decomposition

$$
A_{\mathbf{q}} \cong S_{\mathbf{q}}(V) \otimes \mathbb{C} \Gamma_{\mathbf{q}} \otimes S_{\mathbf{q}^{\top}}\left(V^{*}\right),
$$

where $\mathbf{q}^{\top}$ is the transpose of the matrix $\mathbf{q}$, so that $S_{\mathbf{q}^{\top}}\left(V^{*}\right)$ is generated by $V^{*}$ subject to relations $y_{i} y_{j}=q_{j i} y_{j} y_{i}$. The commutation relation between $x_{i}$ and $y_{j}$ is $y_{j} x_{i}-x_{i} y_{j}=\delta_{i, j} \gamma_{i} \in \mathbb{C} \Gamma_{\mathbf{q}}$.

Proof. The braiding $\tau_{\mathbf{q}}^{*}$ on $V^{*}$ is computed as $\tau_{\mathbf{q}}^{*}\left(y_{i} \otimes y_{j}\right)=q_{j i} y_{j} \otimes y_{i}$ (note the order of the indices). One thus obtains the relations $y_{i} y_{j}-q_{j i} y_{j} y_{i}$ in $S\left(V^{*}, \tau_{\mathbf{q}}^{*}\right)$ as the kernel of id $+\tau_{\mathbf{q}}^{*}$. The claim then follows from Proposition 1.20 and the definition of the map $\beta_{Y}$ in Section 1

It is now natural to look for a q-analogue of rational Cherednik algebras among quadratic doubles with triangular decomposition

$$
A \cong S_{\mathbf{q}}(V) \otimes \mathbb{C} W \otimes S_{\mathbf{q}^{\top}}\left(V^{*}\right),
$$

where $W$ is a subgroup of $\mathrm{GL}(V)$. Our next step is to determine what the group $W$ can be.

2.3. The subgroup of $\mathrm{GL}(V)$ that preserves $\wedge_{\mathbf{q}}^{2} V$ and $\wedge_{\mathbf{q}^{\top}}^{2} V^{*}$. Suppose that $W$ is a subgroup of $\mathrm{GL}(V)$ such that there exists a quadratic double of the form $S_{\mathbf{q}}(V) \otimes \mathbb{C} W \otimes S_{\mathbf{q}^{\top}}\left(V^{*}\right)$. By Theorem 1.8

$$
W\left(\wedge_{\mathbf{q}}^{2} V\right)=\wedge_{\mathbf{q}}^{2} V, \quad W\left(\wedge_{\mathbf{q}^{\top}}^{2} V^{*}\right)=\wedge_{\mathbf{q}^{\top}}^{2} V^{*} .
$$

In the case $q_{i j}=1 \forall i, j$ (the commutative case) these conditions are vacuous; but they are not so in general. The group $W$ must be a subgroup of

$$
N(\mathbf{q})=\left\{w \in \mathrm{GL}(V) \mid w\left(\wedge_{\mathbf{q}}^{2} V\right)=\wedge_{\mathbf{q}}^{2} V, w\left(\wedge_{\mathbf{q}^{\top}}^{2} V^{*}\right)=\wedge_{\mathbf{q}^{\top}}^{2} V^{*}\right\} .
$$

To describe $N(\mathbf{q})$, we denote

$$
\mathbb{S}(\mathbf{q})=\left\{\sigma \in \mathbb{S}_{n} \mid q_{\sigma(i) \sigma(j)}=q_{i j} \forall i, j\right\}
$$


and view $\mathbb{S}(\mathbf{q})$ as a subgroup of $\mathrm{GL}(V)$ acting on $V$ by permutations of the basis $\left\{x_{i}\right\}$. Recall the grading $V=\oplus_{\gamma \in \Gamma_{\mathbf{q}}} V_{\gamma}$ given by $\left|x_{i}\right|=\gamma_{i}$ and observe that the component $V_{\gamma_{i}}$ of $V$ is spanned by $\left\{x_{j} \mid q_{j k}=q_{i k}\right.$ for all indices $\left.k\right\}$. Denote

$$
L(\mathbf{q})=\left\{g \in \mathrm{GL}(V) \mid g\left(V_{\gamma}\right)=V_{\gamma} \text { for all } \gamma \in \Gamma_{\mathbf{q}}\right\} .
$$

Clearly, the group $\mathbb{S}(\mathbf{q})$ normalises $L(\mathbf{q})$, therefore $\mathbb{S}(\mathbf{q}) \cdot L(\mathbf{q})$ is a subgroup in $\mathrm{GL}(V)$.

Proposition 2.2. 1. $N(\mathbf{q})=\mathbb{S}(\mathbf{q}) \cdot L(\mathbf{q})$.

2. $N(\mathbf{q})$ is the stabiliser of the set $\left\{\gamma_{i} \mid i=1, \ldots, n\right\}$ in $\mathrm{GL}(V)$ and is the normaliser of $\Gamma_{\mathbf{q}}$ in $\mathrm{GL}(V)$.

The Proposition will follow from two elementary lemmas.

Lemma 2.3. Let $c_{i}, c_{i}^{\prime}(i=1, \ldots, n)$ and $q$ be scalars, and let $x=\sum_{i} c_{i} x_{i}, x^{\prime}=\sum_{i} c_{i}^{\prime} x_{i}$. Then $x \otimes x^{\prime}-q x^{\prime} \otimes x \in \wedge_{\mathbf{q}}^{2} V$ if and only if $\left(1-q q_{i j}\right) c_{i}^{\prime} c_{j}=\left(q-q_{i j}\right) c_{i} c_{j}^{\prime}$ for all indices $i, j$.

Proof. Recall that $\wedge_{\mathbf{q}}^{2} V=\operatorname{ker}\left(\mathrm{id}+\tau_{\mathbf{q}}\right)$. Applying id $+\tau_{\mathbf{q}}$ to $x \otimes x^{\prime}-q x^{\prime} \otimes x$ and equating the coefficient of $x_{j} \otimes x_{i}$ with zero gives the desired identity.

Lemma 2.4. Let $A$ be an endomorphism of $V$ acting by $A x_{i}=\sum_{j} A_{i}^{j} x_{j}$. Then $A$ preserves $\wedge_{\mathbf{q}}^{2} V$ and the adjoint $A^{*}$ of $A$ preserves $\wedge_{\mathbf{q}^{\top}}^{2} V^{*}$, if and only if

$$
\left(q_{k l}-q_{i j}\right) A_{k}^{i} A_{l}^{j}=0
$$

for all indices $i, j, k, l$.

Proof. The condition that $A$ preserves $\wedge_{\mathbf{q}}^{2} V$ is equivalent to $A x_{k} \otimes A x_{l}-q_{k l} A x_{l} \otimes A x_{k} \in \wedge_{\mathbf{q}}^{2} V$ for all $k, l$. By Lemma 2.3 this is the same as $\left(1-q_{k l} q_{i j}\right) A_{l}^{i} A_{k}^{j}=\left(q_{k l}-q_{i j}\right) A_{k}^{i} A_{l}^{j}$ for all $i, j, k, l$. The matrix of $A^{*}$ with respect to the basis $\left\{y_{i}\right\}$ dual to $\left\{x_{i}\right\}$ is the transpose of the matrix of $A$, and $\mathbf{q}^{\top}$ is the transpose of $\mathbf{q}$, therefore in $V^{*}$ we obtain the condition $\left(1-q_{l k} q_{j i}\right) A_{i}^{l} A_{j}^{k}=\left(q_{l k}-q_{j i}\right) A_{i}^{k} A_{j}^{l}$. Swapping the indices $i, l$, as well as $j, k$, we get $\left(1-q_{k l} q_{i j}\right) A_{l}^{i} A_{k}^{j}=-\left(q_{k l}-q_{i j}\right) A_{k}^{i} A_{l}^{j}$. Hence both sides of this equation are zero.

Proof of Proposition 2.2. 1. It is clear that $\mathbb{S}(\mathbf{q})$ and $L(\mathbf{q})$ both preserve $\wedge_{\mathbf{q}}^{2} V$ and $\wedge_{\mathbf{q}^{\top}}^{2} V^{*}$, thus it is enough to show that $N(\mathbf{q}) \subset \mathbb{S}(\mathbf{q}) \cdot L(\mathbf{q})$. Let $w \in N(\mathbf{q})$. By definition of the action of $\mathrm{GL}(V)$ on $V^{*}$, the action of $w^{-1}$ on $V^{*}$ is by the adjoint $w^{*}$ of $w$, thus $w^{*}$ preserves $\wedge_{\mathbf{q}^{\top}}^{2} V^{*}$. By Lemma 2.4 $\left(q_{k l}-q_{i j}\right) w_{k}^{i} w_{l}^{j}=0$ for all indices $i, j, k, l$, where $w_{k}^{i}$ are entries of the matrix of $w$ in the basis $\left\{x_{i}\right\}$. By invertibility of $w$, there exists a permutation $\sigma \in \mathbb{S}_{n}$ such that $w_{i}^{\sigma(i)} \neq 0$ for all indices $i$. For any pair $i, j$ of indices one has the relation $\left(q_{i j}-q_{\sigma(i) \sigma(j)}\right) w_{i}^{\sigma(i)} w_{j}^{\sigma(j)}=0$, hence $q_{i j}=q_{\sigma(i) \sigma(j)}$ and $\sigma \in \mathbb{S}(\mathbf{q})$. 
We are left to prove that the matrix $g:=\sigma^{-1} w$, with entries also satisfying the equation in Lemma 2.4 and with $g_{i}^{i} \neq 0$ for all $i$, is in $L(\mathbf{q})$; equivalently, that $\gamma_{i} \neq \gamma_{j} \in \Gamma_{\mathbf{q}}$ implies $g_{i}^{j}=0$. Indeed, find $l$ such that $q_{i l} \neq q_{j l}$. The relation $\left(q_{i l}-q_{j l}\right) g_{i}^{j} g_{l}^{l}=0$ implies that $g_{i}^{j}=0$ as required.

2. For $g \in \mathrm{GL}(V), g \Gamma_{\mathbf{q}} g^{-1}=\Gamma_{\mathbf{q}}$ if and only if $g$ permutes the simultaneous eigenspaces of $\Gamma_{\mathbf{q}}$. These are the same as simultaneous eigenspaces of $\gamma_{i}$, i.e., the subspaces $V_{\gamma_{j}}$ of $V$. It is obvious that such $g$ are precisely elements of $\mathbb{S}(\mathbf{q}) L(\mathbf{q})$.

Corollary 2.5. Let $W$ be a subgroup of $\mathrm{GL}(V)$ that contains $\Gamma_{\mathbf{q}}$. The group $W$ preserves $\wedge_{\mathbf{q}}^{2} V$ and $\wedge_{\mathbf{q}^{\top}}^{2} V^{*}$ if and only if $V$ is a Yetter-Drinfeld module via the $W$-action on $V$ and the $W$-grading by $\left|x_{i}\right|=\gamma_{i}$.

Remark 2.6. An element $w \in \mathrm{GL}(V)$ stabilises $\wedge_{\mathbf{q}^{\top}}^{2} V^{*}$, if and only if $w$ stabilises the $\mathbf{q}$-symmetric square $S_{\mathbf{q}}^{2}(V):=\operatorname{span}_{i, j}\left(x_{i} \otimes x_{j}+q_{i j} x_{j} \otimes x_{i}\right)$ of $V$. This is because $S_{\mathbf{q}}^{2}(V)$ is the orthogonal complement of $\wedge_{\mathbf{q}^{\top}}^{2} V^{*}$ with respect to the standard pairing. Note that $S_{\mathbf{q}}^{2}(V), \wedge_{\mathbf{q}}^{2} V$ are the eigenspaces of the involutive braiding $\tau_{\mathbf{q}}$ on $V \otimes V$. Therefore, $N(\mathbf{q})$ is the centraliser of $\tau_{\mathbf{q}}$ in $\operatorname{GL}(V)$.

2.4. q-Cherednik algebras. To obtain a nice classification of deformations of the Heisenberg quadratic double $A_{\mathbf{q}}$, we impose an extra nondegeneracy condition:

Definition 2.7. A quadratic double $A \cong T(V) /<R^{-}>\otimes \mathbb{C} W \otimes T\left(V^{*}\right) /<R^{+}>$is called non-degenerate, if the commutator map $[\cdot, \cdot]: V^{*} \times V \rightarrow \mathbb{C} W$ has no non-trivial kernels in $V^{*}$ and in $V$.

Definition 2.8. A q-Cherednik algebra is a non-degenerate quadratic double with triangular decomposition $S_{\mathbf{q}}(V) \otimes \mathbb{C} W \otimes S_{\mathbf{q}^{\top}}\left(V^{*}\right)$, where $W$ is a subgroup of GL(V) (not necessarily finite).

In the next Proposition, we keep the notation for $\mathbf{q}, x_{i}, y_{i}, V$ and $W$.

Proposition 2.9. A q-Cherednik algebra is generated by $x_{1}, \ldots, x_{n} \in V, w \in W$ and $y_{1}, \ldots, y_{n} \in$ $V^{*}$ subject to relations

- $x_{i} x_{j}=q_{i j} x_{j} x_{i}, \quad y_{i} y_{j}=q_{j i} y_{j} y_{i}, \quad w x_{i} w^{-1}=w\left(x_{i}\right) \in V, \quad w y_{i} w^{-1}=w\left(y_{i}\right) \in V^{*}$, and

- $y_{j} x_{i}-x_{i} y_{j}=\sum_{w \in W}\left\langle L_{w}\left(x_{i}\right), y_{j}\right\rangle w$ for some $L_{w} \in \operatorname{End}(V)$.

The maps $L_{w}$ are such that $\cap_{w \in W} \operatorname{ker} L_{w}=0, \cap_{w \in W} \operatorname{ker} L_{w}^{*}=0$, and satisfy

$$
\begin{array}{r}
\left.g\left(L_{w}\left(g^{-1}(x)\right)\right)=L_{g w g^{-1}}(x) \quad \text { (W-equivariance }\right) \\
\left(x_{i}-q_{i j} w\left(x_{i}\right)\right) \otimes L_{w}\left(x_{j}\right)=\left(q_{i j} x_{j}-w\left(x_{j}\right)\right) \otimes L_{w}\left(x_{i}\right) \\
\left(y_{i}-q_{j i} w\left(y_{i}\right)\right) \otimes L_{w}^{*}\left(y_{j}\right)=\left(q_{j i} y_{j}-w\left(y_{j}\right)\right) \otimes L_{w}^{*}\left(y_{i}\right) \quad \text { (q-commutativity equations) }
\end{array}
$$

for all $g, w \in W$ and all indices $i, j$. Conversely, an algebra with the above presentation, with $W \leq \mathrm{GL}(V)$ centralising $\tau_{\mathbf{q}}$ and $L_{w}$ subject to the above conditions, is a $\mathbf{q}$-Cherednik algebra. 
Proof. The defining relations follow from the definition of a $\mathbf{q}$-Cherednik algebra as a quadratic double, while $\cap_{w \in W} \operatorname{ker} L_{w}=0, \cap_{w \in W}$ ker $L_{w}^{*}=0$ is precisely the nondegeneracy condition. Furthermore, the $W$-equivariance condition in the Proposition is the same as $W$-equivariance of the commutator, as required by Theorem 1.4. It remains to show that the q-commutativity equations are equivalent to the conditions $\left[R^{+}, V\right]=0$ and $\left[V^{*}, R^{-}\right]=0$ in Theorem 1.8 , where $R^{+}=\wedge_{\mathbf{q}}^{2} V^{*}$ and $R^{-}=\wedge_{\mathbf{q}}^{2} V$. To analyse the commutator $\left[V^{*}, R^{-}\right]$, write

$$
\begin{aligned}
{\left[y, x_{i} x_{j}-q_{i j} x_{j} x_{i}\right] } & =x_{i}\left[y, x_{j}\right]-q_{i j}\left[y, x_{j}\right] x_{i}+\left[y, x_{i}\right] x_{j}-q_{i j} x_{j}\left[y, x_{i}\right] \\
& \left.=\sum_{w \in W}\left(x_{i} w-q_{i j} w x_{i}\right)\left\langle L_{w}\left(x_{j}\right), y\right\rangle-\left(q_{i j} x_{j} w-w x_{j}\right)\left\langle L_{w}\left(x_{i}\right)\right), y\right\rangle \\
& \left.\left.=\sum_{w \in W}\left(x_{i}-q_{i j} w\left(x_{i}\right)\right) \cdot w \cdot\left\langle L_{w}\left(x_{j}\right), y\right\rangle-\left(q_{i j} x_{j}-w\left(x_{j}\right)\right)\right) \cdot w \cdot\left\langle L_{w}\left(x_{i}\right)\right), y\right\rangle,
\end{aligned}
$$

which vanishes for all $y \in V^{*}$ if and only if the first q-commutativity equation holds. Similarly, $\left[R^{+}, V\right]=0$ is equivalent to the second $\mathbf{q}$-commutativity equation.

2.5. The block structure of the matrix q. The structure of the subgroup $\mathbb{S}(\mathbf{q})$ of $\mathbb{S}_{n}$ and its action on the space $V$ may be complicated, depending on the combinatorics of the matrix q. We will soon show, however, that the $\mathbf{q}$-commutativity equations in Proposition 2.9 imply that only the part of $\mathbb{S}(\mathbf{q})$ generated by transpositions actually matters for $\mathbf{q}$-Cherednik algebras. This leads to $V$ and the matrix $\mathbf{q}$ being split into blocks; let us formally introduce this block structure.

Definition 2.10. We say that indices $i, j \in\{1, \ldots, n\}$ are in the same block (with respect to the matrix $\mathbf{q})$, if

$$
q_{i k}=q_{j k} \quad \text { for all } k \neq i, j ; \quad q_{i j}= \pm 1 .
$$

Lemma 2.11. Being in the same block is an equivalence relation on the set $\{1, \ldots, n\}$ of indices. An equivalence class $B$ (a block of indices) can be of one of the following two types:

- positive block: $q_{i j}=1$ for all $i, j \in B$;

- negative block: $q_{i j}=-1$ for all $i, j \in B, i \neq j$, where $|B|>1$.

Proof. Let us write $i \sim_{+} j$, respectively $i \sim_{-} j$, if $i, j$ are indices such that $q_{i k}=q_{j k}$ for any $k \neq i, j$ and $q_{i j}=1$ (respectively $q_{i j}=-1$ ). We need to check that the relation $\sim=_{+} \cup \sim_{-}$is an equivalence relation. Note that $\sim_{+}$is an equivalence relation, because $i \sim_{+} j$ means that rows $i$ and $j$ of the matrix $\mathbf{q}$ are identical. Hence $\sim$ is reflexive, and is symmetric as both $\sim_{+}$and $\sim_{-}$are. Moreover, $\sim_{+}$is transitive, therefore it remains to check that $a \sim_{-} b \sim c$ implies $a \sim c$. If $c=a$ or $c=b$, we are done, otherwise $a \sim_{-} b$ implies $q_{a c}=q_{b c}$ and $b \sim c$ implies $q_{b a}=q_{c a}$. Since $q_{b a}=-1$, we have $q_{a c}=q_{b c}=-1$ and $q_{a b}=q_{c b}=-1$. Finally, for any $k \neq a, b, c$ we have $q_{a k}=q_{b k}=q_{c k}$. Thus, $a \sim_{-} c$. 
Corollary 2.12 (Block structure of the matrix $\mathbf{q}$ ). Let the matrix $\mathbf{q}$ be given. The index set $\{1, \ldots, n\}$ is split into disjoint blocks. To each pair $B, C$ of blocks there is associated a complex number $q_{B, C}=q_{C, B}^{-1} \in \mathbb{C}$ such that

$$
q_{i j}=q_{B, C} \quad \text { whenever } i \neq j, \quad i \in B, j \in C .
$$

In particular, $q_{B, B}$ is 1 or -1 , depending on whether the block $B$ is positive or negative.

Let $B$ be a block of indices. Introduce the following subspaces:

$$
V_{B}=\operatorname{span}\left(x_{i} \mid i \in B\right) \quad \subset V, \quad V_{B}^{*}=\operatorname{span}\left(y_{i} \mid i \in B\right) \quad \subset V^{*},
$$

and let $\gamma_{B} \in N(\mathbf{q}) \subset \mathrm{GL}(V)$ be such that

$$
\left.\gamma_{B}\right|_{V_{C}}=q_{B, C} \operatorname{id}_{V_{C}}
$$

for any block $C$, where the scalars $q_{B, C}$ are as introduced in Corollary 2.12 .

2.6. q-Cherednik algebras: the structure theorem. Proposition 2.9 gives the relations in a q-Cherednik algebra explicitly, except the most important one - the commutation relation between $V^{*}$ and $V$. It turns out that, similar to rational Cherednik algebras, the commutator is expressed in terms of complex reflections in the group $W$, but premultiplied with elements $\gamma_{B}$ as an extra ingredient. For reference, we need a list of complex reflections in $\mathrm{GL}(V)$ that preserve the relations in the algebras $S_{\mathbf{q}}(V)$ and $S_{\mathbf{q}^{\top}}\left(V^{*}\right)$.

Lemma 2.13. Let $s \in \mathrm{GL}(V)$ be a complex reflection (not necessarily of finite order) and $\alpha_{s} \in V$, $\alpha_{s}^{\vee} \in V^{*}$ be the root-coroot pair for $s$. If $s \in N(\mathbf{q})$, then:

(1) There is a block $B \subset\{1, \ldots, n\}$ of indices, such that $\alpha_{s} \in V_{B}$ and $\alpha_{s}^{\vee} \in V_{B}^{*}$.

(2) If $B$ is a positive block, $s$ is an arbitrary complex reflection in the space $V_{B}$.

(3) If $B$ is negative, $s$ must be of the form $t_{i}^{(\eta)}(\eta \neq 0,1)$ or $(i j) t_{i}^{(\varepsilon)} t_{j}^{\left(\varepsilon^{-1}\right)}$. Here $(i j)$ permutes variables $x_{i}$ and $x_{j}$ with $i, j \in B$, and $t_{i}^{(\varepsilon)}$ multiplies the variable $x_{i}$ by $\varepsilon \in \mathbb{C}^{\times}$, leaving the rest of the variables intact.

Proof. (1) By Proposition 2.2 $s=\bar{s} \cdot g$ where $\bar{s}$ is a permutation of indices such that $q_{\bar{s}(i) \bar{s}(j)}=q_{i j}$, and $g$ preserves all $\Gamma_{\mathbf{q}}$-graded components $V_{\gamma_{k}}$ of $V$. It follows that $(1-s) V_{\gamma_{k}} \subset V_{\gamma_{k}}+V_{\gamma_{\bar{s}(k)}}$. But then $\operatorname{dim}(1-s) V=1$ implies that there are at most two indices $k$ such that $(1-s) V_{\gamma_{k}} \neq 0$. If there is only one such index $k$, let $B$ be the block of indices containing $k$. Otherwise, there are two such indices $i, j$, and necessarily $\bar{s}$ is the permutation $(i j)$. One has $q_{j i}=q_{i j}$ (hence $q_{i j}= \pm 1$ ) and $q_{i a}=q_{j a}$ for all $a \neq i, j$, thus $i, j$ belong to the same block; let $B$ be the block which contains $i, j$. In either case, $(1-s) V_{B^{\prime}}=0$ for $B^{\prime} \neq B$ and $(1-s) V_{B} \subset V_{B}$, which implies $\alpha_{s} \in V_{B}$ and $\alpha_{s}^{\vee} \in V_{B}^{*}$. 
Part (2) is clear, as $V_{B}$ is of the form $V_{\gamma_{k}}$ if $B$ is a positive block, thus any complex reflection $s$ in $V_{B}$ has decomposition id $\cdot s \in \mathbb{S}(\mathbf{q}) \cdot L(\mathbf{q})$ and hence commutes with $\tau_{\mathbf{q}}$.

Finally, if $B$ is a negative block, then by Lemma $2.11 V_{B}=\oplus\left\{V_{\gamma_{i}} \mid i \in B\right\}$ is a direct sum of one-dimensional $\Gamma_{\mathbf{q}}$-graded components. By Proposition 2.2 $s$ must act imprimitively and permute these 1-dimensional subspaces. All such imprimitive complex reflections are listed in (3), cf. [DO, Section 3].

The following Theorem completes the description of the structure of q-Cherednik algebras.

Theorem 2.14. Let $A \cong S_{\mathbf{q}}(V) \otimes \mathbb{C} W \otimes S_{\mathbf{q}^{\top}}\left(V^{*}\right)$ be a $\mathbf{q}$-Cherednik algebra. Then the commutator of $y \in V^{*}$ and $x \in V$ in $A$ is of the form

$$
y x-x y=\sum_{\text {blocks } B} \gamma_{B} \cdot\left((x, y)_{B} \cdot 1+\sum_{s} c_{s}\left\langle x, \alpha_{s}^{\vee}\right\rangle\left\langle\alpha_{s}, y\right\rangle s\right),
$$

where the sum is taken over complex reflections $s$ which commute with the braiding $\tau_{\mathbf{q}}$ and such that $\alpha_{s} \in V_{B}, \alpha_{s}^{\vee} \in V_{B}^{*}$ and $\gamma_{B} s \in W$. The pairing $(\cdot, \cdot)_{B}$ between $V$ and $V^{*}$ is such that $\left(x_{i}, y_{j}\right)_{B}=0$ unless $i, j \in B$, and is so chosen, together with the constants $c_{s}$, as to make the commutator $W$-equivariant and non-degenerate.

Corollary 2.15. In particular, in a $\mathbf{q}$-Cherednik algebra one has $y_{j} x_{i}-x_{i} y_{j}=0$ unless $i, j$ are in the same block of indices with respect to $\mathbf{q}$.

Proof of the Theorem. We write the commutator as $[y, x]=\sum_{w \in W}\left\langle L_{w}(x), y\right\rangle w$ with $L_{w} \in \operatorname{End}(V)$. It is enough to show that if the map $L_{w}$ is non-zero, then either

(a) $w=\gamma_{B}$ for some block $B, L_{w}\left(V_{B^{\prime}}\right)=0$ for blocks $B^{\prime} \neq B$, and $L_{w}\left(V_{B}\right) \subset V_{B}$; or

(b) $w=\gamma_{B} s$ for a complex reflection $s$ such that $\alpha_{s} \in V_{B}, \alpha_{s}^{\vee} \in V_{B}^{*}$, and $L_{w}(x)=$ const. $\left\langle x, \alpha_{s}^{\vee}\right\rangle \alpha_{s}$.

Case 1: $w$ preserves each $\Gamma_{\mathbf{q}}$-homogeneous component $V_{\gamma_{i}}$ of $V$. Find an index $i$ such that $L_{w}\left(x_{i}\right) \neq 0$. For an index $j$ such that $\gamma_{j} \neq \gamma_{i}$, the vectors $x_{i}-q_{i j} w\left(x_{i}\right) \in V_{\gamma_{i}}$ and $q_{i j} x_{j}-w\left(x_{j}\right) \in$ $V_{\gamma_{j}}$ cannot be nonzero and proportional, therefore, both sides of the q-commutativity equation in Proposition 2.9 must be zero. It follows that $q_{i j} x_{j}-w\left(x_{j}\right)=L_{w}\left(x_{j}\right)=0$. Hence $\left.w\right|_{V_{\gamma_{j}}}=\left.\gamma_{i}\right|_{V_{\gamma_{j}}}$ and $\left.L_{w}\right|_{V_{\gamma_{j}}}=0$ on all $\Gamma_{\mathbf{q}}$-homogeneous components $V_{\gamma_{j}}$ of $V$ such that $\gamma_{j} \neq \gamma_{i}$. Similarly, $L_{w}^{*}$ vanishes on $\Gamma_{\mathbf{q}}$-homogeneous components of $V^{*}$ other than $\left(V^{*}\right)_{\gamma_{i}^{-1}}$, which means that $L_{w}\left(V_{\gamma_{i}}\right) \subset V_{\gamma_{i}}$.

Furthermore, Proposition 2.9 implies that for $x, x^{\prime} \in V_{\gamma_{i}}$

$$
(x-w(x)) \otimes L_{w}\left(x^{\prime}\right)=\left(x^{\prime}-w\left(x^{\prime}\right)\right) \otimes L_{w}(x) .
$$

It is easy to see that this tensor equation may hold only either if $w=\operatorname{id}$ on $V_{\gamma_{i}}$, or if $\operatorname{dim}(1-w) V_{\gamma_{i}}=$ $\operatorname{dim} L_{w}\left(V_{\gamma_{i}}\right)=1$. In the former case, $w=\gamma_{i}$. If $i$ belongs to a positive block $B$, one has $V_{B}=V_{\gamma_{i}}$ and 
therefore $w=\gamma_{B}$, so that option (a) holds. If $i$ belongs to a negative block $B$, then $\operatorname{dim} V_{\gamma_{i}}=1$, therefore $L_{w}(x)=$ const $\cdot\left\langle x, y_{i}\right\rangle x_{i}$. The element $w=\gamma_{i}$ decomposes as $\gamma_{B} t_{i}^{(-1)}$, and $t_{i}^{(-1)}$ is a complex reflection on $V_{\gamma_{i}}=\mathbb{C} x_{i}$ with root-coroot pair $x_{i}, 2 y_{i}$, so that option (b) holds.

In the case $\operatorname{dim}(1-w) V_{\gamma_{i}}=\operatorname{dim} L_{w}\left(V_{\gamma_{i}}\right)=1$, the element $w$ is necessarily $\gamma_{i} s$, where $s$ is a complex reflection on $V_{\gamma_{i}}$. Write $L_{w}(x)=\langle x, \alpha\rangle \beta$ with $\alpha \in V_{\gamma_{i}^{-1}}^{*}$ and $\beta \in V_{\gamma_{i}}$. By (図), $L_{w}(x)$ vanishes on $\operatorname{ker}(1-s)$, therefore $\alpha=$ const $\cdot \alpha_{s}^{\vee}$. Moreover, Proposition 2.9 implies the equation $(y-s(y)) \otimes L_{w}^{*}\left(y^{\prime}\right)=\left(y^{\prime}-s\left(y^{\prime}\right)\right) \otimes L_{w}^{*}(y)$ for $y, y^{\prime} \in\left(V^{*}\right)_{\gamma_{i}^{-1}}$, so that $\beta=$ const $\cdot \alpha_{s}$. If $i$ belongs to a positive block $B$, we have $V_{B}=V_{\gamma_{i}}$, and option $(a)$ holds. If $i$ belongs to a negative block $B$ so that $\operatorname{dim} V_{\gamma_{i}}=1$, then $s=t_{i}^{(\eta)}$ for some $\eta \neq 1$, and $w=\gamma_{i} t_{i}^{(\eta)}=\gamma_{B} t_{i}^{(-\eta)}$. No matter what $\eta$ is, the root and the coroot of $t_{i}^{(-\eta)}$ are proportional to $x_{i}$ and $y_{i}$, respectively, hence option $(b)$ still holds.

Case 2: there exist indices $i, j$ such that $\gamma_{i} \neq \gamma_{j}$ and $w\left(V_{\gamma_{i}}\right)=V_{\gamma_{j}}$. By Proposition 2.9,

$$
\left(x_{i}-q_{i k} w\left(x_{i}\right)\right) \otimes L_{w}\left(x_{k}\right)=\left(q_{i k} x_{k}-w\left(x_{k}\right)\right) \otimes L_{w}\left(x_{i}\right) \quad \text { for all } k .
$$

Note that $x_{i}-q_{i k} w\left(x_{i}\right)$ cannot be zero, because $x_{i} \in V_{\gamma_{i}}, w\left(x_{i}\right) \in V_{\gamma_{j}}$ and $V_{\gamma_{i}} \cap V_{\gamma_{j}}=0$. Therefore $L_{w}\left(x_{i}\right) \neq 0$, as otherwise the commutativity equation would imply that $L_{w}\left(x_{k}\right)=0$ for all $k$. Now observe that for any $x \in V_{\gamma_{j}}, x \neq 0$ one has

$$
\left(x_{i}-q_{i j} w\left(x_{i}\right)\right) \otimes L_{w}(x)=\left(q_{i j} x-w(x)\right) \otimes L_{w}\left(x_{i}\right)
$$

and $q_{i j} x-w(x) \neq 0$ because $w\left(V_{\gamma_{j}}\right) \cap V_{\gamma_{j}}=0$. It follows that $L_{w}(x)$ is proportional to $L_{w}\left(x_{i}\right)$ for any $x \in V_{\gamma_{j}}$, thus $\operatorname{dim} L_{w}\left(V_{\gamma_{j}}\right)=1$.

Now if the $\operatorname{dim} V_{\gamma_{j}}$ is greater than 1, the map $L_{w}$ must have a kernel in $V_{\gamma_{j}}$. Pick $0 \neq x \in V_{\gamma_{j}}$ such that $L_{w}(x)=0$. Substituting $x$ in (买) leads to a contradiction, as the left-hand side of the equation becomes zero while the right-hand side does not. Therefore $\operatorname{dim} V_{\gamma_{i}}=\operatorname{dim} V_{\gamma_{j}}=1$ and $w\left(x_{i}\right)=\varepsilon x_{j}$ for some $\varepsilon \in \mathbb{C}^{\times}$.

By (买), $x_{i}-q_{i j} \varepsilon x_{j}$ is proportional to $q_{i j} x_{j}-w\left(x_{j}\right)$. It follows that $w\left(x_{j}\right)=\varepsilon^{-1} x_{i}$. Hence for $l \neq i, j$ the subspace $w\left(V_{\gamma_{l}}\right)$ has zero intersection with $V_{\gamma_{i}}$ and with $V_{\gamma_{j}}$, thus the vector $q_{i l} x_{l}-w\left(x_{l}\right)$ cannot coincide with $x_{i}-q_{i l} w\left(x_{i}\right)$ up to a non-zero factor. Equation (1**) therefore forces $q_{i l} x_{l}-$ $w\left(x_{l}\right)=0$ and $L_{w}\left(x_{l}\right)=0$ for each $l \neq i, j$. A similar equation in $V^{*}$ forces $L_{w}^{*}\left(y_{l}\right)=0$ for $l \neq i, j$, hence $L_{w}\left(\mathbb{C} x_{i}+\mathbb{C} x_{j}\right) \subset \mathbb{C} x_{i}+\mathbb{C} x_{j}$.

Furthermore, Proposition 2.2 implies that $q_{i k}=q_{k i}$ and that $q_{i l}=q_{j l}$ for all $l \neq i, j$. As $\gamma_{i} \neq \gamma_{j}$, one has $q_{i j}=-1$. This means that $i$ and $j$ belong to a negative block $B$, and $w$ acts as $\gamma_{B}$ on each $V_{\gamma_{l}}$ with $l \neq i, j$. We have the decomposition

$$
w=\gamma_{B} \cdot(i j) t_{i}^{(\varepsilon)} t_{j}^{\left(\varepsilon^{-1}\right)}
$$


Now (**) reads

$$
\left(x_{i}+\varepsilon x_{j}\right) \otimes L_{w}\left(x_{j}\right)=-\left(\varepsilon^{-1} x_{i}+x_{j}\right) \otimes L_{w}\left(x_{i}\right),
$$

so that $L_{w}\left(x_{i}\right)=-\varepsilon L_{w}\left(x_{j}\right)$. Moreover, the $W$-equivariance condition in Proposition 2.9 implies that $L_{w}$ commutes with $w$, whence $w\left(L_{w}\left(x_{i}\right)\right)=\varepsilon L_{w}\left(x_{j}\right)=-L_{w}\left(x_{i}\right)$, thus $L_{w}\left(x_{i}\right)=x_{i}-\varepsilon x_{j}$. It follows that

$$
L_{w}(x)=\text { const } \cdot\left\langle x, y_{i}-\varepsilon^{-1} y_{j}\right\rangle\left(x_{i}-\varepsilon x_{j}\right)
$$

and option (b) holds.

Corollary 2.16. Let $W$ be a subgroup of $\mathrm{GL}(V)$ centralising the braiding $\tau_{\mathbf{q}}$. An algebra given by generators and relations from Proposition 2.9 and the commutation relation from Theorem 2.14 is a q-Cherednik algebra, if the commutator is $W$-equivariant and non-degenerate.

Proof. Indeed, we checked in the proof of Theorem 2.14 that the q-commutativity equations in Proposition 2.9 are satisfied.

\section{BRAIDED CHEREDNIK ALGEBRAS}

In this Section, we introduce braided Cherednik algebras. Besides the well-known rational Cherednik algebras of Etingof and Ginzburg, irreducible groups give rise to a new class of negative braided Cherednik algebras.

3.1. The q-Weyl algebra $\underline{A}_{\mathbf{q}}$. Recall from Section 2 that the classical Weyl algebra of polynomial differential operators on the space $V$ admits two possible q-versions. One of them is the Heisenberg quadratic double $A_{\mathbf{q}} \cong S_{\mathbf{q}}(V) \otimes \mathbb{C} \Gamma_{\mathbf{q}} \otimes S_{\mathbf{q}^{\top}}\left(V^{*}\right)$ over the group $\Gamma_{\mathbf{q}}$; we introduced q-Cherednik algebras as deformations of this. The other is the braided Weyl algebra of $V$, obtained from $A_{\mathbf{q}}$ via braided reduction. We denote it by $\underline{A}_{\mathbf{q}}$ and will now review it in more detail. Note the appearance of the q-symmetric algebra

$$
S_{\mathbf{q}}\left(V^{*}\right):=\mathbb{C}\left\langle\underline{y}_{1}, \ldots, \underline{y}_{n} \mid \underline{y}_{i} \underline{y}_{j}=q_{i j} \underline{y}_{j} \underline{y}_{i}\right\rangle,
$$

which is not the same as $S_{\mathbf{q}^{\top}}\left(V^{*}\right)$ used in the previous Section; in fact, $S_{\mathbf{q}}\left(V^{*}\right) \cong S_{\mathbf{q}^{\top}}\left(V^{*}\right)^{\mathrm{op}}$.

Proposition 3.1. Let $V$ be viewed as a Yetter-Drinfeld module over the group $\Gamma_{\mathbf{q}}$ as above. The braided Weyl algebra $\underline{A}_{\mathbf{q}}:=\underline{A}_{V}$ decomposes as

$$
\underline{A}_{\mathbf{q}} \cong S_{\mathbf{q}}(V) \otimes S_{\mathbf{q}}\left(V^{*}\right),
$$

where $\underline{y}_{1} \ldots, \underline{y}_{n}$ are a basis of $V^{*}$ dual to $\left\{x_{i}\right\}$, and the commutation relation between $\underline{y}_{j}$ and $x_{i}$ is given by

$$
\underline{y}_{j} x_{i}-q_{i j} x_{i} \underline{y}_{j}=\delta_{i, j} .
$$


Proof. Follows immediately from Proposition 2.1 and Proposition 1.22. Alternatively, can be deduced from Example 1.25 .

We can view the space $V \oplus V^{*}$ as a Yetter-Drinfeld module over the group $\Gamma_{\mathbf{q}}$ (a direct sum of two YD modules) and denote the resulting braiding on $V \oplus V^{*}$ again by $\tau_{\mathbf{q}}$. Then one has the braided commutator

$$
[a, b]_{\mathbf{q}}:=a \otimes b-\tau_{\mathbf{q}}(a \otimes b), \quad a, b \in V \oplus V^{*} .
$$

The $\Gamma_{\mathbf{q}}$-grading on $V \oplus V^{*}$ is given by $\left|x_{i}\right|=\gamma_{i},\left|\underline{y}_{i}\right|=\gamma_{i}^{-1}$, and recall that $\gamma_{i}\left(x_{j}\right)=q_{i j} x_{j}$, $\gamma_{i}\left(\underline{y}_{j}\right)=q_{j i} \underline{y}_{j}$. Hence, the $\mathbf{q}$-commutator is explicitly written as

$\left[x_{i}, x_{j}\right]_{\mathbf{q}}=x_{i} \otimes x_{j}-q_{i j} x_{j} \otimes x_{i}, \quad\left[\underline{y}_{i}, \underline{y}_{j}\right]_{\mathbf{q}}=\underline{y}_{i} \otimes \underline{y}_{j}-q_{i j} \underline{y}_{j} \otimes \underline{y}_{i}, \quad\left[\underline{y}_{j}, x_{i}\right]_{\mathbf{q}}=\underline{y}_{j} \otimes x_{i}-q_{i j} x_{i} \otimes \underline{y}_{j}$.

Let $\omega(a, b)$ be the skew-symmetric bilinear form on $V \oplus V^{*}$ uniquely determined by $\omega\left(x, x^{\prime}\right)=$ $\omega\left(y, y^{\prime}\right)=0, \omega(x, y)=\langle x, y\rangle$ for $x, x^{\prime} \in V, y, y^{\prime} \in V^{*}$. The q-Weyl algebra can be defined as

$$
\underline{A}_{\mathbf{q}}=T\left(V \oplus V^{*}\right) /<[a, b]_{\mathbf{q}}-\omega(a, b)>.
$$

Moreover, any subgroup $W \leq \mathrm{GL}(V)$ which preserves the q-deformed exterior squares $\wedge_{\mathbf{q}}^{2} V$ and $\wedge_{\mathbf{q}^{\top}}^{2} V^{*}$, will also preserve $\wedge_{\mathbf{q}}^{2} V^{*}=\tau\left(\wedge_{\mathbf{q}^{\top}}^{2} V^{*}\right)$ and centralise the braiding $\tau_{\mathbf{q}}$ on $V \oplus V^{*}$. Trivially, $W$ preserves the form $\omega$, therefore the $\mathbf{q}$-Weyl algebra $\underline{A}_{\mathbf{q}}$ will be a $W$-module algebra.

3.2. Braided Cherednik algebras. Informally, one can now interpret $\omega$ in the above presentation of the braided Weyl algebra $\underline{A}_{\mathbf{q}}$ as a $\mathbb{C} W$-valued form. This leads to a braided version of Drinfeld's degenerate affine Hecke algebra $[\mathrm{Dr}$ ] and Etingof-Ginzburg symplectic reflection algebra [EG], and is a natural way to introduce braided Cherednik algebras. Their formal definition is as follows.

Definition 3.2. A braided Cherednik algebra associated to the matrix $\mathbf{q}$ is an algebra with triangular decomposition $S_{\mathbf{q}}(V) \otimes \mathbb{C} W \otimes S_{\mathbf{q}}\left(V^{*}\right)$ where

$$
w x w^{-1}=w(x), \quad w y w^{-1}=w(y), \quad[y, x]_{\mathbf{q}} \in \mathbb{C} W
$$

for $x \in V, y \in V^{*}, w \in W$, such that the braided commutator $[\cdot, \cdot]_{\mathbf{q}}: V^{*} \times V \rightarrow \mathbb{C} W$ has zero kernels in $V^{*}$ or $V$.

To establish the connection to q-Cherednik algebras, recall that if there exists a q-Cherednik algebra of a group $\widetilde{W} \leq \mathrm{GL}(V)$ such that $\widetilde{W}$ contains $\Gamma_{\mathbf{q}}$, then $V$ is a Yetter-Drinfeld module over $W$ by Corollary 2.5. This means that such q-Cherednik algebra has braided reduction. We have

Proposition 3.3. Braided Cherednik algebras associated to the matrix $\mathbf{q}$ are the same as braided reductions of $\mathbf{q}$-Cherednik algebras. 
Proof. First, assume that $A=S_{\mathbf{q}}(V) \otimes \mathbb{C} \widetilde{W} \otimes S_{\mathbf{q}^{\top}}\left(V^{*}\right)$ is a q-Cherednik algebra where $\widetilde{W} \supset \Gamma_{\mathbf{q}}$. Then it is easily deduced from Proposition 1.22 that $\underline{A}$, which is the same algebra as $A$ but with generators $x_{i}, w$ and

$$
\underline{y}_{i}=\gamma_{i}^{-1} y_{i}
$$

has triangular decomposition $S_{\mathbf{q}}(V) \otimes \mathbb{C} W \otimes S_{\mathbf{q}}\left(V^{*}\right)$ and fulfils the relations in Definition 3.2. To prove that $\underline{A}$ is a braided Cherednik algebra, we show that $A$ is a non-degenerate quadratic double if and only if the $\mathbf{q}$-commutator $[\cdot, \cdot]_{\mathbf{q}}$ between $V^{*}$ and $V$ in $\underline{A}$ has zero kernels in $V^{*}, V$.

Indeed, we have $\left[\underline{y}_{j}, x_{i}\right]_{\mathbf{q}}=\gamma_{j}^{-1} \cdot\left[y_{j}, x_{i}\right]$ for $x \in V$. Hence it is enough to show that the kernels of $[\cdot, \cdot]$ and $[\cdot, \cdot]_{\mathbf{q}}$ are spanned by $\Gamma_{\mathbf{q}}$-homogeneous elements. But observe that $\Gamma_{\mathbf{q}}$-homogeneous elements in $V^{*}$ and in $V$ are precisely the simultaneous eigenvectors for the action of $\Gamma_{\mathbf{q}}$. Furthermore, the kernels of $[\cdot, \cdot]$ and of $[\cdot, \cdot]_{\mathbf{q}}$ in $V^{*}$ and $V$ are $W$-submodules, therefore $\Gamma_{\mathbf{q}}$-submodules and thus spanned by eigenvectors for the action of $\Gamma_{\mathbf{q}}$, as required.

Second, assume that there is a braided Cherednik algebra of the form $S_{\mathbf{q}}(V) \otimes \mathbb{C} W \otimes S_{\mathbf{q}}\left(V^{*}\right)$. Then the group $W$ preserves the $\mathbf{q}$-exterior squares $\wedge_{\mathbf{q}}^{2} V$ and $\wedge_{\mathbf{q}}^{2} V^{*}$. Hence $W$ preserves $\wedge_{\mathbf{q}^{\top}}^{2} V^{*}=$ $\tau\left(\wedge_{\mathbf{q}}^{2} V^{*}\right)$ and, by Proposition 2.2, $W$ normalises $\Gamma_{\mathbf{q}}$. It follows that $\widetilde{W}:=W \cdot \Gamma_{\mathbf{q}}$ is a group which preserves $\wedge_{\mathbf{q}}^{2} V$ and $\wedge_{\mathbf{q}}^{2} V^{*}$. By Corollary 2.5, $V$ is a Yetter-Drinfeld module with respect to the action of $\widetilde{W}$ and the grading by elements of $\Gamma_{\mathbf{q}} \leq \widetilde{W}$. Put $y_{i}=\gamma_{i} \underline{y}_{i}$; then $x_{1}, \ldots, x_{n}, y_{1}, \ldots, y_{n}$ and $\widetilde{W}$ generate a quadratic double $A$, as shown in Proposition 1.22, of the form $S_{\mathbf{q}}(V) \otimes \mathbb{C} \widetilde{W} \otimes S_{\mathbf{q}^{\top}}\left(V^{*}\right)$. Our braided Cherednik algebra is the braided reduction of $A$. Moreover, by what we have already proved, $A$ is a non-degenerate quadratric double, i.e. the commutator $[\cdot, \cdot]$ between $V^{*}$ and $V$ has zero kernels, because this is true for $[\cdot, \cdot]_{\mathbf{q}}$.

The Proposition and its proof imply a $W$-equivariance condition for the braided commutator:

Corollary 3.4. Let $\underline{\mathcal{H}}(W)=S_{\mathbf{q}}(V) \otimes \mathbb{C} W \otimes S_{\mathbf{q}}\left(V^{*}\right)$ be a braided Cherednik algebra of a group $W \subset \mathrm{GL}(V)$, associated to a matrix $\mathbf{q}$. Then the braided commutator $[\cdot, \cdot]_{\mathbf{q}}: V^{*} \otimes V \rightarrow \mathbb{C W}$ is $W$-equivariant and $\Gamma_{\mathbf{q}}$-equivariant. (The action of both $W$ and $\Gamma_{\mathbf{q}}$ on $V^{*} \otimes V$ is standard diagonal, and on $\mathbb{C} W$ is by conjugation inside $\mathrm{GL}(V)$.)

Proof. As in the proof of Proposition 3.3, put $\widetilde{W}=W \cdot \Gamma_{\mathbf{q}}$ and view $\underline{\mathcal{H}}(W)$ as the braided reduction of $\mathcal{H}(\widetilde{W})=S_{\mathbf{q}}(V) \otimes \mathbb{C} W \otimes S_{\mathbf{q}^{\top}}\left(V^{*}\right)$. To compute the braided commutator of $\underline{y} \in V^{*}$ and $x \in V$ in $\underline{\mathcal{H}}(W)$, we assume $\underline{y}$ to be $\Gamma_{\mathbf{q}}$-homogeneous, put $\underline{y}=|y| y$ and write $[\underline{y}, x]_{\mathbf{q}}=[|y| y, x]_{\mathbf{q}}=|y|[y, x]$ precisely as in Proposition 1.22. Now for any $w \in \widetilde{W}$ we have

$$
[w(\underline{y}), w(x)]_{\mathbf{q}}=|w(y)|[w(y), w(x)]=w|y| w^{-1} \cdot w[y, x] w^{-1}=w|y|[y, x] w^{-1}=w[\underline{y}, x]_{\mathbf{q}} w^{-1}
$$

because $V^{*}$ is a Yetter-Drinfeld module for $\widetilde{W}$ and the commutator $[\cdot, \cdot]$ in $\mathcal{H}(\widetilde{W})$ is $\widetilde{W}$-invariant. Extending to arbitrary $\underline{y}$ by linearity, we obtain $\widetilde{W}=W \cdot \Gamma_{\mathbf{q}}$-equivariance of $[\cdot, \cdot]_{\mathbf{q}}$. 
Note that the group $W$ may not be stable under conjugation by $\Gamma_{\mathbf{q}}$, but the braided commutator must still be $\Gamma_{\mathbf{q}}$-equivariant.

3.3. Negative braided Cherednik algebras. Clearly, if $q_{i j}=1$ for all $i, j$, braided Cherednik algebras associated to $\mathbf{q}$ are ordinary rational Cherednik algebras. We will now construct a family of braided Cherednik algebras of finite groups with

$$
q_{i j}=-1 \quad \text { for all } i, j=1, \ldots, n, i \neq j .
$$

The matrix with such entries was denoted $\mathbf{- 1}$ in the Introduction. Recall, also from the Introduction,

- the elements $\sigma_{i j}^{(\varepsilon)}$ of order 4 in $\operatorname{GL}(V)$, defined for indices $i \neq j$ and for $\varepsilon \in \mathbb{C}^{\times}$;

— the finite group $W_{\mathcal{C}, \mathcal{C}^{\prime}}=\left\langle\left\{\sigma_{i j}^{(\varepsilon)} \mid \varepsilon \in \mathcal{C}\right\} \cup\left\{t_{i}^{\left(\varepsilon^{\prime}\right)} \mid \varepsilon^{\prime} \in \mathcal{C}^{\prime}\right\}\right\rangle \leq \mathrm{GL}(V)$, where $\mathcal{C}^{\prime} \subset \mathcal{C}$ are finite subgroups of $\mathbb{C}^{\times}$such that $|\mathcal{C}|$ is even.

We will write $W_{\mathcal{C}, \mathcal{C}^{\prime}}(n)$ to emphasise that there is a separate group $W_{\mathcal{C}, \mathcal{C}^{\prime}}$ in each $\operatorname{rank} n=\operatorname{dim} V$. We note that $W_{\mathcal{C}, \mathcal{C}^{\prime}}$ is an irreducible linear group, i.e., it irreducibly acts on $V$, and keep in mind that $W_{\mathcal{C}, \mathcal{C}^{\prime}}(n)$ is one of the groups $G(m, p, n)$ or $G(m, p, n)_{+}$with such $m$ and $p$ as described in the Introduction.

Definition 3.5. Fix a scalar function $c: \mathcal{C}^{\prime} \rightarrow \mathbb{C}$. The negative braided Cherednik algebra $\underline{\mathcal{H}}_{c}\left(W_{\mathcal{C}, \mathcal{C}^{\prime}}\right)$ is the algebra generated by $V=\operatorname{span}\left(x_{1}, \ldots, x_{n}\right), W_{\mathcal{C}, \mathcal{C}^{\prime}}$ and $V^{*}=\operatorname{span}\left(\underline{y}_{1}, \ldots, \underline{y}_{n}\right)$ subject to relations

(i) $x_{i} x_{j}+x_{j} x_{i}=\underline{y}_{i} \underline{y}_{j}+\underline{y}_{j} \underline{y}_{i}=0$ for all $i \neq j$;

(ii) $w x_{i} w^{-1}=w\left(x_{i}\right), w \underline{y}_{i} w^{-1}=w\left(\underline{y}_{i}\right)$;

(iii) $\underline{y}_{j} x_{i}+x_{i} \underline{y}_{j}=c_{1} \sum_{\varepsilon \in \mathcal{C}} \varepsilon \sigma_{i j}^{(\varepsilon)}$ for all $i \neq j$,

$$
\underline{y}_{i} x_{i}-x_{i} \underline{y}_{i}=1+c_{1} \sum_{j \neq i, \varepsilon \in \mathcal{C}} \sigma_{i j}^{(\varepsilon)}+\sum_{\varepsilon^{\prime} \in \mathcal{C}^{\prime} \backslash\{1\}} c_{\varepsilon^{\prime}} t_{i}^{\left(\varepsilon^{\prime}\right)},
$$

where $i, j=1, \ldots, n$ and $w \in W_{\mathcal{C}, \mathcal{C}^{\prime}}$.

Proposition 3.6. $\underline{\mathcal{H}}_{c}\left(W_{\mathcal{C}, \mathcal{C}^{\prime}}\right)$ is a braided Cherednik algebra.

Proof. The matrix $\mathbf{q}$ is given by $\mathbf{q}=\mathbf{- 1}$. Note that all indices form a single negative block with respect to $\mathbf{- 1}$.

Identify the group $\mathrm{GL}(V)$ with $\mathrm{GL}_{n}(\mathbb{C})$ via the basis $\left\{x_{i}\right\}$ of $V$. Let $m=|\mathcal{C}|$. Take $\widetilde{W}$ to be the complex reflection group $G(m, 1, n)$ of matrices in $\mathrm{GL}_{n}(\mathbb{C})$ with precisely $n$ non-zero entries, all of which are $m$ th roots of unity. Note that

$$
s_{i j}^{(\varepsilon)}:=(i j) t_{i}^{(\varepsilon)} t_{j}^{\left(\varepsilon^{-1}\right)}
$$

and $t_{i}^{(\varepsilon)}, \varepsilon \in \mathcal{C}$, are complex reflections in $\widetilde{W}$. Let $\mathcal{H}(\widetilde{W})$ be the algebra generated by $V, \widetilde{W}$ and $V^{*}$ subject to the relations in Proposition 2.9 (with $q_{i j}=-1$ for all $i \neq j !$ ) and the commutation 
relation

$$
\begin{aligned}
& y_{j} x_{i}-x_{i} y_{j}=(-\mathrm{id}) \cdot c_{1} \sum_{\varepsilon \in \mathcal{C}}-\varepsilon s_{i j}^{(\varepsilon)} \text { if } i \neq j, \\
& y_{i} x_{i}-x_{i} y_{i}=(-\mathrm{id}) \cdot\left(t_{i}^{(-1)}+c_{-1} \cdot 1-c_{1} \sum_{j \neq i, \varepsilon \in \mathcal{C}} s_{i j}^{(\varepsilon)}+\sum_{\varepsilon^{\prime} \in \mathcal{C}^{\prime} \backslash\{ \pm 1\}} c_{\varepsilon^{\prime}} t_{i}^{\left(-\varepsilon^{\prime}\right)}\right),
\end{aligned}
$$

where $\left(-\right.$ id) is the negative identity matrix in $\mathrm{GL}_{n}(\mathbb{C})$ (it is an element of $\widetilde{W}$ since $m$ is even). The coefficient $c_{-1}$ is assumed to be zero if $-1 \notin \mathcal{C}^{\prime}$. To observe that $\mathcal{H}(\widetilde{W})$ is a -1-Cherednik algebra, rewrite the commutation relation as

$$
\begin{aligned}
y x-x y= & (-\mathrm{id})\left(c_{-1}\langle x, y\rangle+\frac{1}{2} \sum_{i}\left\langle x, 2 y_{i}\right\rangle\left\langle y, x_{i}\right\rangle t_{i}^{(-1)}\right. \\
& \left.+c_{1} \sum_{i \neq j}\left\langle x, y_{i}-\varepsilon^{-1} y_{j}\right\rangle\left\langle x_{i}-\varepsilon x_{j}, y\right\rangle s_{i j}^{(\varepsilon)}+\sum_{i, \varepsilon^{\prime} \in \mathcal{C}^{\prime} \backslash\{ \pm 1\}} \frac{c_{\varepsilon^{\prime}}}{1+\varepsilon^{\prime}}\left\langle x,\left(1+\varepsilon^{\prime}\right) y_{i}\right\rangle\left\langle x_{i}, y\right\rangle t_{i}^{\left(-\varepsilon^{\prime}\right)}\right) .
\end{aligned}
$$

This is the same as the commutator in Theorem 2.14 given that there is only one block $B=$ $\{1, \ldots, n\}$ of indices which is negative, one has $\gamma_{B}=(-\mathrm{id})$. This commutator is non-degenerate because of the coefficient in front of $t_{i}^{(-1)}$, and is $\widetilde{W}$-equivariant, since $t_{i}^{(\varepsilon)}$ and $t_{j}^{(\delta)}$ are not conjugate in $\operatorname{GL}(V)$ if $\varepsilon \neq \delta$, and $s_{i j}^{(\varepsilon)}$ is never conjugate to $t_{k}^{\left(\varepsilon^{\prime}\right)}$ in $G(m, p, n)$ (see [DO, Section 3]).

Hence $\mathcal{H}(\widetilde{W})$ is a $\mathbf{- 1}$-Cherednik algebra by Corollary 2.16. It remains to observe that $\underline{\mathcal{H}}_{c}\left(W_{\mathcal{C}, \mathcal{C}^{\prime}}\right)$ is a braided reduction of $\mathcal{H}(\widetilde{W})$. Indeed, let $(-\mathrm{id}) \beta_{i j}$ be the commutator of $y_{j}$ and $x_{i}$ in $\mathcal{H}(\widetilde{W})$, defined above. By Proposition $\left[1.22\right.$, the braided commutator $\left[\underline{y}_{j}, x_{i}\right]_{-1}$ in the braided reduction of $\mathcal{H}(\widetilde{W})$ is equal to $\left|\underline{y}_{j}\right|(-\mathrm{id}) \beta_{i j}=\gamma_{j}^{-1}(-\mathrm{id}) \beta_{i j}$. Note that $\gamma_{j}^{-1}=\gamma_{j}$ acts on $x_{i}, i \neq j$, by -1 and on $x_{j}$ by 1 . Thus $\gamma_{j}^{-1} \cdot(-\mathrm{id})=t_{j}^{(-1)}$. We are left to note that $t_{j}^{(-1)} s_{i j}^{(\varepsilon)}=\sigma_{i j}^{(-\varepsilon)}$ and $t_{i}^{(-1)} t_{i}^{\left(\varepsilon^{\prime}\right)}=t_{i}^{\left(-\varepsilon^{\prime}\right)}$, therefore $\gamma_{j}^{-1}(-\mathrm{id}) \beta_{i j}$ is precisely the braided commutator of $\underline{y}_{j}$ and $x_{i}$ in Definition 3.5 .

Using the notation from the proof of Proposition 3.6, we can make another "change of variables" in the -1 -Cherednik algebra $\mathcal{H}(\widetilde{W})$ in the case when - id $\in \widetilde{W}$. Namely, $V$ becomes a Yetter-Drinfeld module for $\widetilde{W}$ via the grading $|v|=-$ id for all $v \in V$. By Proposition 1.22, the elements $z_{i}=$ $(-\mathrm{id}) \cdot y_{i} \in \mathcal{H}(\widetilde{W})$, together with the $x_{i}$ and $w \in W$, generate an algebra $\bar{H}(\widetilde{W})$ with relations

(i) $x_{i} x_{j}+x_{j} x_{i}=z_{i} z_{j}+z_{j} z_{i}=0$ for all $i \neq j$;

(ii) $w x_{i} w^{-1}=w\left(x_{i}\right), w z_{i} w^{-1}=w\left(z_{i}\right)$ for all $w \in W_{\mathcal{C}, \mathcal{C}^{\prime}}, i=1, \ldots, n$;

(iii) $z_{j} x_{i}+x_{i} z_{j}=c_{1} \sum_{\varepsilon \in \mathcal{C}}-\varepsilon s_{i j}^{(\varepsilon)}$ for all $i \neq j$, and

$$
z_{i} x_{i}+x_{i} z_{i}=t_{i}^{(-1)}+c_{-1} \cdot 1-c_{1} \sum_{j \neq i, \varepsilon \in \mathcal{C}} s_{i j}^{(\varepsilon)}+\sum_{\varepsilon^{\prime} \in \mathcal{C}^{\prime} \backslash\{ \pm 1\}} c_{\varepsilon^{\prime}} t_{i}^{\left(-\varepsilon^{\prime}\right)} \text { for } i=1, \ldots, n,
$$

obtained directly from the relations (因) and (因) in the proof of Proposition 3.6. We thus obtain

Corollary 3.7. The algebra with the above presentation (i)-(iii) has triangular decomposition $S_{-1}(V) \otimes \mathbb{C} \widetilde{W} \otimes S_{-1}\left(V^{*}\right)$. 
Remark 3.8 (The degenerate version). We introduce the "degenerate" negative braided Cherednik algebra $\underline{\mathcal{H}}_{0, c}\left(W_{\mathcal{C}, \mathcal{C}^{\prime}}\right)$ by omitting 1 from the commutator $\underline{y}_{i} x_{i}-x_{i} \underline{y}_{i}$ in Definition 3.5

$$
\underline{y}_{i} x_{i}-x_{i} \underline{y}_{i}=c_{1} \sum_{j \neq i, \varepsilon \in \mathcal{C}} \sigma_{i j}^{(\varepsilon)}+\sum_{\varepsilon^{\prime} \in \mathcal{C}^{\prime} \backslash\{1\}} c_{\varepsilon^{\prime}} t_{i}^{\left(\varepsilon^{\prime}\right)} .
$$

This is a braided Cherednik algebra, provided that the function $c$ is not identically zero. The proof is the same as for $\underline{\mathcal{H}}_{c}\left(W_{\mathcal{C}, \mathcal{C}^{\prime}}\right)$.

Remark 3.9 (The rank 2 case). It turns out that when $\operatorname{dim} V=2$, the definition of $\underline{\mathcal{H}}_{c}\left(W_{\mathcal{C}, \mathcal{C}^{\prime}}(2)\right)$ and $\underline{\mathcal{H}}_{c}\left(W_{\mathcal{C}, \mathcal{C}^{\prime}}(2)\right)$ can be modified to allow one extra degree of freedom in choosing the parameter $c$. We hereby modify Definition 3.5 to say that if $\operatorname{dim} V=2$, the algebra depends on $\left|\mathcal{C}^{\prime}\right|+1$ parameters $c_{1}, c_{1}^{\prime}, c_{\varepsilon^{\prime}}\left(\varepsilon^{\prime} \in \mathcal{C}^{\prime} \backslash\{1\}\right)$, and the commutation relations in $\underline{\mathcal{H}}_{c}\left(W_{\mathcal{C}, \mathcal{C}^{\prime}}(2)\right)$ will be

(iii) $\underline{y}_{j} x_{i}+x_{i} \underline{y}_{j}=c_{1} \sum_{\varepsilon \in \mathcal{C}^{2}} \varepsilon \sigma_{i j}^{(\varepsilon)}+c_{1} \sum_{\varepsilon \in \mathcal{C} \backslash \mathcal{C}^{2}} \varepsilon \sigma_{i j}^{(\varepsilon)}$ when $\{i, j\}=\{1,2\}$,

$$
\underline{y}_{i} x_{i}-x_{i} \underline{y}_{i}=1+c_{1} \sum_{j \neq i, \varepsilon \in \mathcal{C}^{2}} \sigma_{i j}^{(\varepsilon)}+c_{1}^{\prime} \sum_{j \neq i, \varepsilon \in \mathcal{C} \backslash \mathcal{C}^{2}} \sigma_{i j}^{(\varepsilon)}+\sum_{\varepsilon^{\prime} \in \mathcal{C}^{\prime} \backslash\{1\}} c_{\varepsilon^{\prime}} t_{i}^{\left(\varepsilon^{\prime}\right)} .
$$

Here $\mathcal{C}^{2}$ denotes the set of squares of elements of $\mathcal{C}$ (the only subgroup of index 2 in $\mathcal{C}$ ). The proof that $\underline{\mathcal{H}}_{c}\left(W_{\mathcal{C}, \mathcal{C}^{\prime}}(2)\right)$ is a braided Cherednik algebra is the same as in Proposition 3.6, but taking into account that in the complex reflection group $G(m, 1,2)$ the complex reflections $s_{12}^{(\varepsilon)}$ and $s_{12}^{\left(\varepsilon^{\prime}\right)}$ are conjugate if and only if $\varepsilon^{\prime}=\varepsilon \delta^{2}$ for some $\delta \in \mathcal{C}^{2}$.

It turns out that the algebras $\underline{\mathcal{H}}_{c}\left(W_{\mathcal{C}, \mathcal{C}^{\prime}}\right)$ and $\underline{\mathcal{H}}_{0, c}\left(W_{\mathcal{C}, \mathcal{C}^{\prime}}\right)$ exhaust all possible "negative braided" Cherednik algebra structures over the group $W_{\mathcal{C}, \mathcal{C}^{\prime}}$ :

Proposition 3.10. Any braided Cherednik algebra $\underline{\mathcal{H}}$ of the form $S_{-\mathbf{1}}(V) \otimes \mathbb{C} W_{\mathcal{C}, \mathcal{C}^{\prime}} \otimes S_{-\mathbf{1}}\left(V^{*}\right)$ is isomorphic to $\underline{\mathcal{H}}_{c}\left(W_{\mathcal{C}, \mathcal{C}^{\prime}}\right)$ or to $\underline{\mathcal{H}}_{0, c}\left(W_{\mathcal{C}, \mathcal{C}^{\prime}}\right)$ for some choice of the parameter $c$.

Proof. Let $\widetilde{W}=W_{\mathcal{C}, \mathcal{C}^{\prime}} \Gamma_{-\mathbf{1}}$, and consider a -1-Cherednik algebra $\mathcal{H}(\widetilde{W})$ such that $\underline{\mathcal{H}}$ is its braided reduction (as in the proof of Proposition $\underline{3.3}$ ). The braided commutator $\left[\underline{y}_{j}, x_{i}\right]_{-\mathbf{1}}$ in $\underline{\mathcal{H}}$ rewrites as $\gamma_{j}^{-1}\left[y_{j}, x_{i}\right]$, where $\gamma_{j}^{-1}=\gamma_{j}=t_{j}^{(-1)}$ and $\left[y_{j}, x_{i}\right]$ is the commutator in $\mathcal{H}(\widetilde{W})$, necessarily given by $\left[y_{j}, x_{i}\right]=(-\mathrm{id})\left(\right.$ scalar $\left.+\sum_{s} c_{s}\left\langle x_{i}, \alpha_{s}^{\vee}\right\rangle\left\langle\alpha_{s}, y_{i}\right\rangle s\right)$. Here $s$ runs over some complex reflections in the group $\widetilde{W}$, and $c_{s}$ are some scalars. Now observe that $\widetilde{W}$ is contained in the complex reflection group $G(m, 1, n)$ where $m=|\mathcal{C}|$. We know what are the complex reflections in $G(m, 1, n)$; it follows that for $i \neq j$, the only possible complex reflections appearing in the commutator $\left[y_{j}, x_{i}\right]$ are of the form $s_{i j}^{(\varepsilon)}$, and if $i=j$, then they can be of the form $t_{i}^{(\eta)}$ or $s_{i k}^{(\varepsilon)}$ for some $k \neq i$. We do not know what are the linear conditions on the scalars $c_{s}$, because this depends on how the complex reflections split into conjugacy classes in $\widetilde{W}$; but we certainly know that the coefficients of the same complex reflection $s_{i j}^{(\varepsilon)}$ in $\left[y_{j}, x_{i}\right]$ and in $\left[y_{i}, x_{i}\right]$ differ by the factor of $\varepsilon$.

All this is sufficient to determine that the cross-commutation relations in $\underline{\mathcal{H}}$ must be of the form 


$$
\begin{aligned}
& \underline{y}_{j} x_{i}+x_{i} \underline{y}_{j}=\left(\underline{y}_{j}, x_{i}\right)+\sum_{\varepsilon \in \mathcal{C}} \varepsilon a(i, j, \varepsilon) \sigma_{i j}^{(\varepsilon)}, \\
& \underline{y}_{i} x_{i}-x_{i} \underline{y}_{i}=\left(\underline{y}_{i}, x_{i}\right)+\sum_{j \neq i, \varepsilon \in \mathcal{C}} a(i, j, \varepsilon) \sigma_{i j}^{(\varepsilon)}+\sum_{\varepsilon^{\prime} \in \mathcal{C}^{\prime} \backslash\{1\}} b\left(i, \varepsilon^{\prime}\right) t_{i}^{\left(\varepsilon^{\prime}\right)}
\end{aligned}
$$

for some bilinear form $(\cdot, \cdot): V^{*} \otimes V \rightarrow \mathbb{C}$ and some coefficients $a(i, j, \varepsilon)(i \neq j), b(i, \varepsilon)$. Now we are going to use the $W_{\mathcal{C}, \mathcal{C}^{\prime}}$-equivariance of the braided commutator (Corollary 3.4). The form $(\cdot, \cdot)$ must be $W_{\mathcal{C}, \mathcal{C}^{\prime}}$-invariant, and as $W_{\mathcal{C}, \mathcal{C}^{\prime}}$ is an irreducible group, $(\cdot, \cdot)=\lambda\langle\cdot, \cdot\rangle$ is proportional to the evaluation pairing.

Equivariance of the second commutator formula with respect to $\sigma_{i 1}^{(1)}$ implies that $a(i, j, \varepsilon)=$ $a(1, j, \varepsilon)$ and $b\left(i, \varepsilon^{\prime}\right)=b\left(1, \varepsilon^{\prime}\right)$, and then equivariance under $\sigma_{2 j}^{(1)}$ implies that $a(1, j, \varepsilon)=a(1,2, \varepsilon)$. Finally, equivariance under $\sigma_{31}^{(1)} \sigma_{13}^{(\varepsilon)}=t_{1}^{\left(\varepsilon^{-1}\right)} t_{3}^{(\varepsilon)}$ implies the equation $a(1,2, \varepsilon)=a(1,2,1)$, because $t_{1}^{\left(\varepsilon^{-1}\right)} t_{3}^{(\varepsilon)} \sigma_{12}^{(1)}\left[t_{1}^{\left(\varepsilon^{-1}\right)} t_{3}^{(\varepsilon)}\right]^{-1}=\sigma_{12}^{(\varepsilon)}$. The same result can be obtained by using equivariance under $t_{1}^{\left(\sqrt{\varepsilon^{-1}}\right)}$. Thus, $a(i, j, \varepsilon)(\varepsilon \in \mathcal{C})$ are all equal to some constant $c_{1}$, and $b\left(i, \varepsilon^{\prime}\right)=c_{\varepsilon^{\prime}}\left(\varepsilon^{\prime} \in \mathcal{C}^{\prime}\right)$. One concludes that $\underline{\mathcal{H}} \cong \underline{\mathcal{H}}_{0, c}\left(W_{\mathcal{C}, \mathcal{C}^{\prime}}\right)$ if $\lambda=0$, or $\underline{\mathcal{H}} \cong \underline{\mathcal{H}}_{c}\left(W_{\mathcal{C}, \mathcal{C}^{\prime}}\right)$ if $\lambda \neq 0$, where $c$ is the function $\varepsilon^{\prime} \mapsto c_{\varepsilon^{\prime}}$ on $\mathcal{C}^{\prime}$.

The above argument only fails if the group $W_{\mathcal{C}, \mathcal{C}^{\prime}}$ does not contain $t_{1}^{\left(\varepsilon^{-1}\right)} t_{3}^{(\varepsilon)}$ and $\mathcal{C}^{\prime}$ does not contain $\sqrt{\varepsilon}$, for $\varepsilon \in \mathcal{C}$. This happens precisely when $\operatorname{dim} V=2$ (the rank 2 case). In this case, one may use equivariance of the braided commutator under $\sigma_{12}^{(\delta)}, \delta \in \mathcal{C}$, to establish $a(1,2, \varepsilon)=$ $a\left(1,2, \varepsilon^{-1} \delta^{2}\right)$ by observing that $\left(\sigma_{12}^{(\delta)}\right)^{-1} \sigma_{12}^{(\varepsilon)} \sigma_{12}^{(\delta)}=\sigma_{12}^{\left(\varepsilon^{-1} \delta\right)}$. In this case, the algebra will depend not on $\left|\mathcal{C}^{\prime}\right|$ but on $\left|\mathcal{C}^{\prime}\right|+1$ scalar parameters, as described in Remark 3.9.

Example 3.11 (Braided Cherednik algebra of type $B_{n}^{+}$). The smallest possible example of a nontrivial group $W_{\mathcal{C}, \mathcal{C}^{\prime}}$ in rank $n$ corresponds to $|\mathcal{C}|=2$ and $\left|\mathcal{C}^{\prime}\right|=1$. The group $G(2,1, n)$ is the Coxeter group of type $B_{n}$, and $W_{\{ \pm 1\},\{1\}}$ is the group of even elements in $B_{n}$. Denote this group by $B_{n}^{+}$. It is generated by $\sigma_{i j}, i, j=1, \ldots, n, i \neq j$, so that $\sigma_{i j}^{(1)}=\sigma_{i j}$ and $\sigma_{i j}^{(-1)}=\left(\sigma_{i j}\right)^{-1}=\sigma_{j i}$.

The following is the list of relations in the negative braided Cherednik algebra of type $B_{n}^{+}$:

- $x_{i} x_{j}+x_{j} x_{i}=\underline{y}_{i} \underline{y}_{j}+\underline{y}_{j} \underline{y}_{i}=0$ for $i \neq j$;

- $\sigma_{i j} x_{i}=x_{j} \sigma_{i j}, \quad \sigma_{i j} x_{j}=-x_{i} \sigma_{i j}, \quad \sigma_{i j} x_{k}=x_{k} \sigma_{i j}$ for $k \neq i, j$, and same with $\underline{y}_{i}$ in lieu of $x_{i}$;

- $\underline{y}_{j} x_{i}+x_{i} \underline{y}_{j}=c\left(\sigma_{i j}-\sigma_{j i}\right)$ for $i \neq j$;

- $\underline{y}_{i} x_{i}-x_{i} \underline{y}_{i}=1+c \sum_{j \neq i}\left(\sigma_{i j}+\sigma_{j i}\right)$.

\section{Classification of Braided Cherednik algebras}

In this Section, we classify braided Cherednik algebras of finite groups (under a natural minimality assumption on the group $W$ ). We do this by showing that they are braided tensor products of rational Cherednik algebras of irreducible complex reflection groups and negative braided Cherednik algebras of groups $G(m, p, n)$ and $G(m, p, n)_{+}$, introduced in the previous section. 
4.1. Braided tensor product of algebras. For $k=1, \ldots, m$, let $\mathcal{H}_{k}$ be a braided Cherednik algebra of a finite group $W_{k} \subset \mathrm{GL}\left(V_{k}\right)$, associated to a matrix $\mathbf{q}_{k}$ of size $n_{k} \times n_{k}$ where $n_{k}=\operatorname{dim} V_{k}$. We would like to turn the vector space $\underline{\mathcal{H}}_{1} \otimes \ldots \otimes \underline{\mathcal{H}}_{m}$ into a braided Cherednik algebra associated to a matrix $\mathbf{q}$ of size $n=\sum_{k} n_{k}$, with submatrices $\mathbf{q}_{k}$ along the main diagonal. However, the standard tensor product $A \otimes B$ of algebras where $a \in A$ and $b \in B$ commute, is not general enough because it would only give matrix $\mathbf{q}$ with all entries outside the submatrices $\mathbf{q}_{k}$ equal to 1 .

It turns out that the appropriate tensor multiplication here is the braided tensor product of algebras, well known in the theory of braided monoidal categories; see [M2]. Let us recall this notion without going into too much detail. Let $\mathbf{C}$ be a braided tensor category, i.e., for each pair $X$, $Y$ of objects there is a braiding $\Psi_{X, Y}: X \otimes Y \rightarrow Y \otimes X$ which is a morphism in C; these morphisms satisfy axioms of the categorical braiding. An algebra in $\mathbf{C}$ is an object $A$ of $\mathbf{C}$ equipped with associative multiplication $m_{A}: A \otimes A \rightarrow A$ and the unit map $1_{A}: \mathbb{I} \rightarrow A$ that are morphisms in $\mathbf{C}$, where $\mathbb{I}$ is the unit object in the category. The braided tensor product of algebras $A$ and $B$ in $\mathbf{C}$ is

$$
\begin{gathered}
A \underline{\otimes} B:=A \otimes B \text { as an object of } \mathbf{C} ; \\
m_{A \underline{\otimes} B}=\left(m_{A} \otimes m_{B}\right)\left(\operatorname{id}_{A} \otimes \Psi_{B, A} \otimes \operatorname{id}_{B}\right): A \otimes B \otimes A \otimes B \rightarrow A \otimes B, \\
1_{A} \underline{\otimes} B=1_{A} \otimes 1_{B} .
\end{gathered}
$$

The categorical braiding axioms ensure that $m_{A \otimes B}$ is an associative multiplication.

4.2. The braided tensor category $\mathcal{M}_{\Gamma, \mathcal{R}}$. The category ${ }_{\Gamma}^{\Gamma} \mathcal{Y D}$ of Yetter-Drinfeld modules (as introduced in Section (1) over a group $\Gamma$ is a braided category, with braiding

$$
X, Y \in \mathrm{Ob} \underset{\Gamma}{\Gamma} \mathcal{Y \mathcal { D }} \quad \mapsto \quad \Psi_{X, Y}: X \otimes Y \rightarrow Y \otimes X, \quad \Psi_{X, Y}(x \otimes y)=|x|(y) \otimes x .
$$

Our main example of a braided category will, however, be slightly different. Let $\Gamma$ be an Abelian group. Fix a map $\mathcal{R}: \Gamma \times \Gamma \rightarrow \mathbb{C}^{\times}$which is a unitary bicharacter, i.e., $\mathcal{R}(\gamma, \cdot)$ is a homomorphism from $\Gamma$ to $\mathbb{C}^{\times}$for fixed $\gamma \in \Gamma$, and $\mathcal{R}\left(\gamma, \gamma^{\prime}\right)=\mathcal{R}\left(\gamma^{\prime}, \gamma\right)^{-1}$. Assume that $\mathcal{R}$ is non-degenerate, that is, $\gamma \mapsto \mathcal{R}(\gamma, \cdot)$ is an embedding of $\Gamma$ in the group $\widehat{\Gamma}$ of its multiplicative characters. Denote by $\widehat{\Gamma}_{\mathcal{R}}$ the image of this embedding. Elements of $\widehat{\Gamma}_{\mathcal{R}}$ are viewed as 1-dimensional $\Gamma$-modules.

Definition 4.1 (The category $\mathcal{M}_{\Gamma, \mathcal{R}}$ ). Define the category $\mathcal{M}_{\Gamma, \mathcal{R}}$ as a full subcategory of $\Gamma$-modules consisting of objects isomorphic to direct sums of modules in $\widehat{\Gamma}_{\mathcal{R}}$. Each module $X$ in $\mathcal{M}_{\Gamma, \mathcal{R}}$ is $\Gamma$-graded by

$$
x \in X, \quad g(x)=\mathcal{R}(\gamma, \cdot) x \quad \forall g \in \Gamma \quad \Rightarrow \quad|x|=\gamma .
$$

It is clear that such grading makes $X$ a Yetter-Drinfeld module so that $\mathcal{M}_{\Gamma, \mathcal{R}}$ is a full subcategory of ${ }_{\Gamma}^{\Gamma} \mathcal{Y D}$ and defines the braiding $\Psi_{X, Y}$ between $X$ and $Y$ in $\mathcal{M}_{\Gamma, \mathcal{R}}$. 
In what follows, $\Gamma$ will be an Abelian group with fixed unitary non-degenerate bicharacter $\mathcal{R}$ on $\Gamma$. For $X \in \mathrm{Ob} M_{\Gamma, \mathcal{R}}$, denote

$$
|X|=\{\gamma \in \Gamma: \text { there exists } x \in X, x \neq 0,|x|=\gamma\} .
$$

4.3. Braided tensor product of braided Cherednik algebras. We will now observe that if $\Gamma$ acts on a braided Cherednik algebra $\underline{\mathcal{H}}(W)$ in a certain standard way, $\underline{\mathcal{H}}(W)$ is guaranteed to be a $\Gamma$-module algebra.

Definition 4.2. Let $\underline{\mathcal{H}}(W) \cong S_{\mathbf{q}}(V) \otimes \mathbb{C} W \otimes S_{\mathbf{q}}\left(V^{*}\right)$ be a braided Cherednik algebra, where $V$, as usual, is spanned by $x_{1}, \ldots, x_{n}$. A $\Gamma$-structure on $\underline{\mathcal{H}}(W)$ is a representation $\rho: \Gamma \rightarrow \operatorname{GL}(V)$ such that:

- $V$ becomes an object of $\mathcal{M}_{\Gamma, \mathcal{R}}$, and $x_{i} \in V$ are simultaneous eigenvectors for $\rho(\Gamma)$;

- $g^{-1} w^{-1} g w \in W \cap \rho(\Gamma)$ for all $g \in \rho(\Gamma), w \in W$. In particular, $(\gamma, w) \mapsto \rho(\gamma) w \rho(\gamma)^{-1}$ is a $\Gamma$-action on $W$;

— the braided commutator $[\cdot, \cdot]_{\mathbf{q}}: V^{*} \otimes V \rightarrow \mathbb{C} W$ is equivariant with respect to the $\Gamma$-action.

Lemma 4.3. Let $\underline{\mathcal{H}}(W)$ be a braided Cherednik algebra with $\Gamma$-structure. Then:

(a) $W$ acts on the set $|V| \subset \Gamma$;

(b) $\mathbb{C} W$ is a $\Gamma$-submodule of $\underline{\mathcal{H}}(W)$, and $|\mathbb{C} W|=\left\{\gamma^{-1} \cdot w(\gamma): \gamma \in|V|\right\}$;

(c) $\underline{\mathcal{H}}(W)$ is an algebra in the category $\mathcal{M}_{\Gamma, \mathcal{R}}$.

Proof. (a) If $x \in V$ is a simultaneous eigenvector for $\rho(\Gamma)$, and $w$ is in $W$, then $w(x)$ is a simultaneous eigenvector for $w \rho(\Gamma) w^{-1}$ corresponding to the same eigencharacter. But $w \rho(\Gamma) w^{-1}=\rho(\Gamma)$ by definition of $\Gamma$-structure. Thus, the action of $W$ permutes $\rho(\Gamma)$-simultaneous eigenspaces in $V$, which are $\Gamma$-homogeneous components of $V$, hence $W$ permutes $\Gamma$-degrees of elements of $V$. Note that the action of $W$ on the set $|V|$ is such that $w\left(\left|x_{i}\right|\right)=\left|w\left(x_{i}\right)\right|$.

(b) Consider the $\Gamma$-action on $\operatorname{End}(V)$ given by $(\gamma, m) \mapsto \rho(\gamma) m \rho(\gamma)^{-1}$ for $m \in \operatorname{End}(V)$. It is then easy to see that the canonical isomorphism $\operatorname{End}(V) \cong V \otimes V^{*}$ is an isomorphism of $\Gamma$-modules. Let $\left\{\underline{y}_{i}\right\}$, as usual, be the basis of $V^{*}$ dual to $\left\{x_{i}\right\}$. Then $x_{i} \otimes \underline{y}_{j} \in V \otimes V^{*}$ is a simultaneous eigenvector for $\Gamma$ of $\Gamma$-degree $\left|x_{i}\right|\left|x_{j}\right|^{-1}$. An element $w \in W$ is written as $\sum_{i=1}^{n} w\left(x_{i}\right) \otimes \underline{y}_{i} \in V \otimes V^{*}$, and the $\Gamma$-degree of $w\left(x_{i}\right) \otimes \underline{y}_{i}$ is $w\left(\left|x_{i}\right|\right) \cdot\left|x_{i}\right|^{-1}$. Thus, $\Gamma$-degrees that appear in the $\Gamma$-submodule of $\mathbb{C} W$ generated by $w$ are of the form $w\left(\left|x_{i}\right|\right) \cdot\left|x_{i}\right|^{-1}$, and the linear independence of $w\left(x_{i}\right) \otimes \underline{y}_{i}$ in the expansion of $w$ implies that all these $\Gamma$-degrees actually appear in this submodule.

(c) Thus, $\Gamma$ acts on generators of $\underline{\mathcal{H}}(W)$, and we check that this action preserves the relations in $\underline{\mathcal{H}}(W)$. The relation $w x w^{-1}=w(x)$ where $x \in V$ and $w \in W$ becomes $\rho(\gamma) w \rho(\gamma)^{-1} \cdot \rho(\gamma)(x)$. $\rho(\gamma) w^{-1} \rho(\gamma)^{-1}=(\rho(\gamma) w)(x)$, i.e., $w^{\prime} x^{\prime} w^{-1}=w^{\prime}\left(x^{\prime}\right)$ where $w^{\prime}=\rho(\gamma) w \rho(\gamma)^{-1} \in W$ and $x^{\prime}=$ $\rho(\gamma)(x)$. This is also a relation in $\underline{\mathcal{H}}(W)$. The relations $x_{i} x_{j}=q_{i j} x_{j} x_{i}$ are preserved since the 
$x_{i}$ are simultaneous eigenvectors of $\Gamma$. Similarly, the relations $w \underline{y} w^{-1}=w(\underline{y})$ for $\underline{y} \in V^{*}$, and $\underline{y}_{i} \underline{y}_{j}=q_{i j} \underline{y}_{j} \underline{y}_{i}$, are preserved. Finally, the braided commutation relations between $\underline{y}_{j}$ and $x_{i}$ are preserved because the braided commutator between $V^{*}$ and $V$ is $\Gamma$-equivariant.

The $\Gamma$-structure paves the way for introducing braided tensor product $\underline{\otimes}$ of braided Cherednik algebras. (Obviously, the usual tensor product where the two tensorands commute is a particular case of this, corresponding to the trivial " $\{1\}$-structure" on any braided Cherednik algebra.) Let us write down the triangular decomposition property of the braided tensor product:

Lemma 4.4. Let $\underline{\mathcal{H}}(W) \cong S_{\mathbf{q}}(V) \otimes \mathbb{C} W \otimes S_{\mathbf{q}}\left(V^{*}\right)$ and $\underline{\mathcal{H}}\left(W^{\prime}\right) \cong S_{\mathbf{q}^{\prime}}\left(V^{\prime}\right) \otimes \mathbb{C} W^{\prime} \otimes S_{\mathbf{q}^{\prime}}\left(V^{\prime *}\right)$ be braided Cherednik algebras with $\Gamma$-structure, where $V$ is spanned by variables $x_{1}, \ldots, x_{n}$ and $V^{\prime}$ is spanned by variables $x_{n+1}, \ldots, x_{n+m}$. Let $\mathcal{R}$ be a non-degenerate unitary bicharacter on $\Gamma$. The braided tensor product of $\underline{\mathcal{H}}(W)$ and $\underline{\mathcal{H}}\left(W^{\prime}\right)$ in the category $\mathcal{M}_{\Gamma, \mathcal{R}}$ has triangular decomposition

$$
\underline{\mathcal{H}}(W) \underline{\otimes} \underline{\mathcal{H}}\left(W^{\prime}\right) \cong S_{\widetilde{\mathbf{q}}}\left(V \oplus V^{\prime}\right) \otimes\left(\mathbb{C} W \underline{\otimes} \mathbb{C} W^{\prime}\right) \otimes S_{\widetilde{\mathbf{q}}}\left(V^{*} \oplus V^{\prime *}\right) .
$$

The $(n+m) \times(n+m)$ matrix $\widetilde{\mathbf{q}}=\left(\widetilde{q}_{i j}\right)$ is given by

$$
\widetilde{q}_{i j}=q_{i j}(i, j \leq n), \quad \widetilde{q}_{i j}=q_{i j}^{\prime}(n<i, j), \quad \widetilde{q}_{i j}=\mathcal{R}\left(g_{i}, g_{j}\right) \text { (otherwise), }
$$

where $g_{i}=\left|x_{i}\right|$ is the $\Gamma$-degree of $x_{i}$; in particular, $\widetilde{q}_{i j}=\widetilde{q}_{i j}^{-1}$.

Remark 4.5. Warning: $\mathbb{C} W \underline{\mathbb{C}} W^{\prime}$ is not necessarily a group algebra!

Proof of the Lemma. It is clear that we may write $\underline{\mathcal{H}}(W) \underline{\otimes} \underline{\mathcal{H}}\left(W^{\prime}\right)$ as a tensor product

$$
\left(S_{\mathbf{q}}(V) \otimes S_{\mathbf{q}^{\prime}}(V)\right) \otimes\left(\mathbb{C} W \otimes \mathbb{C} W^{\prime}\right) \otimes\left(S_{\mathbf{q}}\left(V^{*}\right) \otimes S_{\mathbf{q}^{\prime}}\left(V^{\prime *}\right)\right)=: U^{-} \otimes U^{0} \otimes U^{+}
$$

of subalgebras. The subalgebra $U^{-}$is generated by $x_{1}, \ldots, x_{n+m}$ modulo the relations

$$
x_{i} x_{j}=q_{i j} x_{j} x_{i}(i, j \leq n), \quad x_{i} x_{j}=q_{i j}^{\prime} x_{j} x_{i}(n<i, j), \quad x_{i} x_{j}=\mathcal{R}\left(g_{i}, g_{j}\right) x_{j} x_{i} \text { (otherwise), }
$$

the latter relation being dictated by the braided tensor product. Immediately $U^{-}=S_{\widetilde{\mathbf{q}}}\left(V \oplus V^{\prime}\right)$ as required. In the same way $U^{+}=S_{\widetilde{\mathbf{q}}}\left(V^{*} \oplus V^{\prime *}\right)$. Moreover, $U^{-} U^{0}$ is a subalgebra. This follows from the commutation relations $w x_{i}=w\left(x_{i}\right) w, w^{\prime} x_{k}=w^{\prime}\left(x_{k}\right) w$ where $i \leq n<k, w \in W$, $w^{\prime} \in W^{\prime}$, and some way (provided by the braided tensor product) to re-express the product $w^{\prime} x_{i}$ as an element in $V \otimes \mathbb{C} W^{\prime}$; and a way to re-express $w x_{k}$ as an element in $V^{\prime} \otimes \mathbb{C} W$. Similarly, $U^{0} U^{+}$ is a subalgebra.

In general, however, $\underline{\otimes}$ applied to braided Cherednik algebras $\underline{\mathcal{H}}(W)$ and $\underline{\mathcal{H}}\left(W^{\prime}\right)$ will not produce a braided Cherednik algebra, at least because the associative algebra $\mathbb{C} W \underline{\otimes} \mathbb{C} W^{\prime}$ may not be the group algebra of $W \times W^{\prime}$. This generalisation of braided (and in particular, rational) Cherednik algebras may deserve to be studied elsewhere. For the purposes of the present paper, we would 
like to force $\mathcal{H}(W) \underline{\otimes} \underline{\mathcal{H}}\left(W^{\prime}\right)$ to be a braided Cherednik algebra by some extra condition on the bicharacter $\mathcal{R}$ on $\Gamma$. Here is the criterion for the braided product of two braided Cherednik algebras to be a braided Cherednik algebra of the direct product of groups:

Proposition 4.6. In the notation of Lemma 4.3 and Lemma 4.4. $\underline{\mathcal{H}}(W) \underline{\otimes} \underline{\mathcal{H}}\left(W^{\prime}\right)$ is a braided Cherednik algebra of the group $W \times W^{\prime}$ acting on the space $V \oplus V^{\prime}$, if and only if $\mathcal{R}\left(w(\gamma), w^{\prime}\left(\gamma^{\prime}\right)\right)=$ $\mathcal{R}\left(\gamma, \gamma^{\prime}\right)$ for all $w \in W, w^{\prime} \in W^{\prime}, \gamma \in|V|, \gamma^{\prime} \in\left|V^{\prime}\right|$.

Proof. Clearly, $\underline{\mathcal{H}}(W) \underline{\otimes} \underline{\mathcal{H}}\left(W^{\prime}\right)$ is a braided Cherednik algebra of the group $W \times W^{\prime}$ acting on $V \oplus V^{\prime}$, only if the following relations hold in $\underline{\mathcal{H}}(W) \underline{\otimes} \underline{\mathcal{H}}\left(W^{\prime}\right)$ :

1. $w w^{\prime}=w^{\prime} w$ for $w \in W$ and $w^{\prime} \in W^{\prime}$, equivalent to $\mathbb{C} W \underline{\otimes} \mathbb{C} W^{\prime}$ being the group algebra of $W \times W^{\prime}$

2. $x_{k} w=w x_{k}(w \in W, n<k \leq n+m), w^{\prime} x_{i}=x_{i} w^{\prime}\left(w^{\prime} \in W^{\prime}, 1 \leq k \leq n\right)$, which are equivalent to the correct smash product relations between $W \times W^{\prime}$ and $x_{1}, \ldots, x_{n+m}$;

3. same as 2 ., but with $\underline{y}_{i}$ instead of $x_{i}$.

Let us observe that conditions 1.-3. are not only necessary but also sufficient. Indeed, the commutation relation in $\underline{\mathcal{H}}(W) \underline{\otimes} \underline{\mathcal{H}}\left(W^{\prime}\right)$ between $\underline{y}_{j}$ and $x_{i}$ where, say, $i \leq n<j$, is

$$
\underline{y}_{j} x_{i}=\mathcal{R}\left(g_{j}^{-1}, g_{i}\right) x_{i} \underline{y}_{j}=\widetilde{q}_{i j} x_{i} \underline{y}_{j} \quad \Leftrightarrow \quad\left[\underline{y}_{j}, x_{i}\right]_{\widetilde{\mathbf{q}}}=0
$$

where the matrix $\widetilde{\mathbf{q}}$ is given in Lemma 4.4 and the same holds for $j \leq n<i$. Thus, $\left[\underline{y}_{j}, x_{i}\right]_{\widetilde{\mathbf{q}}} \in$ $\mathbb{C} W \otimes \mathbb{C} W^{\prime}$ for all indices $i, j$. Besides that, the $\mathbb{C} W \otimes \mathbb{C} W^{\prime}$-valued braided commutator $[\cdot, \cdot \cdot]_{\widetilde{\mathbf{q}}}$ on $\left(V^{*} \oplus V^{\prime *}\right) \times\left(V \oplus V^{\prime}\right)$ has no left or right kernel, because it coincides with the non-degenerate commutator $[\cdot, \cdot]_{\mathbf{q}}$ (respectively $[\cdot, \cdot]_{\mathbf{q}^{\prime}}$ ) when restricted to $V^{*} \times V$ (respectively $\left.V^{\prime *} \times V^{\prime}\right)$ and has zero restriction to $V^{*} \times V^{\prime}$ and to $V^{*} \times V$. Thus, 1.-3. imply that $\underline{\mathcal{H}}(W) \underline{\otimes} \underline{\mathcal{H}}\left(W^{\prime}\right)$ is a braided Cherednik algebra associated to the matrix $\widetilde{\mathbf{q}}$.

But 1.-3. can clearly be rewritten as relations

$$
w^{\prime} a=a w^{\prime} \quad\left(a \in \underline{\mathcal{H}}(W), w^{\prime} \in W^{\prime}\right), \quad b w=w b \quad\left(b \in \underline{\mathcal{H}}\left(W^{\prime}\right), w \in W\right) .
$$

in the algebra $\underline{\mathcal{H}}(W) \underline{\otimes} \underline{\mathcal{H}}\left(W^{\prime}\right)$. In terms of the bicharacter $\mathcal{R}$ on $\Gamma$ these are equivalent to $\mathcal{R}(g, h)=1$ for all $g \in|\mathbb{C} W|, h \in\left|\underline{\mathcal{H}}\left(W^{\prime}\right)\right|$ and for all $g \in|\underline{\mathcal{H}}(W)|, h \in\left|\mathbb{C} W^{\prime}\right|$. This is precisely equivalent to the $\left(W, W^{\prime}\right)$-invariance of the restriction of $\mathcal{R}$ to $|V| \times\left|V^{\prime}\right|$, stated in the Proposition, because $|\mathbb{C} W|$ consists of $\gamma^{-1} w(\gamma)$ where $w$ runs over $W$ and $\gamma$ runs over $|V|$, and $\left|\underline{\mathcal{H}}\left(W^{\prime}\right)\right|$ lies in the subgroup of $\Gamma$ generated by $\left|V^{\prime}\right|$; similarly for $\left|\mathbb{C} W^{\prime}\right|$ and $|\underline{\mathcal{H}}(W)|$. 
4.4. Braided Cherednik algebras: the main structural theorem. Braided tensor multiplication is a powerful method of constructing new associative algebras. Even restricted by the orthogonality condition in Proposition 4.6, braided tensor multiplication is sufficient for obtaining essentially any braided Cherednik algebra of a finite group as a product of algebras from the following list:

(1) $H_{c}(W)$ where $W$ is an irreducible complex reflection group $G(m, p, n)$ or $G_{4}, \ldots, G_{37}$ in the Shephard-Todd classification [ST;

(2) $H_{0, c}(W), W$ same as in (1);

(3) $\underline{\mathcal{H}}_{c}(W)$, where $W$ is $G(m, p, n)$ with $m$ even, $\frac{m}{p}$ even, $n \geq 2$, or the subgroup $G(m, p, n)_{+}$ of even elements in $G(m, p, n)$ with $m$ even, $\frac{m}{2 p}$ odd, $n \geq 2$;

(4) $\underline{\mathcal{H}}_{0, c}(W), W$ same as in $(3)$.

We gave the definition of algebras $H_{c}(W)$ and $H_{0, c}(W)$ in Example1.13. These are rational Cherednik algebras, whereas $\underline{\mathcal{H}}_{c}(W)$ (Definition 3.5, Remark 3.9) and $\underline{\mathcal{H}}_{0, c}(W)$ (Remark 3.8) are negative braided Cherednik algebras. In other words, the (1)-(4) is the list of all rational and negative braided Cherednik algebras of irreducible groups.

"Essentially any braided Cherednik algebra" refers to $\underline{\mathcal{H}}(W)$ that satisfies the condition in

Definition 4.7. We say that the group $W$ is minimal for a braided Cherednik algebra $\underline{\mathcal{H}}(W) \cong$ $S_{\mathbf{q}}(V) \otimes \mathbb{C} W \otimes S_{\mathbf{q}}\left(V^{*}\right)$, if the image of the braided commutator $[\cdot, \cdot]_{\mathbf{q}}: V^{*} \times V \rightarrow \mathbb{C} W$ does not lie in $\mathbb{C} W_{1}$ for any proper subgroup $W_{1} \leq W$.

Every braided Cherednik algebra $\underline{\mathcal{H}}(W) \cong S_{\mathbf{q}}(V) \otimes \mathbb{C} W \otimes S_{\mathbf{q}}\left(V^{*}\right)$ contains a subalgebra given as $S_{\mathbf{q}}(V) \otimes \mathbb{C} W_{\min } \otimes S_{\mathbf{q}}\left(V^{*}\right)$ where $W_{\min }$ is the subgroup generated by elements of $W$ that appear in braided commutators between $V^{*}$ and $V$ with non-zero coefficients. Clearly, all relevant information about a braided Cherednik algebra $\underline{\mathcal{H}}(W)$ is contained in this subalgebra, the braided Cherednik algebra of $W_{\min }$. This is the reason why rational Cherednik algebras are often considered only over complex reflection groups. We apply the same principle to arbitrary braided Cherednik algebras:

Theorem 4.8. Any braided Cherednik algebra $\underline{\mathcal{H}}(W)$ where $W$ is minimal and finite, decomposes as a braided tensor product of algebras from (1)-(4) above in the category $\mathcal{M}_{\Gamma, \mathcal{R}}$ for some Abelian group $\Gamma$ and a non-degenerate unitary bicharacter $\mathcal{R}$.

Remark 4.9. The Theorem does not hold for q-Cherednik algebras.

Proof of Theorem 4.8. Fix the triangular decomposition $\underline{\mathcal{H}}(W) \cong S_{\mathbf{q}}(V) \otimes \mathbb{C} W \otimes S_{\mathbf{q}}\left(V^{*}\right)$ where $V$ is spanned by the variables $x_{1}, \ldots, x_{n}$ and $V^{*}$ is spanned by $\underline{y}_{1}, \ldots, \underline{y}_{n}$. We put $\Gamma=\Gamma_{\mathbf{q}}$; recall from Section 2 that $\Gamma_{\mathbf{q}}$ is the subgroup of GL $(V)$ generated by $\gamma_{1}, \ldots, \gamma_{n}$ where $\gamma_{i}\left(x_{j}\right)=q_{i j} x_{j}$. We let $\mathcal{R}=\mathcal{R}_{\mathbf{q}}$ be determined, via the extension to the whole of $\Gamma_{\mathbf{q}}$ by the bicharacter property, by 
$\mathcal{R}_{\mathbf{q}}\left(\gamma_{i}, \gamma_{j}\right)=q_{i j}$. It is easy to check that $\mathcal{R}_{\mathbf{q}}$ is a well-defined non-degenerate unitary bicharacter. In the course of the proof it will become apparent that the natural representation of $\Gamma_{\mathbf{q}}$ on $V$ is a $\Gamma_{\mathbf{q}}$-structure on $\underline{\mathcal{H}}(W)$.

Step 1: Factorisation into algebras $\mathcal{H}_{B}$ indexed by blocks $B$. Recall from Corollary 2.12 that the index set splits into disjoint blocks so that for $i \neq j$ one has $q_{i j}=q_{B, C}$ where $B, C$ are blocks, $i \in B, j \in C$. Each block $B$ is either positive $\left(q_{B, B}=1\right)$ or negative $(|B|>1$, $\left.q_{B, B}=-1\right)$. Recall from Proposition 3.3 that $\mathcal{H}(W)$ is a braided reduction of a q-Cherednik algebra $\mathcal{H}(\widetilde{W}) \cong S_{\mathbf{q}}(V) \otimes \mathbb{C} \widetilde{W} \otimes S_{\mathbf{q}^{\top}}\left(V^{*}\right)$. Here $\widetilde{W}$ is a group containing $W$ (we assume $\widetilde{W}=W \cdot \Gamma_{\mathbf{q}}$ as in the proof of Proposition 3.3 , and the basis of $V^{*}$ is now given by $y_{i}=\gamma_{i}^{-1} \underline{y}_{i}, i=1, \ldots, n$, so that

$$
C_{i j}:=\left[\underline{y}_{j}, x_{i}\right]_{\mathbf{q}}=\gamma_{j}^{-1}\left[y_{j}, x_{i}\right] \text {. }
$$

By Corollary 2.15 $y_{j}$ commutes with $x_{i}$ unless $i, j$ are in the same block. Equivalently,

$$
\underline{y}_{j} x_{i}=q_{i j} x_{i} \underline{y}_{j}=\mathcal{R}\left(\gamma_{j}^{-1}, \gamma_{i}\right) x_{i} \underline{y}_{j}
$$

if $i, j$ are not in the same block. If $i, j$ are in the same block (say $B$ ), Theorem 2.14 implies

$$
C_{i j}=\gamma_{j}^{-1} \gamma_{B}\left(a_{i j}+\sum_{s} b_{i, j, s} s\right)
$$

for certain constants $a_{i j}$ and $b_{i, j, s}$, where $s$ runs over a set of complex reflections in $\operatorname{GL}\left(V_{B}\right)$ with $V_{B}=$ $\oplus_{k \in B} \mathbb{C} x_{k}$. The group $\mathrm{GL}(V)$ contains the subgroup $\mathrm{GL}\left(V_{B_{1}}\right) \times \cdots \times \mathrm{GL}\left(V_{B_{N}}\right)$ where $B_{1}, \ldots, B_{N}$ are all blocks of indices, $B_{1} \cup \cdots \cup B_{N}=\{1, \ldots, n\}$. This corresponds to the direct sum decomposition $V=V_{B_{1}} \oplus \cdots \oplus V_{B_{N}}$. Note the crucial fact that

$$
\gamma_{j}^{-1} \gamma_{B}=1 \text { if the block } B \text { is positive, } \quad \gamma_{j}^{-1} \gamma_{B}=t_{j}^{(-1)} \text { if } B \text { is negative, }
$$

where $t_{j}^{(-1)}$ acts by -1 on $x_{j}$ and by 1 on the rest of the variables $x_{i}$. Thus, $t_{j}^{(-1)} \in \mathrm{GL}\left(V_{B}\right)$, and all elements of $W$ that appear in $C_{i j}(i, j \in B)$ with non-zero coefficients are elements of $\operatorname{GL}\left(V_{B}\right)$. By minimality, $W$ is generated by such elements, and hence has direct product decomposition

$$
W=W_{B_{1}} \times \cdots \times W_{B_{N}} \quad \subset \quad \operatorname{GL}\left(V_{B_{1}}\right) \times \cdots \times \operatorname{GL}\left(V_{B_{N}}\right), \quad W_{B_{k}}:=W \cap \operatorname{GL}\left(V_{B_{k}}\right) .
$$

It is now clear that $\underline{\mathcal{H}}(W)$ (as a vector space) has factorisation

$$
\underline{\mathcal{H}}(W)=\mathcal{H}_{B_{1}} \otimes \ldots \otimes \mathcal{H}_{B_{N}}
$$

where $\mathcal{H}_{B}$ is the subalgebra of $\underline{\mathcal{H}}(W)$ generated by $x_{i}, \underline{y}_{i}(i \in B)$ and $W_{B}$. If $B$ is a positive block, the algebra $\mathcal{H}_{B}$ has triangular decomposition $\mathcal{H}_{B} \cong S\left(V_{B}\right) \otimes \mathbb{C} W_{B} \otimes S\left(V_{B}^{*}\right)$ and is a rational Cherednik algebra of a complex reflection group $V_{B}$. If $B$ is negative block, $\mathcal{H}_{B}$ has triangular decomposition $S_{-\mathbf{1}}\left(V_{B}\right) \otimes \mathbb{C} W_{B} \otimes S_{-\mathbf{1}}\left(V_{B}^{*}\right)$. 
Let us show that the algebra $\underline{\mathcal{H}}(W)$ and all subalgebras $\mathcal{H}_{B}$ have $\Gamma_{\mathbf{q}}$-structure given by the action of $\Gamma_{\mathbf{q}}$ on $V$ (and hence on all $V_{B}$ that are $\Gamma_{\mathbf{q}}$-submodules of $V$ ). According to Definition 4.2 we have to check that

1. $\gamma w \gamma^{-1} w^{-1} \in W_{B} \cap \Gamma_{\mathbf{q}}$ for $\gamma \in \Gamma_{\mathbf{q}}, w \in W_{B}$;

2. the braided commutator $[\cdot, \cdot]_{\mathbf{q}}: V_{B}^{*} \times V_{B} \rightarrow \mathbb{C} W_{B}$ is $\Gamma_{\mathbf{q}}$-equivariant.

In 1., we already know that $W$ normalises $\Gamma_{\mathbf{q}}$ by Proposition 2.2, hence it is enough to check that $\Gamma_{\mathbf{q}}$ normalises $W_{B}$ in $\operatorname{GL}(V)$. But this follows from 2., because by minimality of $W, W_{B}$ is generated by elements of $\mathrm{GL}(V)$ that appear in the braided commutator $[\cdot, \cdot]_{\mathbf{q}}: V_{B}^{*} \times V_{B} \rightarrow \mathbb{C} W$. Of course, 2. is true by Corollary 3.4 .

Let us now show that $\mathcal{H}(W)$ is a tensor product of the $\mathcal{H}_{B}$ not only as a vector space but as an algebra in the category $\mathcal{M}_{\Gamma_{\mathbf{q}}}, \mathcal{R}_{\mathbf{q}}$. Since we already have the $\Gamma_{\mathbf{q}}$-structure on $\mathcal{H}_{B}$ and tensor factorisation of $\underline{\mathcal{H}}(W)$ into the algebras $\mathcal{H}_{B}$, it is enough to check that the commutation relations in $\mathcal{H}_{B} \otimes \mathcal{H}_{B^{\prime}}$ between $\mathcal{H}_{B}$ and $\mathcal{H}_{B^{\prime}}$ for blocks $B \neq B^{\prime}$ hold also in $\underline{\mathcal{H}}(W)$. By Lemma 4.4 the $x_{i}$ q-commute in $\mathcal{H}_{B} \underline{\otimes} \mathcal{H}_{B^{\prime}}$, as well as the $\underline{y}_{i}$; the same holds in $\underline{\mathcal{H}}(W)$. Furthermore, the definition of $\underline{\otimes}$ prescribes the relations $\underline{y}_{j} x_{i}=q_{i j} x_{i} \underline{y}_{j}$ in $\mathcal{H}_{B} \underline{\otimes} \mathcal{H}_{B^{\prime}}$ where $i \in B, j \in B^{\prime}$; we have already shown in this proof that the same holds in $\underline{\mathcal{H}}(W)$. Similarly for $i \in B^{\prime}, j \in B$. Finally, let us find the relations between $W_{B}$ and $\mathcal{H}_{B^{\prime}}$ and between $\mathcal{H}_{B}$ and $W_{B^{\prime}}$ in $\mathcal{H}_{B} \underline{\otimes} \mathcal{H}_{B^{\prime}}$. The group $W_{B}$ acts on $\left\{\gamma_{i} \mid i \in B\right\}$ by conjugation, and for two blocks $B \neq B^{\prime}$ we have

$$
\mathcal{R}_{\mathbf{q}}\left(w \gamma_{i} w^{-1}, w^{\prime} \gamma_{i^{\prime}} w^{\prime-1}\right)=\mathcal{R}_{\mathbf{q}}\left(\gamma_{i}, \gamma_{i^{\prime}}\right)=q_{i i^{\prime}}
$$

for all $w \in W_{B}, w^{\prime} \in W_{B^{\prime}}, i \in B, i^{\prime} \in B^{\prime}$ simply because $q_{j j^{\prime}}=q_{i i^{\prime}}$ for all $j \in B, j^{\prime} \in B^{\prime}$ by definition of a block. Therefore, by Proposition 4.6 $W_{B}$ commutes with $\mathcal{H}_{B^{\prime}}$ and $\mathcal{H}_{B}$ commutes with $W_{B^{\prime}}$ in $\mathcal{H}_{B} \underline{\otimes} \mathcal{H}_{B^{\prime}}$. But again, the same happens in $\underline{\mathcal{H}}(W)$. Hence the braided tensor product $\mathcal{H}_{B_{1}} \underline{\otimes} \ldots \underline{\otimes} \mathcal{H}_{B_{N}}$ indeed coincides with $\underline{\mathcal{H}}(W)$.

Step 2: Factorisation of $\mathcal{H}_{B}, B$ a positive block, into rational Cherednik algebras of irreducible groups. It remains to break up each of the algebras $\mathcal{H}_{B}$ into a braided tensor product, in the category $\mathcal{M}_{\Gamma_{\mathbf{q}}}, \mathcal{R}_{\mathbf{q}}$, of "elementary" braided Cherednik algebras from the list (1)(4). The more familiar case is that of a positive block, where $\mathcal{H}_{B}$ has triangular decomposition $S\left(V_{B}\right) \otimes \mathbb{C} W_{B} \otimes S\left(V_{B}^{*}\right)$ with commutation relation

$$
y x-x y=(x, y)_{B} \cdot 1+\sum_{s} c_{s}\left\langle x, \alpha_{s}^{\vee}\right\rangle\left\langle\alpha_{s}, y\right\rangle s,
$$

where $s$ runs over complex reflections in $W_{B}$, and $(\cdot, \cdot)_{B}$ is some $W_{B}$-invariant bilinear form on $V_{B} \times V_{B}^{*}$. By a known result on complex reflection groups, $W_{B} \subset \mathrm{GL}\left(V_{B}\right)$ is a direct product $W^{1} \times \cdots \times W^{l}$ of irreducible complex reflection groups corresponding to a direct sum decomposition 
$V_{B}=V^{1} \oplus \cdots \oplus V^{l}$. Denote by $\pi^{k}: V_{B} \rightarrow V^{k}$ the projection of $V$ onto its direct summand $V^{k}$. The dual space $V_{B}^{*}$ has the dual direct sum decomposition $V^{1 *} \oplus \cdots \oplus V^{l *}$ with $V^{k *}=\operatorname{im} \pi^{k *}$. Since $V^{k}$ are irreducible $W$-submodules of $V$, the $W$-invariant pairing $(\cdot, \cdot)_{B}$ between $V$ and $V^{*}$ is of the form $\sum_{k=1}^{l} \lambda_{k}\left\langle\pi^{k}(\cdot), \cdot\right\rangle$ for some $\lambda_{k} \in \mathbb{C}$. Moreover, any complex reflection $s \in W$ belongs to one of the $W^{k}$, thus $\alpha_{s} \in V^{k}$ and $\alpha_{s}^{\vee} \in V^{k *}$. It follows that $x \in V^{k}$ and $y \in V^{k^{\prime}}$ commute in $\mathcal{H}_{B}$ for $k \neq k^{\prime}$, and $\mathcal{H}_{B}$ decomposes as the tensor product

$$
\mathcal{H}_{B}=H\left(W^{1}\right) \otimes \ldots \otimes H\left(W^{l}\right)
$$

of commuting subalgebras. Here $H\left(W^{k}\right)=S\left(V^{k}\right) \otimes \mathbb{C} W^{k} \otimes S\left(V^{k *}\right)$ with the main commutation relation

$$
y x-x y=\lambda_{k}\langle x, y\rangle+\sum_{s \in W^{k}} c_{s}\left\langle x, \alpha_{s}^{\vee}\right\rangle\left\langle\alpha_{s}, y\right\rangle s
$$

between $y \in V^{k *}$ and $x \in V^{k}$, thus is a rational Cherednik algebra isomorphic to either $H_{0, c}\left(W^{k}\right)$ or $H_{c}\left(W^{k}\right)$, depending on whether $\lambda_{k}$ is zero or not.

It remains to note that the standard tensor product of commuting subalgebras is in this case the same as braided tensor product in the category $\mathcal{M}_{\Gamma_{\mathbf{q}}, \mathcal{R}_{\mathbf{q}}}$. First of all, $\Gamma_{\mathbf{q}}$ acts by scalars on $V_{B}$ and $V_{B}^{*}$, hence trivially on $W_{B}$. Thus $V^{k}, \mathbb{C} W^{k}$ and $V^{k *}$ are $\Gamma_{\mathbf{q}}$-submodules of $V_{B}, \mathbb{C} W_{B}$ and $V_{B}^{*}$, respectively. We now only need to check that the commutation relations between $H\left(W^{k}\right)$ and $H\left(W^{k^{\prime}}\right)$ inside $\mathcal{H}_{B}$ (where these two subalgebras commute) are the same as in the braided tensor product $H\left(W^{k}\right) \underline{\otimes} H\left(W^{k^{\prime}}\right)$. Note the $\Gamma_{\mathbf{q}}$-degrees that arise in the $\Gamma_{\mathbf{q}}$-module $\mathcal{H}_{B}$ lie in the subgroup of $\Gamma_{\mathbf{q}}$ generated by $\left\{\gamma_{i} \mid i \in B\right\}$; therefore, the value of $\mathcal{R}_{\mathbf{q}}$ on any two such degrees is 1 , because $\mathcal{R}_{\mathbf{q}}\left(\gamma_{i}, \gamma_{j}\right)=q_{i j}=1$ for any $i, j$ in the positive block $B$. Thus, $H\left(W^{k}\right)$ and $H\left(W^{k^{\prime}}\right)$ indeed commute in $H\left(W^{k}\right) \underline{\otimes} H\left(W^{k^{\prime}}\right)$.

Step 3: Factorisation of $\mathcal{H}_{B}, B$ a negative block, into braided Cherednik algebras $\underline{\mathcal{H}}\left(W^{k}\right)$. Now assume that $B$ is a negative block. The group $W_{B}$ may not be a complex reflection group. By an observation at Step 1 of this proof, Theorem 2.14 and Lemma 2.13(3), $W_{B}$ is generated by some elements of the form

$$
t_{j}^{(-1)}, \quad t_{j}^{(-1)} t_{j}^{(\eta)}(\eta \neq 1 \text { root of unity }), \quad \text { or } \quad t_{j}^{(-1)}(i j) t_{i}^{(\varepsilon)} t_{j}^{\left(\varepsilon^{-1}\right)}\left(\varepsilon \in \mathbb{C}^{\times}\right), \quad i, j \in B,
$$

besause only such elements may appear in $C_{i j}(i, j \in B)$ with nonzero coefficients. We rewrite the list of possible generators of $W_{B}$ as

$$
t_{j}^{(\eta)} \quad(\eta \text { any root of unity }), \quad \sigma_{i j}^{(\varepsilon)} \quad\left(\varepsilon \in \mathbb{C}^{\times}\right), \quad i, j \in B .
$$


Call two indices $i, j \in B$ linked, if $i=j$ or $W_{B}$ contains an element $\sigma_{i j}^{(\varepsilon)}$ for some $\varepsilon \in \mathbb{C}^{\times}$. The relation "linked" is symmetric and transitive, because

$$
\sigma_{i j}^{(\varepsilon)}=\sigma_{j i}^{\left(-\varepsilon^{-1}\right)}, \quad\left(\sigma_{i j}^{(\varepsilon)}\right)^{-1} \sigma_{j k}^{(\delta)} \sigma_{i j}^{(\varepsilon)}=\sigma_{i k}^{(\varepsilon \delta)} .
$$

Let $B=\mathcal{O}^{1} \cup \cdots \cup \mathcal{O}^{l}$ be the partition of $B$ into equivalence classes, and denote $V^{k}=\oplus\left\{\mathbb{C} x_{i} \mid i \in\right.$ $\left.\mathcal{O}^{k}\right\}$. The generating set for $W_{B}$ is partitioned into pairwise commuting subsets $\left\{t_{j}^{(\eta)}, \sigma_{i j}^{(\varepsilon)} \in W_{B} \mid\right.$ $\left.i, j \in \mathcal{O}^{k}\right\}, k=1, \ldots, l$, thus $W_{B}$ is a direct product $W^{1} \times \cdots \times W^{l}$ of groups acting on the direct sum $V_{B}=V^{1} \oplus \cdots \oplus V^{l}$ of spaces. The algebra $\mathcal{H}_{B}$ is then a tensor product

$$
\mathcal{H}_{B}=\left(S_{-\mathbf{1}}\left(V^{1}\right) \otimes \mathbb{C} W^{1} \otimes S_{-\mathbf{1}}\left(V^{1 *}\right)\right) \otimes \ldots \otimes\left(S_{-\mathbf{1}}\left(V^{l}\right) \otimes \mathbb{C} W^{l} \otimes S_{-\mathbf{1}}\left(V^{l *}\right)\right),
$$

of vector spaces, where $V^{k *}=\oplus\left\{\mathbb{C} \underline{y}_{i} \mid i \in \mathcal{O}^{k}\right\}$. Observe that each $S_{-\mathbf{1}}\left(V^{k}\right) \otimes \mathbb{C} W^{k} \otimes S_{-\mathbf{1}}\left(V^{k *}\right)$ is a subalgebra, because the braided commutator $C_{i j}$ of $\underline{y}_{j}$ and $x_{i}\left(i, j \in \mathcal{O}^{k}\right)$ may only contain generators $\sigma_{i j}^{(\varepsilon)}, t_{j}^{(\eta)}$ of $W_{B}$ that lie in $W^{k}$. Thus, $\underline{\mathcal{H}}\left(W^{k}\right):=S_{-\mathbf{1}}\left(V^{k}\right) \otimes \mathbb{C} W^{k} \otimes S_{-\mathbf{1}}\left(V^{k *}\right)$ is a braided Cherednik algebra.

Let us show that

$$
\mathcal{H}_{B}=\underline{\mathcal{H}}\left(W^{1}\right) \otimes \ldots \otimes \underline{\mathcal{H}}\left(W^{l}\right)
$$

is a braided tensor product of algebras in the category $\mathcal{M}_{\Gamma_{\mathbf{q}}, \mathcal{R}_{\mathbf{q}}}$. First of all, $V^{k}$ and $V^{* k}$ are $\Gamma_{\mathbf{q}}$-submodules of $V_{B}$ and $V_{B}^{*}$, respectively, because they are spanned by simultaneous eigenvectors of $\Gamma_{\mathbf{q}}$. Next, since $W^{k}$ is generated by its elements that appear with nonzero coefficients in braided commutators of $V^{k}$ and $V^{k *}$, and the braided commutator map is $\Gamma_{\mathbf{q}}$-equivariant (proved in Step 1 ), the group algebra $\mathbb{C} W^{k}$ is a $\Gamma_{\mathbf{q}}$-submodule of $\mathbb{C} W_{B}$. This gives the $\Gamma_{\mathbf{q}}$-structure on $\underline{\mathcal{H}}\left(W^{k}\right)$. It remains to check that the commutation relations between $\underline{\mathcal{H}}\left(W^{k}\right)$ and $\underline{\mathcal{H}}\left(W^{k^{\prime}}\right)$ inside $\mathcal{H}_{B}$ are the same as in the braided tensor product $\underline{\mathcal{H}}\left(W^{k}\right) \underline{\otimes} \underline{\mathcal{H}}\left(W^{k^{\prime}}\right)$.

In $\underline{\mathcal{H}}\left(W^{k}\right) \underline{\otimes} \underline{\mathcal{H}}\left(W^{k^{\prime}}\right)$, the variables $x_{i}$ and $x_{j}\left(i \in \mathcal{O}^{k}, j \in \mathcal{O}^{k^{\prime}}\right) q_{i j}$-commute, and the same happens in $\mathcal{H}_{B}$. Similarly for $\underline{y}_{i}$ and $\underline{y}_{j}$. Furthermore, $\underline{y}_{j}$ and $x_{i} q_{i j}$-commute in $\underline{\mathcal{H}}\left(W^{k}\right) \underline{\otimes} \underline{\mathcal{H}}\left(W^{k^{\prime}}\right)$, and the same happens in $\mathcal{H}_{B}$, since $C_{i j}=0: i, j$ are not linked, hence there is no element $\sigma_{i j}^{(\varepsilon)}$ in $W_{B}$. Finally, for any $w \in W^{k}$ and $w^{\prime} \in W^{k^{\prime}}$ one has $\mathcal{R}_{\mathbf{q}}\left(w\left(\gamma_{i}\right), w^{\prime}\left(\gamma_{j}\right)\right)=\mathcal{R}_{\mathbf{q}}\left(\gamma_{i}, \gamma_{j}\right)=-1$, simply because $w\left(\gamma_{i}\right) \in\left|V^{k}\right| \subset\left|V_{B}\right|, w^{\prime}\left(\gamma_{j}\right) \in\left|V^{k^{\prime}}\right| \subset\left|V_{B}\right|$, and the value of $\mathcal{R}_{\mathbf{q}}$ at any pair of distinct elements of $\left|V_{B}\right|$ is -1 as $B$ is a negative block. Hence by Proposition 4.6, $W^{k^{\prime}}$ commutes with $\underline{\mathcal{H}}\left(W^{k}\right)$ and $\underline{\mathcal{H}}\left(W^{k^{\prime}}\right)$ commutes with $W^{k}$ in $\underline{\mathcal{H}}\left(W^{k}\right) \underline{\otimes} \underline{\mathcal{H}}\left(W^{k^{\prime}}\right)$. But the same relations hold in $\mathcal{H}_{B}$, thus $\mathcal{H}_{B}=\underline{\mathcal{H}}\left(W^{1}\right) \underline{\otimes} \ldots \underline{\otimes} \underline{\mathcal{H}}\left(W^{l}\right)$.

Step 4: Proof that each of the algebras $\underline{\mathcal{H}}\left(W^{k}\right)$ is an "elementary" braided Cherednik algebra. Recall that we are working with a negative block $B$ of indices, and have already factorised $\mathcal{H}_{B}$ into braided Cherednik algebras $\underline{\mathcal{H}}\left(W^{k}\right), k=1, \ldots, l$, where $W^{k} \subset \mathrm{GL}\left(\mathcal{V}^{k}\right)$ and $V^{k}=\oplus\left\{\mathbb{C} x_{i} \mid\right.$ $\left.i \in \mathcal{O}^{k}\right\}$. We fix an index $k$ and will show that $\underline{\mathcal{H}}\left(W^{k}\right)$ is isomorphic to one of the "elementary" 
braided Cherednik algebras, listed in (1)-(4) before the Theorem. Without the loss of generality we may assume that the set $\mathcal{O}^{k}$ of indices is $1,2, \ldots, d$. If $d=1$, then $W^{k}$ is a cyclic group (an irreducible complex reflection group of rank 1), and $\underline{\mathcal{H}}\left(W^{k}\right)$ is a rational Cherednik algebra isomorphic to $H_{c}\left(W^{k}\right)$ or $H_{0, c}\left(W^{k}\right)$.

Assume $d \geq 2$. All indices in $\{1, \ldots, d\}$ are pairwise linked, that is for each pair $i, j \in\{1, \ldots, d\}$ of distinct indices, there is at least one nonzero number - call it $\varepsilon_{i j}$ - such that $\sigma_{i j}^{\left(\varepsilon_{i j}\right)} \in W^{k}$.

We may assume that $\varepsilon_{i j}=1$ for all $i \neq j$ in $\{1, \ldots, d\}$. Indeed, we may change the basis of $V^{k}$ by rescaling the variable $x_{i}$ by a factor of $\varepsilon_{12} \varepsilon_{23} \ldots \varepsilon_{i-1, i}$ and denote the new basis again by $\left\{x_{i}\right\}$. The braided Cherednik algebra structure of $\underline{\mathcal{H}}\left(W^{k}\right)$ obviously does not change under such rescaling, nor does the action of $\Gamma_{\mathbf{q}}$. We apply rescaling to the dual basis in $V^{k *}$ so that $\left\{x_{i}\right\}$ and $\left\{\underline{y}_{i}\right\}, i=1, \ldots, d$, remain a pair of dual bases. Now with respect to the new basis, $W^{k}$ contains $\sigma_{i-1, i}^{(1)}$ and hence also contains $\sigma_{i, i-1}^{(1)}=\left(\sigma_{i-1, i}^{(1)}\right)^{-1}$ for each $i=2, \ldots, d$. It then follows from the relation $\sigma_{b a}^{(1)} \sigma_{b c}^{(1)} \sigma_{a b}^{(1)}=\sigma_{a c}^{(1)}$ that $W^{k}$ contains $\sigma_{i j}^{(1)}$ for any pair $i \neq j, i, j=1, \ldots, d$.

Besides $\sigma_{i j}^{(1)}$, the group $W^{k}$ may have some other generators, namely some of $t_{j}^{(\eta)}$ and $\sigma_{i j}^{(\varepsilon)}$. We replace each generator $t_{j}^{(\eta)}$ by

$$
t_{1}^{(\eta)}=\sigma_{j 1}^{(1)} t_{j}^{(\eta)} \sigma_{1 j}^{(1)} \in W^{k}
$$

and each generator $\sigma_{i j}^{(\varepsilon)}$ by

$$
t_{1}^{(\varepsilon)} t_{2}^{\left(\varepsilon^{-1}\right)}=\sigma_{21}^{(1)} \sigma_{j 2}^{(1)} \sigma_{i 1}^{(1)} \sigma_{i j}^{(\varepsilon)} \sigma_{1 i}^{(1)} \sigma_{2 j}^{(1)} .
$$

Thus the new set of generators for the same group $W^{k}$ contains $\sigma_{i j}^{(1)}$ for all $i \neq j, i, j=1, \ldots, d$, and also $t_{1}^{(\eta)}$ and $t_{1}^{(\varepsilon)} t_{2}^{\left(\varepsilon^{-1}\right)}$ for some unknown choice of the $\eta$ 's and $\varepsilon^{\prime}$ 's. Let

$$
\mathcal{C}=\left\{\varepsilon \in \mathbb{C}^{\times} \mid t_{1}^{(\varepsilon)} t_{2}^{\left(\varepsilon^{-1}\right)} \in W^{k}\right\}, \quad \mathcal{C}^{\prime}=\left\{\eta \in \mathbb{C}^{\times} \mid t_{1}^{(\eta)} \in W^{k}\right\} .
$$

Then $\mathcal{C}$ (respectively $\mathcal{C}^{\prime}$ ) is a finite subgroup of $\mathbb{C}^{\times}$because it is the inverse image of $W^{k}$ under a group monomorphism $\varepsilon \mapsto t_{1}^{(\varepsilon)} t_{2}^{\left(\varepsilon^{-1}\right)}$ (respectively $\left.\eta \mapsto t_{1}^{(\eta)}\right)$ from $\mathbb{C}^{\times}$to $\operatorname{GL}\left(V^{k}\right)$. Moreover, $\mathcal{C}^{\prime} \subseteq \mathcal{C}$ because if $t_{1}^{(\eta)} \in W^{k}$, then $t_{1}^{(\eta)} t_{2}^{\left(\eta^{-1}\right)}=t_{1}^{(\eta)}\left[\sigma_{12}^{(1)} t_{1}^{(\eta)} \sigma_{21}^{(1)}\right]^{-1}$ is also in $W^{k}$. Besides that, $\mathcal{C}$ contains -1 , as $t_{1}^{(-1)} t_{2}^{(-1)}=\left(\sigma_{12}^{(1)}\right)^{2} \in W^{k}$, hence $\mathcal{C}$ is of even order. We have proved that

$$
W^{k}=W_{\mathcal{C}, \mathcal{C}^{\prime}}(d)
$$

By Proposition 3.10, $\underline{\mathcal{H}}\left(W^{k}\right)$ is isomorphic to $\underline{\mathcal{H}}_{c}\left(W_{\mathcal{C}, \mathcal{C}^{\prime}}(d)\right)$ or to $\underline{\mathcal{H}}_{0, c}\left(W_{\mathcal{C}, \mathcal{C}^{\prime}}(d)\right)$ for some parameter $c$.

Remark 4.10. Note that to form a braided tensor product $\underline{\mathcal{H}}=\underline{\mathcal{H}}_{1} \underline{\otimes} \ldots \underline{\otimes} \underline{\mathcal{H}} \underline{m}_{m}$ of braided Cherednik algebras of irreducible groups (algebras listed in (1)-(4) above), one needs $m(m-1) / 2$ extra nonzero complex parameters $r_{a b}, 1 \leq a<b \leq m$. The matrix $\mathbf{q}$ for $\underline{\mathcal{H}}$ can be written as a block matrix with $m^{2}$ blocks $M_{a b}, a, b=1, \ldots, m$, such that: 
- the size of $M_{a b}$ is $\left(\operatorname{rank} \underline{\mathcal{H}}_{a} \times \operatorname{rank} \underline{\mathcal{H}}_{b}\right)$;

- in a diagonal block $M_{a a}$, all entries are 1 or else all entries outside the main diagonal are -1 ;

- in an off-diagonal block $M_{a b}$ where $a<b$ (respectively $a>b$ ), all entries are equal to $r_{a b}$ (respectively $r_{a b}^{-1}$ ).

The commutation relations in the braided tensor product include $x x^{\prime}=r_{a b} x^{\prime} x$ whenever $x$ is one of the $x_{i}$ variables in $\underline{\mathcal{H}}_{a}$ and $x^{\prime}$ is one of the $x_{i}$ variables in $\underline{\mathcal{H}}_{b}$.

\section{Universal embeddings and BRAided Dunkl operators}

In the last Section of the paper, we embed braided Cherednik algebras in modified Heisenberg quadratic doubles, introduced here. We use this result to arrive at the explicit formulae for braided Dunkl operators.

5.1. Degenerate q-Cherednik algebras and Heisenberg quadratic doubles. We say that a q-Cherednik algebra of the form $\mathcal{H}_{0, c}(W)=S_{\mathbf{q}}(V) \otimes \mathbb{C} W \otimes S_{\mathbf{q}^{\top}}\left(V^{*}\right)$ is degenerate, if the commutator of $y \in V^{*}$ and $x \in V$ in $\mathcal{H}_{0, c}(W)$ is $\beta^{\prime}(y \otimes x)=y x-x y=\sum_{B} \gamma_{B} \sum_{s} c_{s}\left\langle x, \alpha_{s}^{\vee}\right\rangle\left\langle\alpha_{s}, y\right\rangle s$ (compare with Theorem 2.14). Here and below $B$ are blocks of indices with respect to the matrix $\mathbf{q}$, and $s$ runs over complex reflections in $\mathrm{GL}\left(V_{B}\right) \subset \mathrm{GL}(V)$; we continue to use the notation introduced in Section 2. We would like to construct a morphism (not necessarily injective) from a degenerate q-Cherednik algebra to a Heisenberg quadratic double over $W$. This is done via Theorem 1.21, the crucial step is to represent $\mathcal{H}_{0, c}(W)$ as a $\star$-product (Definition 1.14) of two quadratic doubles. This is done as follows:

$$
\mathcal{H}_{0, c}(W)=\mathcal{H}_{0, c}(W) \star \mathcal{H}_{0, c_{0}}(W)
$$

where the value of the fixed parameter $c_{0}$ at $s$ is $\left\langle\alpha_{s}, \alpha_{s}^{\vee}\right\rangle^{-1}$. Application of Theorem 1.21 now yields a Yetter-Drinfeld module

$$
Y_{\mathbf{q}}=\bigoplus_{B, s} \mathbb{C} \cdot\left[\gamma_{B} s\right]
$$

We refer to elements $\gamma_{B} s \in W$ as q-reflections. The YD module structure on $Y_{\mathbf{q}}$ is induced by its embedding in the YD module $\mathbb{C} W \otimes V$ via $\left[\gamma_{B} s\right]=\gamma_{B} s \otimes \alpha_{s}$. The dual YD module $Y_{\mathbf{q}}^{*}=$ $\bigoplus_{B, s} \mathbb{C} \cdot\left[\gamma_{B} s\right]^{*}$ embeds in $\mathbb{C} W \otimes V^{*}$ via $\left[\gamma_{B} s\right]^{*}=\left(\gamma_{B} s\right)^{-1} \otimes\left\langle\alpha_{s}, \alpha_{s}^{\vee}\right\rangle^{-1} \alpha_{s}^{\vee}$. By Theorem 1.21] the $W$-equivariant maps $\mu_{c}: V \rightarrow Y_{\mathbf{q}}, \nu: V^{*} \rightarrow Y_{\mathbf{q}}^{*}$ given by

$$
\mu_{c}(x)=\sum_{B, s} c_{s}\left\langle x, \alpha_{s}^{\vee}\right\rangle\left[\gamma_{B} s\right], \quad \nu(y)=\sum_{B, s}\left\langle\alpha_{s}, y\right\rangle\left[\gamma_{B} s\right]^{*}
$$

extend to an algebra morphism $\mathcal{H}_{0, c}(W) \rightarrow A_{Y_{\mathbf{q}}}=S\left(Y_{\mathbf{q}}, \Psi_{Y_{\mathbf{q}}}\right) \otimes \mathbb{C W} \otimes S\left(Y_{\mathbf{q}}^{*}, \Psi_{Y_{\mathbf{q}}}^{*}\right)$. 
5.2. q-Cherednik algebras are subalgebras in modified Heisenberg quadratic doubles. One can obtain a version of the above morphism $\mathcal{H}_{0, c}(W) \rightarrow A_{Y_{\mathbf{q}}}$ for non-degenerate q-Cherednik algebras. A new ingredient for this is the operation $\diamond$, introduced in Section 1 ,

Suppose that $Y$ is a module over a group $W$, and that $Y$ has two different Yetter-Drinfeld structures over $W$; that is, two $W$-gradings satisfying the Yetter-Drinfeld axiom with respect to the same $W$-action on $Y$. These two YD structures give rise to two braidings $\Psi_{1}, \Psi_{2}$ on $Y$ and two Heisenberg quadratic doubles

$$
A_{Y}^{i}=S\left(Y, \Psi_{i}\right) \otimes \mathbb{C} W \otimes S\left(Y^{*}, \Psi_{i}^{*}\right), \quad i=1,2 .
$$

Definition 5.1. The quadratic double $A_{Y}^{1} \diamond A_{Y}^{2}$ is called a modified Heisenberg quadratic double of the two Yetter-Drinfeld structures on $Y$.

By definition of $\diamond$, the triangular decomposition of $A_{Y}^{1} \diamond A_{Y}^{2}$ is

$$
\frac{T(Y)}{<\operatorname{ker}\left(\mathrm{id}+\Psi_{1}\right) \cap \operatorname{ker}\left(\mathrm{id}+\Psi_{2}\right)>} \otimes \mathbb{C} W \otimes \frac{T\left(Y^{*}\right)}{<\operatorname{ker}\left(\mathrm{id}+\Psi_{1}^{*}\right) \cap \operatorname{ker}\left(\mathrm{id}+\Psi_{2}^{*}\right)>} .
$$

Now, by Theorem 2.14 an arbitrary q-Cherednik algebra can be written as $S_{\mathbf{q}}(V) \otimes \mathbb{C} W \otimes S_{\mathbf{q}^{\top}}\left(V^{*}\right)$ with the commutator $\beta(y \otimes x)=\sum_{B} \gamma_{B}(x, y)_{B}+\beta^{\prime}(y \otimes x)$ between $V^{*}$ and $V$, with $\beta^{\prime}(y \otimes x)$ as above. Denote this algebra by $\mathcal{H}_{(\cdot, \cdot), c}(W)$, where $(\cdot, \cdot)=\sum_{B} \gamma_{B}(\cdot, \cdot)_{B}$ is the $\mathbb{C} \bar{\Gamma}$-valued pairing between $V$ and $V^{*}$. Clearly,

$$
\mathcal{H}_{(\cdot, \cdot), c}(W)=\mathcal{H}_{0, c}(W) \diamond \mathcal{H}_{(\cdot, \cdot), 0}(W),
$$

where $\mathcal{H}_{0, c}(W)$ is the degenerate $\mathbf{q}$-Cherednik algebra with commutator $\beta^{\prime}$ as above, and $\mathcal{H}_{(\cdot, \cdot), 0}(W)$ is the $\mathbf{q}$-Cherednik algebra with commutator $\beta-\beta^{\prime}$. We have already constructed a morphism $\mathcal{H}_{0, c}(W) \rightarrow A_{Y_{\mathbf{q}}}$, and will now turn to the algebra $\mathcal{H}_{(\cdot, \cdot), 0}(W)$.

Recall that the module $Y_{\mathbf{q}}$ has $W$-grading given by

$$
\left|\left[\gamma_{B} s\right]\right|=\gamma_{B} s .
$$

Assume that the group $W$ contains the Abelian group

$$
\bar{\Gamma}=\left\langle\gamma_{B_{1}}, \ldots, \gamma_{B_{N}}\right\rangle \subset \mathrm{GL}(V) ;
$$

since $W$ permutes subspaces $V_{B}$, the group $\bar{\Gamma}$ is normal in $W$. It follows that we can introduce the second, $\bar{\Gamma}$-valued grading

$$
\left|\left[\gamma_{B} s\right]\right|_{\bar{\Gamma}}:=\gamma_{B}
$$

on $Y_{\mathbf{q}}$, which also makes $Y_{\mathbf{q}}$ a Yetter-Drinfeld module over $W$. This second YD structure leads to a Heisenberg quadratic double

$$
A_{Y_{\mathbf{q}}}^{\overline{\bar{\Gamma}}} \cong S\left(Y_{\mathbf{q}}, \tau_{\bar{\Gamma}}\right) \otimes \mathbb{C} W \otimes S\left(Y_{\mathbf{q}}^{*}, \tau_{\bar{\Gamma}}^{*}\right)
$$


where the braiding $\tau_{\bar{\Gamma}}$ is given by $\tau_{\bar{\Gamma}}\left(\left[\gamma_{B} s\right] \otimes\left[\gamma_{C} t\right]\right)=q_{B, C}\left[\gamma_{C} t\right] \otimes\left[\gamma_{B} s\right]$. Clearly, $S\left(Y_{\mathbf{q}}, \tau_{\bar{\Gamma}}^{*}\right)$ is nothing but a $\left(q_{B, C}\right)$-polynomial algebra of $Y_{\mathbf{q}}$.

Lemma 5.2. For some pairings $(\cdot, \cdot)_{B}$, the maps $\mu_{c}: V \rightarrow Y_{\mathbf{q}}, \nu: V^{*} \rightarrow Y_{\mathbf{q}}^{*}$ defined above extend to a morphism $\mathcal{H}_{(\cdot, \cdot), 0}(W) \rightarrow A_{Y_{\mathbf{q}}}^{\bar{\Gamma}}$. This morphism is injective if the roots $\left\{\alpha_{s} \mid c_{s} \neq 0\right\}$ span $V$.

Proof. Let $B, C \subset\{1, \ldots, n\}$ be blocks of indices. For any $i \in B, j \in C, i \neq j$ the relation $x_{i} x_{j}=q_{B, C} x_{j} x_{i}$ holds in $S_{\mathbf{q}}(V)$. Note that $\mu_{c}\left(x_{i}\right)$ is a linear combination of $\left[\gamma_{B} s\right]$ where $s \in W$ runs over complex reflections in $V_{B}$, and similarly $\mu_{c}\left(x_{j}\right)$ is a combination of [ $\gamma_{C} t$ ] where $t$ runs over complex reflections in $V_{C}$. The relation $\left[\gamma_{B} s\right]\left[\gamma_{C} t\right]=q_{B, C}\left[\gamma_{C} t\right]\left[\gamma_{B} s\right]$ holds in $S\left(Y_{\mathbf{q}}, \tau_{\bar{\Gamma}}\right)$ for any such $s, t$, hence $\mu_{c}$ extends to a morphism $S_{\mathbf{q}}(V) \rightarrow S\left(Y_{\mathbf{q}}, \tau_{\bar{\Gamma}}\right)$. If $\left\{\alpha_{s} \mid c_{s} \neq 0\right\}$ span $V, \mu_{c}: V \rightarrow Y_{\mathbf{q}}$ is injective; then $\mu_{c}\left(x_{i}\right)$ are linearly independent vectors in $Y_{\mathbf{q}}$ that generate a subalgebra in $S\left(Y_{\mathbf{q}}, \tau_{\bar{\Gamma}}\right)$ isomorphic to the $\mathbf{q}$-polynomial algebra, therefore the extension of $\mu_{c}$ to $S_{\mathbf{q}}(V)$ is injective. Similarly, $\nu$ extends to a morphism $S_{\mathbf{q}^{\top}}\left(V^{*}\right) \rightarrow S\left(Y_{\mathbf{q}}^{*}, \tau_{\bar{\Gamma}}^{*}\right)$, injective if all $\alpha_{s}^{\vee}$ span $V^{*}$ (that is, all $\alpha_{s}$ span $\left.V\right)$.

The $\mathbb{C} \bar{\Gamma}$-valued commutator of $\nu\left(y_{j}\right)$ and $\mu_{c}\left(x_{i}\right)$ in $A_{Y_{\mathbf{q}}}^{\overline{\Gamma_{n}}}$ is equal to $\delta_{B, C} \gamma_{B} \sum_{s} c_{s}\left\langle x, \alpha_{s}^{\vee}\right\rangle\left\langle\alpha_{s}, y\right\rangle$ where $s \in W$ runs over complex reflections in $V_{B}$. Let the same formula define the pairing $\gamma_{B}\left(x_{i}, y_{j}\right)_{B}$ between $V$ and $V^{*}$. Then we have a morphism $\mathcal{H}_{(\cdot, \cdot), 0}(W) \rightarrow A_{Y_{\mathbf{q}}}^{\bar{\Gamma}}$ that extends the maps $\mu_{c}, \nu$. It follows from the triangular decomposition that if this morphism is injective on $S_{\mathbf{q}}(V)$ and $S_{\mathbf{q}^{\top}}\left(V^{*}\right)$, it is injective on $\mathcal{H}_{(\cdot, \cdot), 0}(W)$.

Applying Proposition 1.18, we obtain

Theorem 5.3. If the parameter $c$ is such that the roots $\left\{\alpha_{s} \mid c_{s} \neq 0\right\}$ span $V$, then for some pairings $(\cdot, \cdot)_{B}$, the above maps $\mu_{c}: V \rightarrow Y_{\mathbf{q}}, \nu: V^{*} \rightarrow Y_{\mathbf{q}}^{*}$ extend to an embedding $\mathcal{H}_{(\cdot, \cdot), c}(W) \hookrightarrow$ $A_{Y_{\mathbf{q}}} \diamond A_{Y_{\mathbf{q}}}^{\bar{\Gamma}}$.

Remark 5.4. The $W$-invariant $\mathbb{C} \bar{\Gamma}$-valued pairing $(\cdot, \cdot)$ between $V$ and $V^{*}$ in the Theorem depends on the parameter $c$. As in any q-Cherednik algebra, this pairing is of a special kind: namely, it is obtained from a scalar $W$-invariant pairing by the change of variables as in Corollary 3.7 . If the group $W$ is irreducible, such pairing is unique up to a scalar factor. One deduces that any q-Cherednik algebra of an irreducible group, with at least one nonzero parameter $c_{s}$, embeds in a modified Heisenberg double.

The following is left as an exercise to the reader:

Exercise. Describe an embedding of the twist of a rational Cherednik algebra, introduced just before Corollary [3.7, in an appropriate version of a modified Heisenberg double. 
Remark 5.5. Embedding of a braided Cherednik algebra $\underline{\mathcal{H}}(W)$ in a modified braided Heisenberg double, described in Theorem 0.9 , is obtained by first embedding $\underline{\mathcal{H}}(W)$ in a q-Cherednik algebra $\mathcal{H}(\widetilde{W})$ with $\widetilde{W}=W \cdot \Gamma_{\mathbf{q}} \cdot \bar{\Gamma}$, then applying Theorem 5.3 to $\mathcal{H}(\widetilde{W})$.

5.3. Braided Dunkl operators. We will now consider the braided Cherednik algebra $\underline{\mathcal{H}}_{c}(W)$ of the irreducible group $\left.W=W_{\mathcal{C}, \mathcal{C}^{\prime}}\right)$, as introduced in Definition 3.5 The parameter $c$ is a function $c: \mathcal{C}^{\prime} \rightarrow \mathbb{C}$ (with the exception of rank $n=2$, see Remark 3.9] we are going to ignore this exception and claim that in rank 2, the proof may easily be modified as appropriate). The algebra has triangular decomposition $\underline{\mathcal{H}}_{c}(W)=S_{-\mathbf{1}}(V) \otimes \mathbb{C} W \otimes S_{-\mathbf{1}}\left(V^{*}\right)$ where $V$ is spanned by $x_{1}, \ldots, x_{n}$ and $V^{*}$ is spanned by $\underline{y}_{1}, \ldots, \underline{y}_{n}$. The group $\Gamma_{-\mathbf{1}}$ is generated by $n$ commuting involutions $\gamma_{i}, \gamma_{i}\left(x_{j}\right)=-1$ $(i \neq j), \gamma_{i}\left(x_{i}\right)=1$.

Denote by $p r$ the projection $\operatorname{id}_{S_{-1}(V)} \otimes \varepsilon_{W} \otimes \varepsilon_{V^{*}}: S_{-\mathbf{1}}(V) \otimes \mathbb{C} W \otimes S_{-\mathbf{1}}\left(V^{*}\right) \rightarrow S_{-\mathbf{1}}(V)$ onto $S_{-1}(V)$, where $\varepsilon_{W}: \mathbb{C} W \rightarrow \mathbb{C}$ is the algebra morphism such that $\varepsilon_{W}(w)=1$ for $w \in W$, and $\varepsilon_{V^{*}}: S_{-\mathbf{1}}\left(V^{*}\right) \rightarrow \mathbb{C}$ is the algebra morphism such that $\varepsilon_{V^{*}}\left(V^{*}\right)=0$. The braided Dunkl operators attached to the group $W$ are

$$
\underline{\nabla}_{i}: S_{-\mathbf{1}}(V) \rightarrow S_{-\mathbf{1}}(V), \quad \underline{\nabla}_{i}(a)=\operatorname{pr}\left(\underline{y}_{i} a\right) .
$$

Our last goal is to prove formula (11) for $\underline{\nabla}_{i}$, given in the Introduction.

We put $\widetilde{W}=W \cdot \Gamma_{-\mathbf{1}} \cdot\{ \pm \mathrm{id}\}$ and embed $\underline{\mathcal{H}}_{c}(W)$, as a braided reduction, in the q-Cherednik algebra $\mathcal{H}_{c}(\widetilde{W})$. Explicitly, we have $\underline{y}_{i}=\gamma_{i}^{-1} y_{i}=\gamma_{i} y_{i}$ for $i=1, \ldots, n$, where $y_{1}, \ldots, y_{n}$ span $V^{*}$ in $\mathcal{H}_{c}(\widetilde{W})$. The q-complex reflections in $\widetilde{W}$ are

$$
-\mathrm{id} \cdot s_{i j}^{(\varepsilon)}, \varepsilon \in \mathcal{C} ; \quad-\mathrm{id} \cdot t_{i}^{\left(\varepsilon^{\prime}\right)}, \varepsilon^{\prime} \in \pm \mathcal{C}^{\prime}
$$

where $-\mathrm{id} \cdot s_{i j}^{(\varepsilon)}=\gamma_{i} \sigma_{i j}^{(\varepsilon)}$. (Formally, the parameters $c$ in $\underline{\mathcal{H}}_{c}(W)$ and in $\mathcal{H}_{c}(\widetilde{W})$ are not the same, but they are identified in a rather obvious way.) We can find the generalised Dunkl operators $\nabla_{i}$ for the algebra $\mathcal{H}(\widetilde{W})$ and then put $\underline{\nabla}_{i}=\gamma_{i} \nabla_{i}$.

The generalised Dunkl operators $\nabla_{i}$ will be computed using the procedure described in 1.6. First, we embed $\mathcal{H}_{c}(\widetilde{W})$ in a modified Heisenberg quadratic double as in 5.2 . We then have the following "generalised root system" of 1.6

$$
\alpha_{-\mathrm{id} \cdot s_{i j}^{(\varepsilon)}}=x_{i}-\varepsilon x_{j}, \quad \alpha_{-\mathrm{id} \cdot s_{i j}^{(\varepsilon)}}^{\vee}=c_{1}\left(y_{i}-\varepsilon^{-1} y_{j}\right), \quad \alpha_{-\mathrm{id} \cdot t_{i}^{\left(\varepsilon^{\prime}\right)}}=x_{i}, \quad \alpha_{-\mathrm{id} \cdot t_{i}^{\left(\varepsilon^{\prime}\right)}}^{\vee}=c_{\varepsilon^{\prime}}\left(1-\varepsilon^{\prime}\right) y_{i} .
$$

It follows from 1.6 that

$$
\nabla_{i}=\partial_{i}+\sum_{w}\left\langle\alpha_{w}, y_{i}\right\rangle \bar{\partial}_{w}
$$


where $\partial_{i}$ is defined by $\partial_{i}\left(x_{1}^{a_{1}} \ldots x_{n}^{a_{n}}\right)=a_{i} x_{1}^{a_{1}} \ldots x_{i}^{a_{i}-1} \ldots x_{n}^{a_{n}}$ and $\bar{\partial}_{w}$ are uniquely defined operators on $S_{-1}(V)$ satisfying

$$
\bar{\partial}_{w}(x)=\left\langle x, \alpha_{w}^{\vee}\right\rangle, \quad \bar{\partial}_{w}(a b)=\bar{\partial}_{w}(a) w(b)+a \bar{\partial}_{w}(b),
$$

for $x \in V, a, b \in S_{-\mathbf{1}}(V)$. If we know that $\bar{\partial}_{w}$ lowers the degree in $S_{-\mathbf{1}}(V)$ by 1 , both rules are equivalent to the equation

$$
\left[\bar{\partial}_{w}, x\right]=\left\langle x, \alpha_{w}^{\vee}\right\rangle w
$$

$\operatorname{in} \operatorname{End}_{\mathbb{C}}\left(S_{-\mathbf{1}}(V)\right)$. Put $\sigma_{i j}:=\sigma_{i j}^{(1)}$ and define

$$
D_{i j}=\frac{1}{x_{i}^{2}-x_{j}^{2}}\left(\left(x_{i}+x_{j}\right)\left(1-\sigma_{i j}\right)+\left(x_{i}-x_{j}\right)\left(1-\sigma_{j i}\right)\right) .
$$

We claim that $c_{1} \gamma_{i} D_{i j}=\bar{\partial}_{-\mathrm{id} \cdot s_{i j}^{(1)}}+\bar{\partial}_{-\mathrm{id} \cdot s_{i j}^{(-1)}}$. Indeed, it is not difficult to check, using the anticommutativity of the $x_{i}$ and the fact that $x_{i}^{2}-x_{j}^{2}$ is central in $S_{-\mathbf{1}}(V)$, that

$\left[\gamma_{i} D_{i j}, x_{i}\right]=(-\mathrm{id}) \cdot s_{i j}^{(1)}+(-\mathrm{id}) \cdot s_{i j}^{(-1)}, \quad\left[\gamma_{i} D_{i j}, x_{j}\right]=(-\mathrm{id}) \cdot s_{i j}^{(1)}-(-\mathrm{id}) \cdot s_{i j}^{(-1)}, \quad\left[\gamma_{i} D_{i j}, x_{k}\right]=0$.

Conjugating evertyhing with $t_{j}^{(\varepsilon)}$ shows that $c_{1} \gamma_{i} t_{j}^{(\varepsilon)} D_{i j} t_{j}^{(\varepsilon)-1}=\bar{\partial}_{-\mathrm{id} \cdot s_{i j}^{(\varepsilon)}}+\bar{\partial}_{-\mathrm{id} \cdot s_{i j}^{(-\varepsilon)}}$.

In the same way it is shown that if $D_{i}^{\left(\varepsilon^{\prime}\right)}=\frac{1}{x_{i}}\left(1-t_{i}^{\left(\varepsilon^{\prime}\right)}\right)$, then $c_{\varepsilon^{\prime}} \gamma_{i} D_{i}=\bar{\partial}_{t_{i}^{\left(\varepsilon^{\prime}\right)}}$ because

$$
\left[\gamma_{i} D_{i}^{\left(\varepsilon^{\prime}\right)}, x_{k}\right]=\delta_{i k}\left(1-\varepsilon^{\prime}\right) t_{i}^{\left(\varepsilon^{\prime}\right)}
$$

(verified directly). We thus have the following expansion for $\nabla_{i}$ :

$$
\nabla_{i}=\partial_{i}+\gamma_{i} c_{1} \sum_{j \neq i, \varepsilon \in \widetilde{\mathcal{C}}} t_{j}^{(\varepsilon)} D_{i j} t_{j}^{(\varepsilon)-1}+\gamma_{i} \sum_{\varepsilon^{\prime} \in \mathcal{C}^{\prime} \backslash\{1\}} \frac{c_{\varepsilon^{\prime}}}{1-\varepsilon^{\prime}} D_{i}^{\left(\varepsilon^{\prime}\right)}
$$

where $\widetilde{\mathcal{C}}$ is a set of $|\mathcal{C}| / 2$ elements of $\mathcal{C}$ distinct modulo the subgroup $\{ \pm 1\}$. Multiplying by $\gamma_{i}$ on the left and observing that $\gamma_{i} \partial_{i}=\underline{\partial_{i}}$, we obtain formula (10) for $\underline{\nabla}_{i}$ as given in the Introduction.

\section{REFERENCES}

[AS1] N. Andruskiewitsch, H.-J. Schneider, Finite Quantum Groups and Cartan Matrices, Adv. Math. 154 (2000), $1-45$.

[AS2] N. Andruskiewitsch, H.-J. Schneider, Pointed Hopf algebras, in: New directions in Hopf algebras, MSRI Publ., 43, Cambridge Univ. Press, Cambridge, 2002, 1-68.

[BEER] L. Bartholdi, B. Enriquez, P. Etingof, E. Rains. Groups and Lie algebras corresponding to the Yang-Baxter equations, J. Algebra 305 (2006), no. 2, 742-764.

[B] Y. Bazlov, Nichols-Woronowicz algebra model for Schubert calculus on Coxeter groups, J. Algebra 297 (2006), no. 2, 372-399.

[BB] Y. Bazlov, A. Berenstein, Braided doubles and rational Cherednik algebras, preprint arXiv:0706.0243

[BB3] Y. Bazlov, A. Berenstein, Noncommutative tori and extended braided Cherednik algebras, in preparation.

[BK] Y. Bazlov, A. Kirillov, Deformed Demazure operators and classical Yang-Baxter algebras, in preparation. 
[BEG] Y. Berest, P. Etingof, V. Ginzburg, Finite-dimensional representations of rational Cherednik algebras. Int. Math. Res. Not. 2003, no. 19, 1053-1088.

[Dr] V. Drinfeld, Degenerate affine Hecke algebras and Yangians. Funktsional. Anal. i Prilozhen. 20 (1986), no. $1,69-70$

[D] C. F. Dunkl, Differential-difference operators associated to reflection groups, Trans. AMS 311 (1989), no. 1, $167-183$.

[DO] C. F. Dunkl, E. M. Opdam, Dunkl operators for complex reflection groups. Proc. London Math. Soc. (3) 86 (2003), no. 1, 70-108.

[EG] P. Etingof, V. Ginzburg, Symplectic reflection algebras, Calogero-Moser space, and deformed Harish-Chandra homomorphism. Invent. Math. 147 (2002), no. 2, 243-348.

[FK] S. Fomin, A. N. Kirillov, Quadratic algebras, Dunkl elements, and Schubert calculus. Advances in geometry, 147-182, Progr. Math., 172, Birkhäuser Boston, Boston, MA, 1999.

[G] I. Gordon, Baby Verma modules for rational Cherednik algebras. Bull. London Math. Soc. 35 (2003), no. 3, $321-336$.

[KW1] T. Khongsap, W. Wang, Hecke-Clifford algebras and spin Hecke algebras I: the classical affine type, to appear in Transformation Groups, preprint arXiv:0704.0201

[KW2] T. Khongsap, W. Wang, Hecke-Clifford algebras and spin Hecke algebras II: the rational double affine type, preprint arXiv:0710.5877

$[\mathrm{KM}]$ A.N. Kirillov, T. Maeno, Exterior differential algebras and flat connections on Weyl groups, Proc. Japan Acad. Ser. A Math. Sci. 81 (2005), no. 2, 30-35.

[M1] S. Majid, Free braided differential calculus, braided binomial theorem, and the braided exponential map. $J$. Math. Phys. 34 (1993), no. 10, 4843-4856.

[M2] S. Majid, A Quantum Groups Primer. London Mathematical Society Lecture Note Series 292, Cambridge University Press, 2002.

[M3] S. Majid, Noncommutative differentials and Yang-Mills on permutation groups $S_{N}$, Lect. Notes Pure Appl. Maths, 239, Marcel Dekker, 2004, pp. 189-214.

[N] W.D. Nichols, Bialgebras of type one, Comm. Algebra 6 (15) (1978), 1521-1552.

[ST] G. C. Shephard, J. A. Todd, Finite unitary reflection groups. Canadian J. Math. 6 (1954), 274-304.

[VV] M. Varagnolo, E. Vasserot, Finite dimensional representations of DAHA and affine Springers fibers: the spherical case, preprint arXiv:0705.2691

[W] S. L. Woronowicz, Differential calculus on compact matrix pseudogroups (quantum groups). Comm. Math. Phys. 122 (1989), no. 1, 125-170.

Mathematics Institute, University of Warwick, Coventry CV4 7AL, UK

E-mail address: y.bazlov@warwick.ac.uk

Department of Mathematics, University of Oregon, Eugene, OR 97403, USA

E-mail address: arkadiy@math.uoregon.edu 